Operaaores em subespaços de

Espaços de Banach

Hereditariamente Indecomponíveis

\author{
Robson Rodrigues da Silva \\ DISSERTAÇÃO APRESENTADA \\ $\mathrm{AO}$
}

INSTITUTO DE MATEMÁTICA E ESTATÍSTICA

DA

UNIVERSIDADE DE SÃO PAULO

PARA

OBTENÇÃO DO GRAU

$\mathrm{DE}$

MESTRE EM MATEMÁTICA

Área de Concentração: Análise

Orientador: Prof. Dr. Elói Medina Galego

São Paulo - Outubro - 2004 


\title{
Operadores em subespaços de Espaços de Banach
} Hereditariamente Indecomponíveis

\author{
Este exemplar corresponde à redação \\ final da dissertação devidamente \\ corrigida e defendida por \\ Robson Rodrigues da Silva \\ e aprovada pela comissão julgadora.
}

São Paulo, outubro de 2004

Banca examinadora:

- Prof. Dr. Elói Medina Galego (Orientador) - IME - USP

- Prof. Dr. Chaim Samuel Hönig - IME - USP

- Prof. Dr. Valentin Ferenczi - PARIS VI 
" A matemática não é uma caminhada cuidadosa através de uma estrada bem conhecida, é uma jornada por uma terra selvagem e estranha, onde os exploradores freqüentemente se perdem. A exatidão deve ser um sinal aos historiadores de que os mapas já foram feitos e os exploradores se foram para outras terras."

W.S. Anglin 


\section{AGRADECIMENTOS}

Ao Prof. Elói Medina Galego, não só pela sua paciente orientação, como também pelo incentivo e força nos momentos em que mais precisei.

$>$ A minha esposa Edna e minha filha Camila pela compreensão, paciência e amor durante todos os momentos em que estava realizando este trabalho.

Aos meus pais.

$>$ Aos meus irmãos pelas horas dispensadas aos meus estudos.

Ao Prof. Valentin Ferenczi pelas dúvidas esclarecidas por vários e-mails.

A Prof. Iracema Martin Bund por ter me colocado no caminho certo e no momento certo, meus profundos e sinceros agradecimentos.

$>$ A todos aqueles que direta ou indiretamente contribuíram para a realização deste trabalho.

Ao Senhor, por ter me dado paz e saúde para trilhar essa caminhada. 


\section{RESUMO}

O objetivo desse trabalho é apresentar em detalhes a demonstração de um resultado fundamental sobre "Operadores em subespaços de espaços de Banach hereditariamente indecomponíveis".

Em 1993, W. T. Gowers e B. Maurey mostraram que sendo X um espaço de Banach complexo hereditariamente indecomponível, todo operador de $X$ em $X$ é da forma $\lambda I_{X}+S$, onde $\mathrm{I}_{\mathrm{x}}$ é a identidade, $\mathrm{S}$ um operador estritamente singular e $\lambda$ um número complexo. Nós apresentaremos uma generalização deste resultado, mostrando que para todo subespaço fechado $Y$ de $X$, todo operador de $Y$ em $X$ é da forma $\lambda I_{Y}+S$. Esse último resultado foi provado por V. Ferenczi e publicado em Bull. London Math. Soc. 29 (1996) 338 - 344.

\section{ABSTRACT}

The purpose of this work is to present in details, a demonstration of a fundamental result about "Operators on subspaces of hereditarily indecomposable Banach spaces".

In 1993, W. T. Gowers e B. Maurey showed that if $X$ is a complex Banach space hereditarily indecomposable, then every operator from $X$ to $X$ is the form $\lambda I_{X}+S$, where $I_{x}$ is the identity map, $S$ is strictly singular operator and $\lambda$ is a complex number. We present a generalization of this result, showing that for every closed subspace $Y$ of $X$, every operator from $Y$ to $X$ is the form $\lambda I_{Y}+S$. It was proved by V. Ferenczi in Bull. London Math. Soc. 29 (1996) $338-344$. 


\section{SUMÁRIO}

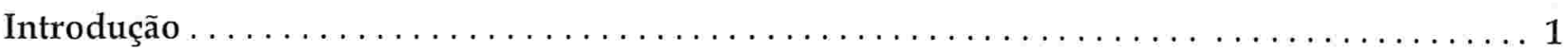

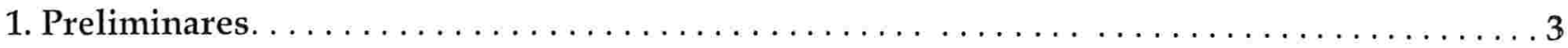

1.1 Notações. . . . . . . . . . . . . . . . . . . . . . . . . . . . . . . .

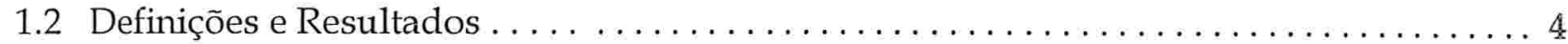

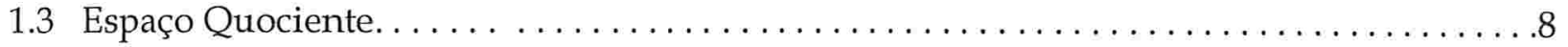

1.4 Espaços de Banach Hereditariamente Indecomponíveis . . . . . . . . . . . . . . . 10

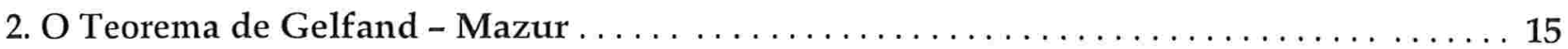

2.1 Álgebras Normadas . . . . . . . . . . . . . . . . . . . . . . . . . 15

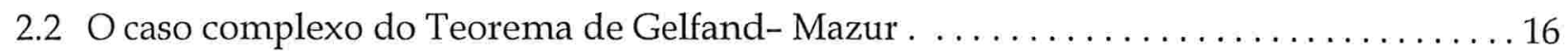

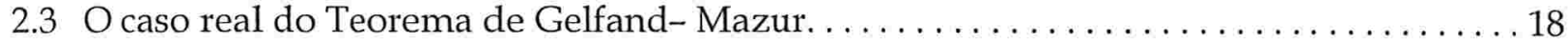

3. Operadores Estritamente Singulares. Operadores Compactos. Operadores de Fredholm . . 26

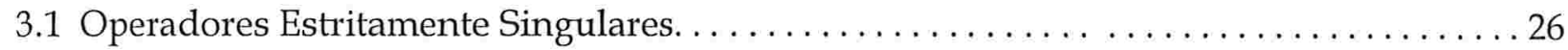

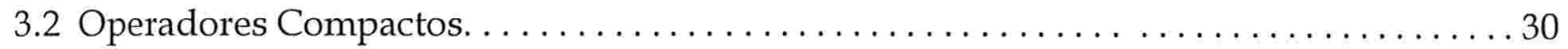

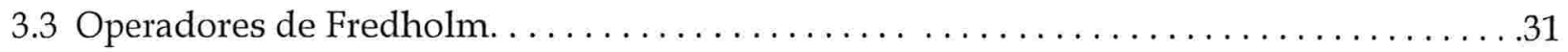

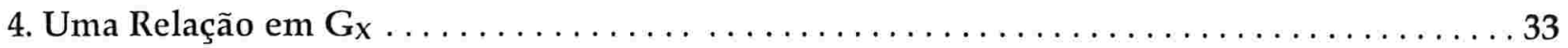

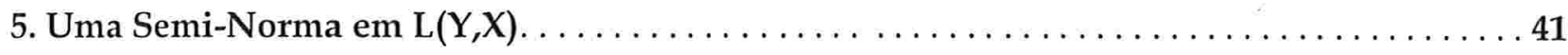

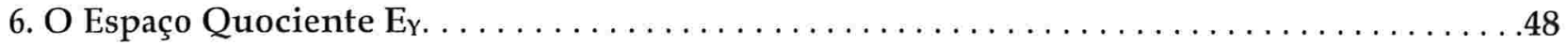

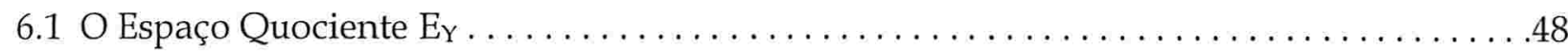

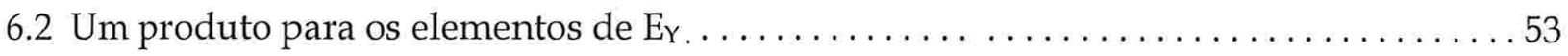

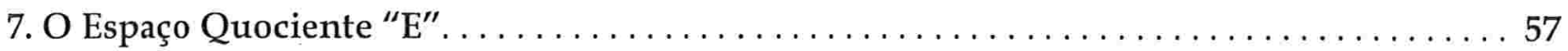

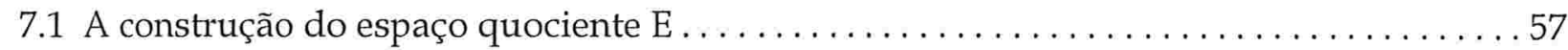

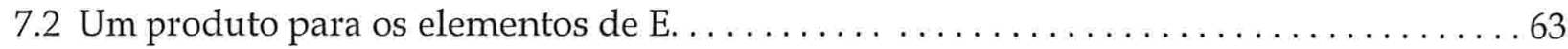

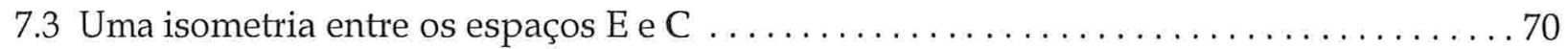

8. Operadores em Subespaços de Espaços de Banach Hereditariamente Indecomponíveis . . 81

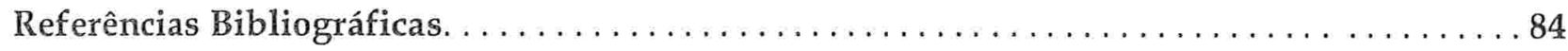




\section{INTRODUÇÃO}

Foi provado no artigo "The Unconditional Basic Sequence Problem" de W. T. Gowers e B. Maurey [6], que se $X$ é um Espaço de Banach Complexo Hereditariamente Indecomponível (H.I.), isto é, nenhum subespaço fechado de $X$ pode ser decomposto como soma direta topológica de dois subespaços fechados de dimensão infinita, então todo operador em $X$ é da forma $\lambda I+S$, onde $\lambda$ é um número complexo, I é identidade em $X$ e $S$ um operador estritamente singular.

Em 1996, V. Ferenczi, no artigo "Operators on subspaces of hereditarily indecomposable Banach spaces", publicado na Bull. London Math. Soc. [3], generalizou esse resultado, provando que se $X$ é um Espaço de Banach Complexo H.I., então para todo subespaço fechado $Y$ de $X$, com dimensão infinita, todo operador de $Y$ em $X$ é da forma $\lambda \mathrm{I}_{Y}+\mathrm{S}$ onde $\lambda$ é complexo, $\mathrm{I}_{Y}$ é a inclusão de $\mathrm{Y}$ em $X$ e $S$ um operador estritamente singular.

O principal objetivo dessa dissertação é estudar em detalhes a demonstração dada por Ferenczi e como conseqüência das técnicas desenvolvidas por esse matemático, também apresentamos um resultado importante no caso em que $X$ é um espaço de Banach real [4].

Vale ressaltar, que ainda não sabemos se o resultado anterior é verdadeiro quando $X$ é um espaço de Banach real, embora a maior parte da prova é válida para o caso real ou complexo. Mais precisamente, nós mostraremos que se $X$ é um espaço de Banach complexo H.I., então para todo $Y$ subespaço fechado de $X$, com dimensão infinita, o espaço quociente $\mathrm{L}(\mathrm{Y}, \mathrm{X}) / \mathrm{S}(\mathrm{Y}, \mathrm{X})$ com uma norma adequada é isométricamente isomorfo a uma Álgebra de Banach E. O teorema principal segue dessa propriedade e do Teorema de Gelfand - Mazur para o caso complexo (Teorema 2.2.3).

A maior parte dessa dissertação é destinada à construção do espaço $E$ e à demonstração de resultados importantes sobre esse espaço.

A seguir descreveremos os assuntos abordados em cada capítulo.

Inicialmente, no Capítulo 1, apresentaremos algumas definições e resultados básicos sobre Espaço Quociente, Bases de Schauder, Seqüências Básicas em Espaços de Banach, Espaços de Banach Hereditariamente Indecomponíveis e outros.

O Capítulo 2 traz a demonstração do Teorema de Gelfand-Mazur para os casos real e complexo. 
A seguir, no Capítulo 3, daremos a definição de Operadores Estritamente Singulares, Operadores Compactos, Operadores de Fredholm e demonstraremos alguns resultados importantes sobre esses operadores que serão utilizados nesta dissertação.

No próximo capítulo, definiremos o que é um $\mathrm{I}_{Y}+\mathrm{S}$ - isomorfismo e a partir dessa noção demonstraremos um lema que será fundamental para este trabalho.

A apresentação de uma conveniente semi-norma no espaço $L(Y, X)$ e a demonstração de algumas propriedades dessa semi-norma, será feita no Capítulo 5.

O Capítulo 6 apresenta o espaço quociente $E_{Y}$ e traz a definição de uma isometria linear entre os espaços $E_{Y}$ e $E_{Z}$. Essa isometria irá desempenhar um papel importante na construção do espaço quociente E.

A definição de "família coerente", a apresentação do espaço quociente E e a demonstração de que esse espaço possui uma estrutura de Álgebra de Banach, será feita no Capítulo 7.

O último capítulo traz a demonstração do principal teorema dessa dissertação, bem como uma nova caracterização para os espaços de Banach complexo hereditariamente indecomponíveis, obtida por Ferenczi. 


\section{CAPÍTULO 1}

\section{PRELIMINARES}

\subsection{Notações}

Denotaremos por $\mathrm{C}$ o conjunto dos números complexos, $\mathrm{R}$ o conjunto dos números reais, $\mathbf{R}_{+}$o conjunto dos números reais não negativos, $\mathbf{R}_{+}^{*}$ o conjunto dos números reais estritamente positivos, $\mathrm{N}$ o conjunto dos números naturais e IK os corpos $\mathrm{R}$ ou $\mathrm{C}$.

Sejam $X$ e $Y$ espaços normados. $L(Y, X)$ denotará o espaço de Banach dos operadores lineares e contínuos de $Y$ em $X$ com a norma ||$T||=\sup \{|| T(y)||:|y| \mid \leq 1\}$. Lembremos que um operador linear $\mathrm{T}$ é contínuo, se e somente se, existe $\mathrm{M}>0$ tal que || $\mathrm{T}(\mathrm{y})|| \leq \mathrm{M} .|| \mathrm{y}||, \forall \mathrm{y} \in \mathrm{Y}$.

Denotaremos por $\operatorname{ImT}$ a imagem do operador $\mathrm{T}$, isto é, $\operatorname{ImT}=\{\mathrm{T}(\mathrm{y}): \mathrm{y} \in \mathrm{Y}\}$ e por $\operatorname{Ker}(T)$, o kernel desse operador, ou seja, $\operatorname{Ker}(T)=\{y \in Y: T(y)=0\}$.

Um operador linear T $: Y \rightarrow X$ é um isomorfismo, se e somente se, existirem $M$ e $N$ números reais positivos, tais que $\mathrm{N}|| \mathrm{y}|| \leq|| \mathrm{T}(\mathrm{y})|| \leq \mathrm{M}|| \mathrm{y}||, \forall \mathrm{y} \in \mathrm{Y}$. Nesse caso dizemos que $Y$ e $X$ são isomorfos e denotamos $X=Y$. Sendo $W$ um subespaço de $Y$, denotaremos por $T_{\mid W}$ a restrição do operador $\mathrm{T}$ ao subespaço $\mathrm{W}$.

Finalmente, sendo $X$ um espaço vetorial normado, denotaremos a dimensão desse espaço por $\operatorname{dim} X$.

O símbolo 䊅 denota o fim de uma demonstração. 


\subsection{Definições e Resultados}

No que se segue estaremos apresentando algumas definições e resultados básicos envolvendo Espaços de Banach.

Definição 1.2.1 Seja X um espaço vetorial sobre um corpo $\mathrm{IK}$, a aplicação $p: X \rightarrow \mathbf{R}_{+}$é uma semi-norma sobre $X$ se :

- $p(\lambda x)=|\lambda| \cdot p(x) \quad \forall x \in X, \forall \lambda \in \mathrm{IK}$.

- $p(x+y) \leq p(x)+p(y) \quad \forall x, y \in X$.

Se $p(x)=0$ implicar $x=0$, então pé uma norma em $\mathrm{X}$.

Definição 1.2.2 Um espaço X é um espaço de Banach se :

- Xé um espaço vetorial.

- Xé normado.

- Xé completo em relação a essa norma.

Proposição 1.2.3 Se X é um espaço vetorial normado isomorfo a um espaço de Banach $Y$, então Xé um espaço de Banach.

Prova. Verificação imediata.

Definição 1.2.4 Uma seqüência $\left(x_{n}\right)_{n}$ é uma base de Schauder de um espaço de Banach X se, e somente se, para cada $x \in X$, existe uma única seqüiência $\left(a_{n}\right)_{n}$ de escalares tais que $x=\sum_{n=1}^{\infty} a_{n} x_{n}$.

Definição 1.2.5 Uma seqüência $\left(x_{n}\right)_{n}$ que é base de Schauder do subespaço fechado gerado por ela, isto é, $\left[\left(x_{n}\right)_{n}\right]$, é chamada seqüência básica. 
Proposição 1.2.6 Se $\left(x_{n}\right)_{n}$ é base de Schauder de $X$, então os operadores $P_{n}: X \rightarrow X$ tais que $P_{n}\left(\sum_{i=1}^{\infty} a_{i} x_{i}\right)=\sum_{i=1}^{n} a_{i} x_{i}$ são lineares e contínuos, e mais $\sup _{n \in N}|| P_{n}||<\infty$.

Prova. Sugerimos [10], pág. 1.

Definição 1.2.7 $k=\sup _{n \in N}|| P_{n}||$ é chamada constante básica de $\left(x_{n}\right)_{n}$.

Observação 1.2.8 Nem todo espaço de Banach possui base de Schauder. No entanto neste trabalho iremos trabalhar com seqüências básicas em espaços de Banach.

Proposição 1.2.9 $\left(x_{n}\right)_{n}$ é base de Schauder de um espaço de Banach X, se e somente se,

- $x_{n} \neq 0 \quad \forall n \in N$;

- $\exists M \in R_{+}^{*}$ tal que ||$\sum_{i=1}^{n} a_{i} x_{i}|| \leq M|| \sum_{i=1}^{n+p} a_{i} x_{i}||, \forall n, p \in N$;

- $\left[\left(x_{n}\right)_{n}\right]=X$.

Prova. Sugerimos [10], pág. 2.

Teorema 1.2.10 Fixado $k>1$, todo espaço de Banach de dimensão infinita possui uma seqüência básica com constante $k$.

Prova. Sugerimos [10], pág. 4.

Definição 1.2.11 Duas bases de Schauder $\left(x_{n}\right)_{n}$ e $\left(y_{n}\right)_{n}$ de espaços de Banach X e Y, respectivamente, são ditas equivalentes quando $\sum_{n=1}^{\infty} a_{n} x_{n}$ converge, se e somente se, $\sum_{n=1}^{\infty} a_{n} y_{n}$ converge.

Proposição 1.2.12 Sejam $\left(x_{n}\right)_{n}$ e $\left(y_{n}\right)_{n}$ bases de Schauder dos espaços de Banach X e Y, respectivamente, então são equivalentes :

a) $\left(x_{n}\right)_{n}$ é equivalente a $\left(y_{n}\right)_{n}$. 
b) Existe um isomorfismo $T: X \rightarrow Y$ tal que $T\left(x_{n}\right)=y_{n}, \forall n \in N$, onde para cada $x \in X$, $x=\sum_{i=1}^{\infty} a_{i} x_{i}$, temos $T(x)=\sum_{i=1}^{\infty} a_{i} y_{i}$.

Prova. Sugerimos [10], pág. 5.

Proposição 1.2.13 Seja $\left(x_{n}\right)_{n}$ uma seqüência básica normalizada de um espaço de Banach $X$, com constante básica $k$, e seja $\left(y_{n}\right)_{n}$ uma seqüência de vetores em $X$, tal que:

$$
\sum_{n=1}^{\infty}\left\|x_{n}-y_{n}\right\|<\frac{1}{2 k}
$$

Então $\left(y_{n}\right)_{n}$ é uma seqüência básica de $X$, equivalente $a\left(x_{n}\right)_{n}$.

Prova. Sugerimos [10], pág. 5.

Definição 1.2.14 Sejam $X$ um espaço de Banach, $\left(x_{n}\right)_{n}$ uma seqüência básica em $X,\left(p_{n}\right)_{n}$ uma seqüência estritamente crescente de inteiros positivos e $\left(a_{n}\right)_{n}$ uma seqüência de escalares. Uma seqüência $\left(u_{j}\right)_{j}$ dada por $u_{j}=\sum_{n=p_{j}+1}^{p_{j+1}} a_{n} x_{n}$, com $u_{j} \neq 0, \forall j \in N$, é chamada de base de bloco de $\left(x_{n}\right)_{n}$.

Proposição 1.2.15 Uma base de blocos $\left(u_{j}\right)_{j}$ de uma seqüência básica $\left(x_{n}\right)_{n}$ de um espaço de Banach $X$, é uma sequiência básica, cuja constante básica é menor ou igual a constante básica de $\left(x_{n}\right)_{n}$.

Prova. Sugerimos [10], pág. 6.

Proposição 1.2.16 Seja Y um subespaço de dimensão infinita de um espaço de Banach $X, e\left(x_{n}\right)_{n}$ base de Schauder de X. Então para todo inteiro positivo $p$, existe $y \in Y$, com ||$y||=1$ e da forma $y=\sum_{n=p+1}^{\infty} a_{n} x_{n}$

Prova. Sugerimos [5], pág. 52. 
Proposição 1.2.17 Seja $\left(x_{n}\right)_{n}$ base de Schauder, com constante $k$, de um espaço de Banach X, com $M_{1} \leq|| x_{n}|| \leq M_{2}, \forall n \in N$, onde $M_{1}$ e $M_{2}$ são constantes positivas. Então, dado $x=\sum_{n=1}^{\infty} a_{n} x_{n}$ temos $:\left|a_{n}\right| \leq \frac{2 k}{M_{1}} .\|x\|, \forall n \in N$.

Prova. Sugerimos [5], pág. 48.

Proposição 1.2.18 Se X é um espaço de Banach e $\left(x_{n}\right)_{n}$ base de Schauder de X, então $\left(\frac{x_{n}}{\left\|x_{n}\right\|}\right)_{n}$ é uma base normalizada de X com a mesma constante básica.

Prova. Segue diretamente da Proposição 1.2.9.

Proposição 1.2.19 Seja X um espaço vetorial normado. X é um espaço de Banach, se e somente se, toda série normalmente convergente é convergente em $\mathrm{X}$.

Prova. Sugerimos [11], pág. 67.

Definição 1.2.20 Um espaço métrico $M$ chama-se localmente compacto, quando todo $x \in M$ possui uma vizinhança compacta.

Proposição 1.2.21 Um espaço métrico $M$ é localmente compacto se, e somente se, para cada $x \in M$, existe $r>0$ tal que a bola fechada de centro em x e raio ré compacta.

Prova. Sugerimos [9], pág. 235.

Teorema 1.2.22 ( Teorema de Riesz) Um espaço normado é localmente compacto, se e somente se, for de dimensão finita.

Prova. Sugerimos [7], pág. 114. 
Teorema 1.2.23 (Teorema da Aplicação Aberta) Seja T uma aplicação linear contínua de um espaço de Banach $Y$ sobre um espaço de Banach X. Para todo conjunto aberto $W \subset Y, T(W)$ é aberto em X.

Prova. Sugerimos [7], pág. 245.

Corolário 1.2.24 Sejam Ye X espaços de Banach e $T \in L(Y, X)$ bijetora. Então Té bicontinua, isto é, $T^{-1}$ é contínua.

Prova. Sugerimos [7], pág. 245.

\subsection{Espaço Quociente}

Nesta seção apresentaremos a definição de espaço quociente e alguns resultados que serão utilizados em capítulos posteriores.

Definição 1.3.1 Seja X um espaço vetorial sobre um corpo IK e Y um subespaço de X. Vamos construir um novo espaço vetorial, chamado Espaço Quociente, o qual denotaremos por X / Y. Primeiramente vamos definir uma relação de equivalência nos elementos de X:

Dados $u, v \in X, u \sim v \Leftrightarrow u-v \in Y$.

É fácil verificar que é uma relação de equivalência, e portanto provoca uma partição em X.

Dado $\mathrm{x} \in \mathrm{X}$ denotaremos por $\bar{x}$ sua classe de equivalência,isto é $\bar{x}=\{\mathrm{y} \in \mathrm{X}: \mathrm{y} \sim \mathrm{x}\}$.

Denotando por $X / Y=\{\bar{x} / \mathrm{x} \in \mathrm{X}\}$ é fácil ver que $X / Y$ possui estrutura de espaço vetorial, basta definirmos em $X / Y$ as seguintes operações :

- $\bar{x}+\bar{y}=\overline{x+y} \quad, \forall \bar{x}, \bar{y} \in \mathrm{X} / \mathrm{Y}$.

- $\lambda \bar{x}=\overline{\lambda x} \quad, \forall \bar{x}, \in \mathrm{X} / \mathrm{Y}, \forall \lambda \in \mathrm{IK}$. 
É fácil provar que essas operações estão bem definidas, isto é, independem da escolha dos representantes.

Proposição 1.3.2 Dado um espaço vetorial normado $X$ e um subespaço vetorial $Y$ de $X$, a aplicação ||$.||$ dada por $\bar{x} \in X / Y \rightarrow|| \bar{x}||=\inf \{|| z||: z \in \bar{x}\}$,é uma semi-norma em $X / Y$.

Prova. Sugerimos [7], pág. 121.

Observação 1.3.3 A aplicação definida anteriormente é uma norma em $X / Y$, se e somente se, $Y$ é subespaço fechado de X.

Prova. Sugerimos [7], pág. 121.

Proposição 1.3.4 Seja X um espaço de Banach e Y um subespaço vetorial fechado de X. Então o espaço vetorial $X / Y$ com a norma definida anteriormente é completo.

Prova. Sugerimos [7], pág. 124.

Proposição 1.3.5 Seja $X$ um espaço vetorial com semi-norma || . ||, e $Y=\{x \in X:|| x||=0\}$, então Yé um subespaço vetorial de X, e sobre X/Y podemos definir a aplicação || $\bar{x}||=|| x||$ que é uma norma em X/Y.

Prova. Primeiramente, vamos provar que $Y$ é subespaço vetorial de $X$. De fato. É imediato que $0 \in \mathrm{Y}$. Agora, sendo $\mathrm{x}$ e $\mathrm{y} \in \mathrm{Y}, \lambda \in \mathrm{IK}$, e lembrando que ||. | é uma semi-norma em $X$ temos:

$$
\begin{aligned}
& || x+y|| \leq|| x||+|| y||=0 \Rightarrow|| x+y||=0 \Rightarrow x+y \in Y . \\
& || \lambda x||=|\lambda| .|| x||=0 \Rightarrow|| \lambda x||=0 \Rightarrow \lambda x \in Y .
\end{aligned}
$$

Vamos agora, mostrar que a aplicação ||$.||: X / X \rightarrow \mathbb{R}+$ está bem definida.

$$
\bar{x} \rightarrow|| \bar{x}||=|| x||
$$


Seja $\mathrm{z} \in \bar{x}$, então temos que $\mathrm{z}-\mathrm{x}=\mathrm{y} \in \mathrm{Y}$, ou seja, $\mathrm{z}=\mathrm{x}+\mathrm{y}$. Assim:

|| $\bar{x}||=|| z||=|| x+y|| \leq|| x||+|| y||=|| x||$, uma vez que ||$y||=0$.

Logo ||$z|| \leq|| x||$

Por outro lado, podemos escrever $\mathrm{x}=\mathrm{z}-\mathrm{y}$ e assim segue:

||$x||=|| z-y|| \leq|| z||+|| y||=|| z||$, ou seja, ||$x|| \leq|| z||$.

De (1) e (2) segue que ||$x||=|| z||$, ou seja, || $\bar{x}||=|| x||$ independe da escolha do representante, logo está bem definida.

Finalmente, vamos mostrar que a aplicação definida anteriormente é uma norma em X/Y.

Sendo $\bar{x}$ e $\bar{y} \in X / Y, \lambda \in$ IK e lembrando que ||.|| é uma semi-norma em $\mathrm{X}$, temos:

|| $\bar{x}+\bar{y}||=|| \overline{x+y}||=|| \mathrm{x}+\mathrm{y}|| \leq|| \mathrm{x}||+|| \mathrm{y}||=|| \bar{x}||+|| \bar{y}||$.

||$\lambda \bar{x}||=|| \overline{\lambda x}||=|| \lambda x||=|\lambda| .|| x||=|\lambda| .|| \bar{x}||$.

|| $\bar{x}||=0 \Rightarrow|| x||=0 \Rightarrow \mathrm{x} \in \mathrm{Y} \Rightarrow 0-\mathrm{x} \in \mathrm{Y} \Rightarrow 0 \in \bar{x} \Rightarrow \bar{x}=\overline{0}$

De (3), (4) e (5) decorre que | |. | | é uma norma em X/Y.

\subsection{Espaços de Banach Hereditariamente Indecomponíveis}

O objetivo dessa seção é definir espaços de Banach Hereditariamente Indecomponíveis e demonstrar uma proposição que nos fornece uma caracterização geométrica de tais espaços.

Definição 1.4.1 Sendo X e Y espaços de Banach definimos:

$X \oplus Y=\{(x, y): x \in X e y \in Y\}$ 
Observação 1.4.2 A aplicação || . ||$_{\infty}: X \oplus Y \rightarrow R_{+}$definida abaixo é uma norma em $X \oplus Y$.

$$
||(x, y)||_{\infty}=\max \{|| x||,|| y||\}
$$

Proposição 1.4.3 Denotando por $X \underset{\infty}{\oplus} Y$ o espaço $X \oplus Y$ equipado com a norma definida na observação anterior, temos que $X \oplus Y$ é um espaço de Banach.

Prova. Verificação imediata.

Definição 1.4.4 Sejam X um espaço de Banach e Y um subespaço vetorial de X. Dizemos que Yé complementado em $X$, se existe um subespaço vetorial $W$ de $X$ tal que :

a) $Y \cap W=\{0\}$.

b) $\forall x \in X, \exists ! y \in Y$ e $\exists ! w \in W: x=y+w$.

c) A aplicação linear $P_{1}: X \rightarrow Y$ definida por $P_{1}(x)=$ yé contínua.

d) A aplicação linear $P_{2}: X \rightarrow W$ definida por $P_{2}(x)=w$ é contínua.

\section{Observação 1.4 .5}

- Ye $W$ são chamados suplementares topológicos, e escrevemos $X=Y \oplus W$.

- $\quad P_{1}$ é chamada projeção de X sobre Ye vale:
a) $P_{1}^{2}=P_{1}$
b) ||$P_{1}|| \geq 1$
c) $\left(I-P_{1}\right)^{2}=I-P_{1}$.

Proposição 1.4.6 Se o subespaço vetorial Yé complementado em um espaço de Banach X, então Y é fechado.

Prova. Verificação Imediata.

Definição 1.4.7 Seja X um espaço de Banach. X é hereditariamente indecomponível (H.I.), se nenhum subespaço fechado de $\mathrm{X}$ pode ser decomposto como soma direta topológica de dois subespaços fechados de dimensão infinita.

Observação 1.4 .8 (Teorema de Gowers-Maurey): Em 1993, Gotvers e Maurey construíram o primeiro espaço de Banach H.I, veja [6]. 
Definição 1.4.9 Sejam Ye Z subespaços fechados de dimensão infinita,

$$
\{(Y, Z)=\inf \{|| z-y||:|| z||=|| y||=1, y \in Y e z \in Z\},
$$

é definido como sendo o ângulo entre os subespaços Ye Z.

Lema 1.4.10 Sejam Ye Z subespaços fechados de dimensão infinita de um espaço de Banach X, tal que $Y \cap Z=\{0\}$. Então $Y+Z$ é fechado, se e somente se, $\chi(Y, Z)>0$.

Prova. Vamos definir a seguinte aplicação:

$$
\mathrm{T}: \mathrm{Y} \underset{\infty}{\oplus} \mathrm{Z} \rightarrow \mathrm{Y}+\mathrm{Z} \text { dada por } \mathrm{T}(\mathrm{y}, \mathrm{z})=\mathrm{y}-\mathrm{z}
$$

(i) É fácil verificar que T está bem definida.

(ii) T é linear. De fato,

$\mathrm{T}((\mathrm{a}, \mathrm{b})+\lambda(\mathrm{c}, \mathrm{d}))=\mathrm{T}(\mathrm{a}+\lambda \mathrm{c}, \mathrm{b}+\lambda \mathrm{d})=\mathrm{a}+\lambda \mathrm{c}-(\mathrm{b}+\lambda \mathrm{d})=(\mathrm{a}-\mathrm{b})-\lambda(\mathrm{c}-\mathrm{d})=\mathrm{T}(\mathrm{a}, \mathrm{b})+\lambda \mathrm{T}(\mathrm{c}, \mathrm{d})$

$\forall(\mathrm{a}, \mathrm{b}),(\mathrm{c}, \mathrm{d}) \in \mathrm{Y} \underset{\infty}{\oplus} \mathrm{Z}$ e $\forall \lambda \in \mathrm{IK}$.

(iii) $\mathrm{T}$ é injetora, pois dado $(\mathrm{y}, \mathrm{z}) \in \operatorname{ker}(\mathrm{T})$ temos:

$T(y, z)=0 \Rightarrow y-z=0 \Rightarrow y=z$. Mas, por hipótese temos que $Y \cap Z=\{0\}, \log 0 y=z=0$

Assim temos que $\operatorname{ker}(T)=\{0\}$, o que implica $T$ ser injetora.

(iv) T é sobrejetora. De fato. Seja $\alpha \in Y+Z$, daí temos: $\alpha \in Y+Z \Rightarrow \alpha=y+z \Rightarrow \alpha=y-(-z)$.

Assim, considerando o elemento $(y,-z) \in Y \underset{\infty}{\oplus} Z$ temos $T(y,-z)=y-(-z)=\alpha$.

(v) T é contínua. De fato, segue da Observação 1.4.2 e de (ii) que:

|| $\mathrm{T}(\mathrm{y}, \mathrm{z})||=|| \mathrm{y}-\mathrm{z}|| \leq|| \mathrm{y}||+|| \mathrm{z}|| \leq||(\mathrm{y}, \mathrm{z})||_{\infty}+||(\mathrm{y}, \mathrm{z})||_{\infty}=2||(\mathrm{y}, \mathrm{z})||_{\infty}$.

Logo, ||$T(y, z)|| \leq 2||(y, z)||_{\infty}, \forall(y, z) \in Y \oplus Z$. 
(vi) $\mathrm{T}^{-1}$ é contínua. De fato. Sendo $\mathrm{Y}+\mathrm{Z}$ subespaço fechado do espaço de Banach $X$, segue que $Y+Z$ é um espaço de Banach. Assim por um Corolário do Teorema da Aplicação Aberta (Corolário 1.2.24), temos que T-1 é contínua.

Assim, $\exists \mathrm{k}>0$ tal que $\mathrm{k}||(\mathrm{y}, \mathrm{z})||_{\infty} \leq|| \mathrm{T}(\mathrm{y}, \mathrm{z})|| \forall(\mathrm{y}, \mathrm{z}) \in \mathrm{Y} \underset{\infty}{\oplus} \mathrm{Z}$.

Logo, $k \cdot \max \{|| y||,|| z||\} \leq|| y-z||$, em particular, tomando ||$y||=|| z||=1$ temos:

||$y-z|| \geq k>0 \Rightarrow|| y-z||>0 \forall y \in Y, \forall z \in Z$ tal que ||$y||=|| z||=1$.

Assim, $\inf \{|| y-z||: y \in Y, z \in Z$ e ||$y||=|| z||=1\}>0$.

Reciprocamente, considerando a mesma aplicação T definida anteriormente temos:
(i) T está bem definida
(ii) T é linear
(iii) T é injetora
(iv) T é sobrejetora
(v) T é contínua.

(vi) Vamos agora, provar a seguinte afirmação: $\gamma(Y, Z)>0$ implica $T^{-1}$ contínua.

Suponhamos que T não tem inversa contínua, então:

$$
\forall \mathrm{n}>0, \exists(\mathrm{y}, \mathrm{z}) \in \mathrm{Y} \underset{\infty}{\oplus} \mathrm{Z} \text { tal que } \mathrm{n}||(\mathrm{y}, \mathrm{z})||_{\infty}>|| \mathrm{T}(\mathrm{y}, \mathrm{z})||
$$

Assim, para cada $n>0$, podemos escolher $(y, z) \in Y \underset{\infty}{\oplus} Z$ com norma unitária, satisfazendo (*). Logo fazendo $n \rightarrow 0$, temos que existe uma seqüência $\left(y_{i}, z_{i}\right) \in Y \underset{\infty}{\oplus} Z$, com norma unitária satisfazendo (*). Assim segue que ||$y_{i}-z_{i}|| \rightarrow 0$ quando $i \rightarrow \infty$.

Agora, como ||$\left(\mathrm{y}_{\mathrm{i}}, \mathrm{z}_{\mathrm{i}}\right)||_{\infty}=1, \forall \mathrm{i} \in \mathrm{N}$, ou podemos escolher $\mathrm{i}_{\mathrm{j}} \in \mathrm{N}$ tal que ||$y_{i j}||=1, \forall \mathrm{j}$, ou existe $\mathrm{i}_{\mathrm{j}} \in \mathrm{N}$ tal que || $\mathrm{z}_{\mathrm{i}_{\mathrm{j}}}||=1, \forall \mathrm{j}$. Assim, supondo ||$y_{i_{j}}||=1, \forall \mathrm{j}$, temos:

$1=|| y_{i j}||=|| y_{i_{j}}-z_{i j}+z_{i j}|| \leq|| y_{i_{j}}-z_{i j}||+|| z_{i j}|| \Rightarrow 1-|| y_{i_{j}}-z_{i_{j}}|| \leq$ ||$z_{i j}|| \leq 1$

Agora, fazendo $\mathrm{j} \rightarrow \infty$, temos ||$y_{i j}-z_{i j}|| \rightarrow 0$ e portanto ||$z_{i j}|| \rightarrow 1$.

Sendo assim, podemos supor ||$z_{i j}|| \neq 0, \forall j \in N$. Daí segue: 


$$
\left\|y_{i j}-\frac{z_{i j}}{\left\|z_{i j}\right\|}\right\|=\left\|y_{i_{j}}-z_{i j}+z_{i j}-\frac{z_{i j}}{\left\|z_{i j}\right\|}\right\| \leq|| y_{i j}-z_{i j}||+\left\|z_{i j}-\frac{z_{i j}}{\left\|z_{i j}\right\|}\right\| .
$$

Agora, fazendo $\mathrm{j} \rightarrow \infty$, por (**) segue que: $\left\|y_{i_{j}}-\frac{z_{i j}}{\left\|z_{i j}\right\|}\right\| \rightarrow 0$.

Assim $\gamma(Y, Z)=0$, o que é um absurdo, pois por hipótese temos $\gamma(Y, Z)>0$.

Logo T-1 é contínua.

Segue das afirmações anteriores que T é um isomorfismo e portanto: $Y \oplus Z=Y+Z$.

Agora, como $\mathrm{Y} \underset{\infty}{\oplus} \mathrm{Z}$ é um Espaço de Banach (ver Proposição 1.4.3), segue que $Y+Z$ também é Banach e sendo $Y+Z$ subespaço do espaço de Banach $X$, concluímos que $Y+Z$ é fechado.

Proposição 1.4.11 (Caracterização Geométrica dos Espaços de Banach H.I.) Seja X um espaço de Banach. X é H.I., se e somente se, para quaisquer $Y$ e $Z$ subespaços fechados de dimensão infinita de $X$, e para qualquer $\varepsilon>0$, existe $y \in Y$ e $z \in Z$ com ||$y||=|| z||=1$ tal que ||$y-z||<\varepsilon$.

Prova. Suponhamos que existam $Y$ e $Z$, subespaços fechados de dimensão infinita de $X$ e $\varepsilon>0$, tal que $\forall \mathrm{y} \in \mathrm{Y}, \forall \mathrm{z} \in Z$, unitários, temos || $\mathrm{y}-\mathrm{z}|| \geq \varepsilon>0$.

Daí segue: (i) $Y \cap Z=\{0\}$, pois dado $x \neq 0, x \in Y \cap Z$ temos:

$$
\mathrm{X} \in \mathrm{Y} \cap \mathrm{Z} \Rightarrow \mathrm{X} \in \mathrm{Y} \text { e } \mathrm{x} \in \mathrm{Z} \Rightarrow|| \frac{\mathrm{x}}{\|\mathrm{x}\|}-\frac{\mathrm{x}}{\|\mathrm{x}\|} \|=0
$$

Mas por (*) temos: ||$\frac{x}{\|x\|}-\frac{x}{\|x\|}|| \geq \varepsilon \Rightarrow \varepsilon \leq 0$, o que é um absurdo. Logo, $x=0$.

(ii) Como ||$y-z|| \geq \varepsilon>0, \forall y \in Y, \forall z \in Z$, unitários, segue que $\gamma(Y, Z) \geq \varepsilon>0$. Logo pelo lema anterior temos que $Y+Z$ é fechado e assim considerando $W=Y+Z$ temos que $W$ é subespaço fechado de $X$ e pela primeira parte da demonstração do Lema 1.4.10, segue que $W=Y \oplus Z$, o que é um absurdo pois $X$ é H.I.

Reciprocamente, suponhamos que $X$ não seja H.I. Logo existe $W$ subespaço fechado de $X$, tal que $W=Y \oplus Z$, onde $Y$ e $Z$ são subespaços fechados de $W$ com dimensão infinita. Assim, pelo Lema 1.4.10 temos que $\gamma(Y, Z)>0$, o que é um absurdo, pois tomando $\varepsilon=\gamma(Y, Z)$, por hipótese teríamos que existem $\mathrm{y} \in \mathrm{Y} \mathrm{e} \mathrm{z} \in \mathrm{Z}$, unitários tais que || $\mathrm{y}-\mathrm{z}||<\varepsilon$. 


\section{O TEOREMA DE GELFAND-MAZUR}

Este capítulo tem como objetivo principal demonstrar o Teorema de Gelfand-Mazur sobre Álgebras normadas com divisão para o caso real e complexo. Esse teorema irá desempenhar um papel fundamental nessa dissertação.

\section{1 Álgebras Normadas}

Definição 2.1.1 Uma álgebra normada sobre um corpo IKé um espaço vetorial normado A, sobre IK, no qual definimos uma operação de multiplicação interna, satisfazendo os seguintes axiomas:
a) $(x y) z=x(y z), \forall x, y, z \in A$.
b) $x(y+z)=x y+x z e(y+z) x=y x+z x, \forall x, y, z \in A$.
c) $\lambda(x y)=(\lambda x) y=x(\lambda y), \forall x, y, z \in A$ e $\forall \lambda \in \mathrm{IK}$.
d) ||$x y|| \leq|| x|| .|| y||, \forall x, y \in A$.

Observações 2.1.2

a) Se $\mathrm{IK}=\mathrm{R}$, A é chamada álgebra normada real, se $\mathrm{IK}=\mathrm{C}$, álgebra normada complexa.

b) Uma álgebra normada é comutativa se $x y=y x \quad \forall x, y \in A$.

c) Se A é um espaço vetorial completo em relação à norma ||.| |, então dizemos que Aé uma Álgebra de Banach.

Definição 2.1.3 Um elemento e de uma álgebra normada A é chamado unidade de A se, e somente se, $\mathbf{e} \neq 0, \mathrm{e} x=x \mathrm{e}=x, \forall x \in A e|| \mathrm{e}||=1$.

Observações 2.1.4

a) Nós dizemos que A é uma álgebra normada com unidade se existe em A o elemento unidade.

b) É possivel mostrar que o elemento unidade quando existe é único.

c) Sendo A uma álgebra normada com unidade, denotaremos o elemento unidade por 1. 
Definição 2.1.5 Sejam A uma álgebra normada com unidade e $x \in A$. Dizemos que $x$ é invertível se existe $y \in A$ tal que $x y=1$ e $y x=1$. O elemento y será chamado inverso de $x$ e o denotaremos por $x^{-1}$.

Definição 2.1.5 Uma álgebra normada com divisão é uma álgebra normada A com unidade tal que todo elemento não nulo é invertível.

Definição 2.1.6 Sejam A e B álgebras sobre um corpo IK. Um homomorfismo de A em B é uma aplicação $\phi \in L(A, B)$ tal que:

$$
\phi(x y)=\phi(x) \cdot \phi(y), \forall x, y \in A .
$$

Definição 2.1.7 Sejam A e B álgebras sobre um corpo IK. Um monomorfismo de A em B é um homomorfismo injetivo de A em B.

\subsection{O Caso Complexo do Teorema de Gelfand - Mazur}

Definição 2.2.1 Seja A uma álgebra normada complexa com unidade e $x \in A$. Dizemos que $\lambda \in \mathrm{C} e ́$ valor espectral de $x$ se $x$ - $\lambda 1$ não é invertível. Denotaremos por $\sigma(A, x)$ o conjunto de todos os valores espectrais de $x$ e chamaremos $\sigma(A, x)$ de espectro de $x$.

Proposição 2.2.2 Seja A uma Álgebra de Banach complexa com unidade. Então para todo $x \in A$, $\sigma(A, x)$ é um subconjunto não vazio e compacto de $\mathrm{C}$.

Prova: Sugerimos [2], pág. 23.

Teorema 2.2.3 (Caso Complexo do Teorema de Gelfand-Mazur) Seja A uma Álgebra de Banach complexa com divisão, então A é isométricamente isomorfo a $\mathrm{C}$.

Prova. Para cada $\mathrm{x} \in \mathrm{A}$, temos pela Proposição 2.2.2 que $\sigma(\mathrm{A}, \mathrm{x}) \neq \varnothing$. Portanto existe $\lambda \in \mathrm{C}$ tal que $x-\lambda 1$ não é invertível. Mas por hipótese, $\mathrm{A}$ é uma álgebra com divisão, assim temos que todo elemento não nulo de $\mathrm{A}$ é invertível, logo segue que $x-\lambda \mathbf{1}=0$, ou seja, $x=\lambda \mathbf{1}$. 
Logo para cada $x \in A$ corresponde um número complexo $\lambda$, tal que $x=\lambda \mathbf{1}$. Afirmamos que tal $\lambda$ é único. De fato, suponhamos que $x=\lambda \mathbf{1}$ e $x=\mu 1$ então temos $(\lambda-\mu) .1=0$, mas sendo A uma Álgebra, segue que $\lambda-\mu=0$, ou seja $\lambda=\mu$.

Dessa forma podemos definir a aplicação $\rho: A \rightarrow C$ dada por $\rho(x)=\lambda, \forall x \in A$.

Vamos agora mostrar que a aplicação $\rho$ é um isomorfismo que preserva a norma.

$\forall \mathrm{x}, \mathrm{y} \in \mathrm{A}$ temos $\rho(\mathrm{x}+\mathrm{y})=\lambda$ onde $\lambda \in \mathrm{C}$ é tal que $\mathrm{x}+\mathrm{y}=\lambda \mathbf{1}$.

Mas sendo $\rho(x)=\lambda_{1}$ e $\rho(y)=\lambda_{2}$ temos $x=\lambda_{1} \mathbf{1}$ e $y=\lambda_{2} \mathbf{1}$, assim segue que:

$$
x+y=\left(\lambda_{1}+\lambda_{2}\right) \cdot 1
$$

Agora pela unicidade de $\lambda$, por (1) e (2) segue que $\lambda=\lambda_{1}+\lambda_{2}$. Logo:

$$
\rho(x+y)=\lambda=\lambda_{1}+\lambda_{2}=\rho(x)+\rho(y), \forall x, y \in A .
$$

Seja agora $x \in A$ e $\alpha \in C, \alpha \neq 0$ (o caso $\alpha=0$ é imediato), daí temos:

$$
\begin{aligned}
& \rho(\alpha x)=\lambda \Rightarrow \alpha x=\lambda 1 \Rightarrow x=\alpha^{-1}(\lambda 1) \Rightarrow x=\left(\alpha^{-1} \lambda\right) 1 . \\
& \rho(x)=\lambda_{1} \Rightarrow x=\lambda_{1} 1 .
\end{aligned}
$$

Pela unicidade de $\lambda_{1}$, por (3) e (4) segue que $\alpha^{-1} \lambda=\lambda_{1} \Rightarrow \lambda=\alpha \lambda_{1}$. Logo:

$$
\rho(\alpha x)=\lambda=\alpha \lambda_{1}=\alpha \rho(x) \forall x \in A, \forall \alpha \in C
$$

Assim mostramos que:

$$
\rho(x+y)=\rho(x)+\rho(y) \quad \forall x, y \in A \text { e } \rho(\alpha x)=\alpha \rho(x) \quad \forall x \in A, \forall \alpha \in C
$$

Logo $\rho$ é uma aplicação linear. 
$\rho$ é injetora, pois dado $x \in A$, temos que $\rho(x)=0$ implica $x=0$.

$\rho$ é sobrejetora, pois dado $\lambda \in \mathrm{C}$, basta considerarmos o elemento $\lambda 1=x \in A$.

Falta agora, mostrarmos que $\rho$ é uma isometria, isto é: $|\rho(x)|=|| x||, \forall x \in A$.

Seja $x \in A$, então existe $\lambda \in C$ tal que $x=\lambda 1$. Assim, pela Observação 2.1.3 temos:

$$
|| x||=|| \lambda 1||=|\lambda| .|| 1||=|\lambda| .1=|\lambda|
$$

$\operatorname{Assim}|\rho(x)|=|\lambda|=|| x||, \forall x \in$ A.

Mostramos então que $\rho$ é uma isometria linear de A sobre $\mathrm{C}$ e, portanto um isomorfismo de A sobre C. Logo, A é isométricamente isomorfo a C.

\subsection{O Caso Real do Teorema de Gelfand - Mazur}

Os conceitos e resultados apresentados na seção anterior, somente são válidos para álgebras complexas. É então necessário encontrarmos algum procedimento para encaixar uma álgebra real numa álgebra complexa. Vamos assim definir o que é uma complexificação de uma álgebra real para em seguida demonstrarmos o caso real do Teorema de GelfandMazur.

Definição 2.3.1 Seja A uma álgebra real. A complexificação $A_{C}$ de A é o conjunto Ax A para o qual definimos, para todo $a, b, c, d \in A, \alpha, \beta \in \mathbf{R}$, as seguintes operações:

a) $(a, b)+(c, d)=(a+c, b+d)$.

b) $(\alpha+\beta i)(a, b)=(\alpha a-\beta b, \alpha b+\beta a)$.

c) $(a, b)(c, d)=(a c-b d, a d+b c)$ 
Observação 2.3.2 É fácil verificar que Ac possui estrutura de álgebra complexa e que a aplicação $a \rightarrow(a, 0)$ é um monomorfismo de A em Ac.

Proposição 2.3.3 Seja A uma álgebra normada real, então temos:

(i) A tem unidade se e somente se $A_{\mathrm{C}}$ tem unidade. Se 1 é a unidade de $A$, então $(\mathbf{1}, 0)$ é a unidade de $A_{\mathrm{C}}$.

(ii) $a \in A$ é invertível se e somente se $(a, 0) \in A_{C}$ é invertível, e $(a, 0)^{-1}=\left(a^{-1}, 0\right)$.

Prova. Sugerimos [2], pág. 69.

Observação 2.3.4 Sendo A uma álgebra normada sobre $\mathbf{R}$, e 1 a unidade em A, podemos considerar que $\mathbf{R}$ está contido propriamente em $A$, pois é fácil verificar que a aplicação $\phi: \mathbf{R} \rightarrow A$, definida por $\phi(\alpha)=1 \alpha$ é um monomorfismo de $\mathbf{R}$ em A. Logo podemos escrever, por abuso de linguagem, $\alpha \in A$ ao invés de $1 \alpha \in A$.

Definição 2.3.5 Seja A uma álgebra normada real com unidade e $x \in A$. O espectro de x é o conjunto $\operatorname{Sp}(A, x)$ de números complexos definido por: $\operatorname{Sp}(A, x)=\sigma\left(A_{C_{1}}(x, 0)\right)$.

Proposição 2.3.6 Seja A uma álgebra normada real com unidade e $x \in A$. Então:

(i) Existem $\xi$ e $\eta \in \mathbf{R}$, tal que $\xi+i \eta \in S p(A, x)$.

(ii) Sejam $\xi$ e $\eta \in \mathbf{R}$. Então $\xi+i \eta \in S p(A, x)$ se, e somente se, $(\xi-x)^{2}+\eta^{2} \in A$ não é invertível.

Prova. Sugerimos [2], pág. 70.

Definição 2.3.7 Sejam 1, $i, j, k$ a base usual de vetores do $\mathbf{R}^{4}$, isto é, $\mathbf{1}=(1,0,0,0), i=(0,1,0,0)$, $j=(0,0,1,0), k=(0,0,0,1)$. A álgebra dos quatérnios sobre $\mathbf{R}$, denotada por $\mathbf{H}$ é o espaço vetorial $\mathbf{R}^{4}$ com o produto interno definido sobre os vetores da base da seguinte forma:

$$
\begin{aligned}
& \mathbb{1}^{2}=1, \quad 1 i=i 1=i, \quad 1 j=j \mathbb{1}=j, \quad 1 k=k 1=k, \\
& i^{2}=j^{2}=k^{2}=-1, \quad i j=-j i=k, \quad j k=-k j=i, \quad k i=-i k=j .
\end{aligned}
$$


Observação 2.3.8 Estendendo a multiplicação anterior, por linearidade, para as combinações lineares dos elementos da base e considerando a norma definida por $|x|=\left(\sum_{m=1}^{4} x_{m}^{2}\right)^{\frac{1}{2}}$, onde $x=\left(x_{1}, x_{2}, x_{3}, x_{4}\right) \in \mathbb{H}$, é possível mostrar através de cálculos rotineiros que $\mathbf{H}$ é uma álgebra sobre $\mathbf{R}$, e que $|x y|=|x||y|$ $\forall x, y \in \mathbf{H}$, e portanto que $\mathbf{H}$ é uma álgebra normada sobre sobre $\mathbf{R}$.

Observação 2.3.9 O elemento 1 é a unidade de $\mathbf{H}$, pois dado $x=\left(x_{1}, x_{2}, x_{3}, x_{4}\right) \in \mathbf{H}$ temos:

$1 x=(1,0,0,0)\left(x_{1}, x_{2}, x_{3}, x_{4}\right)=(1+0 i+0 j+0 k)\left(x_{1}+x_{2} i+x_{3} j+x_{4} k\right)=x_{1}+x_{2} i+x_{3} j+x_{4} k=x$ e de forma análoga podemos mostrar que $x \mathbf{1}=x$.

Observação 2.3.10 Dado $x=\left(x_{1}, x_{2}, x_{3}, x_{4}\right) \in \mathrm{H}$, consideremos o elemento $x^{*}=\left(x_{1},-x_{2},-x_{3},-x_{4}\right)$, então: $x x^{*}=\left(x_{1}, x_{2}, x_{3}, x_{4}\right)\left(x_{1}-x_{2},-x_{3},-x_{4}\right)=\left(x_{1}+x_{2} i+x_{3} j+x_{4} k\right)\left(x_{1}-x_{2} i-x_{3} j-x_{4} k\right)=1|x|^{2}+0 i+0 j+0 k=$ $|x|^{2}$. Assim, sendo $x \neq 0$, podemos considerar o elemento $x^{-1}=\frac{1}{|x|^{2}} x^{*}$ tal que $x x^{-1}=x \cdot\left(\frac{1}{|x|^{2}} x^{*}\right)=$ $\frac{1}{|x|^{2}}\left(x x^{*}\right)=1$. De forma análoga podemos mostrar que $x^{-1} x=1$. Logo $\mathrm{H}$ é uma álgebra normada sobre $\mathbf{R}$ com divisão.

Vamos agora provar que toda álgebra normada real, com divisão é isomorfa a $\mathbf{R}, \mathbf{C}$ ou $\mathbf{H}$.

Definição 2.3.11 Seja A uma álgebra normada sobre $\mathbf{R}$, com divisão, definimos então os seguintes subconjuntos de $A, Y=\left\{x \in A:-x^{2} \in R_{+}\right\}$e $Y_{1}=\left\{x \in A:-x^{2}=1\right\}$.

Lema 2.3.12 Seja A uma álgebra normada sobre $\mathbf{R}$, com divisão. Então temos:

(i) $\mathbf{R} \cap Y=\{0\}$ e $\forall \mathrm{a} \in \mathrm{A}, \exists$ ! y $\in \mathrm{Y}$ e $\exists$ ! $\xi \in \mathbb{R}$, tal que $\mathrm{a}=\xi+\mathrm{y}$;

(ii) $\mathrm{Y}$ é subespaço vetorial de $\mathrm{A}$ e portando $\mathrm{A}=\mathrm{R} \oplus \mathrm{Y}$.

(iii) Se $Y_{1} \neq \varnothing$, então $Y=R Y_{1}$, isto $e ́, Y=\left\{x \in A: x=\alpha y\right.$ onde $\alpha \in \mathbb{R}$ e y $\left.\in Y_{1}\right\}$.

Prova. (i) Seja a $\in$ A. Pela Proposição 2.3 .6 (i) existem $\xi, \eta \in \mathbb{R}$ tais que $\xi+$ i $\eta \in \operatorname{Sp}(A, a)$ e pela Proposição 2.3 .6 (ii) temos ainda que $(\xi-a)^{2}+\eta^{2}$ não é invertível em A. Mas, sendo A uma álgebra com divisão, segue que $(\xi-a)^{2}+\eta^{2}=0$, e portanto $-(\xi-a)^{2}=\eta^{2} \in \mathbb{R}_{+}, \operatorname{logo}$ $a-\xi \in Y$. 
Assim provamos que $\mathrm{a}-\xi=\mathrm{y} \in \mathrm{Y}$, ou seja, dado $\mathrm{a} \in \mathrm{A}$, temos $\mathrm{a}=\xi+\mathrm{y}$ onde $\xi \in \mathbb{R}$ e y $\in \mathrm{X}$. Vamos agora mostrar que se $\mathbf{a}=\xi^{\prime}+\mathrm{y}^{\prime} \operatorname{com} \xi^{\prime} \in \mathbf{R}$ e $y^{\prime} \in Y$, então $\xi^{\prime}=\xi$ e $y^{\prime}=y$. De fato.

$$
a=\xi^{\prime}+y^{\prime} \Rightarrow y^{\prime}=a-\xi^{\prime} \text {, mas como } a=\xi+y \text {, segue que } y^{\prime}=\left(\xi-\xi^{\prime}\right)+y \text {, }
$$

e por tanto $\left(y^{\prime}\right)^{2}=\left(\xi-\xi^{\prime}\right)^{2}+2\left(\xi-\xi^{\prime}\right) y+y^{2}$.

Agora, como $\mathrm{y}^{\prime} \in \mathrm{Y}$, temos que $-\left(\mathrm{y}^{\prime}\right)^{2} \in \mathbf{R}_{+}$, ou seja, $-\left(\xi-\xi^{\prime}\right)^{2}-2\left(\xi-\xi^{\prime}\right) \mathrm{y}-\mathrm{y}^{2} \in \mathbf{R}_{+}$. Mas, como $\left(\xi-\xi^{\prime}\right)^{2} \in \mathbf{R}$ e $-y^{2} \in \mathbf{R}_{+}$, segue que $\left(\xi-\xi^{\prime}\right) y \in \mathbf{R}$, portanto $\xi-\xi^{\prime}=0$ ou $y \in \mathbf{R}$. Se y $\in \mathbf{R}$,

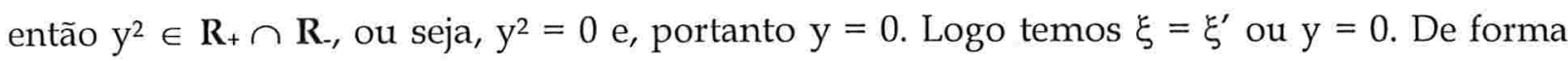
análoga, isolando y em (1) temos que $\xi^{\prime}=\xi$ ou $\mathrm{y}^{\prime}=0$, e assim provamos que a $\in$ A se escreve de forma única como soma de um elemento de $\mathbf{R}$ e um elemento de $Y$ e que $R \cap Y=\{0\}$.

(ii) Vamos agora mostrar que $\mathrm{Y}$ é subespaço vetorial de A.

- Seja $\alpha \in R$ e $y \in Y$, então $\alpha y \in Y$. De fato, $-(\alpha y)^{2}=-\alpha^{2} y^{2}=\alpha^{2}\left(-y^{2}\right)$ onde $\alpha^{2} \in R_{+}$e $-y^{2} \in \mathbf{R}_{+}$, uma vez que $y \in Y$.

- Dados $x, y \in Y$, vamos agora mostrar que $x+y \in Y$.

Sendo $x, y \in Y$, temos que $x, y \in A$, logo $x+y \in A$ e $x-y \in A$, e por (i) podemos escrever:

$$
x+y=\alpha+w \text { e } x-y=\beta+z \text { onde } \alpha, \beta \in R \text { e } w, z \in Y
$$

Assim, $2 x^{2}+2 y^{2}=(x+y)^{2}+(x-y)^{2}=\alpha^{2}+\beta^{2}+2 \alpha w+2 \beta z+w^{2}+z^{2}$.

Agora, como $x, y, w, z \in Y$ temos que $x^{2}, y^{2}, z^{2}$ e $w^{2} \in R$ e sendo $\alpha, \beta \in R$, segue de (3) que $\alpha w+\beta z=\lambda \in \mathbb{R}, \operatorname{assim} \lambda-\alpha w=0+\beta z$.

Mas por (i) segue que $\lambda=0$, ou seja, $\alpha w+\beta z=0$.

Portanto, segue de (2) e (4) que: 
$(\alpha+\beta) x+(\alpha-\beta) y=\alpha(x+y)+\beta(x-y)=\alpha(\alpha+w)+\beta(\beta+z)=\alpha^{2}+\alpha w+\beta^{2}+\beta z=\alpha^{2}+\beta^{2}$, ou seja, $0+(\alpha+\beta) x=\left(\alpha^{2}+\beta^{2}\right)+(\beta-\alpha) y$, e então utilizando mais uma o que foi provado em (i), segue que $\alpha^{2}+\beta^{2}=0$. Agora, como $\alpha$ e $\beta$ são números reais, temos $\alpha=\beta=0$ e portanto por (2) concluímos que $x+y=w \in Y$. Assim temos que $Y$ é subespaço vetorial de A e por (i) segue que $A=R \oplus Y$.

(iii) Seja a $\in \mathbf{R} Y_{1}$, então $a=\alpha y_{1}$ onde $\alpha \in \mathbf{R}$ e $y_{1} \in Y_{1}$. Assim, $a^{2}=\alpha^{2}\left(y_{1}\right)^{2}$ onde $-\left(y_{1}\right)^{2}=1$, $\operatorname{logo} a^{2}=-\alpha^{2}$, ou seja, $-a^{2}=\alpha^{2} \in R_{+}$, portanto $a \in Y$. Por outro lado, seja $y \in Y$, então $-y^{2}=\beta^{2} \in \mathbf{R}_{+}$. Supondo $\beta \neq 0$, temos $-y^{2}\left(\beta^{2}\right)^{-1}=1$, ou seja , y $\beta^{-1}=y_{1} \in Y_{1}$ e por tanto $y=\beta y_{1} \in R Y_{1}$. O caso onde $\beta=0$ é imediato, uma vez que se $\beta=0$ então $y=0$ e assim $y=0 y_{1}$ $\forall \mathrm{y}_{1} \in \mathrm{Y}_{1}$.

Lema 2.3.13 Sejam $u \in Y_{1} e \quad W(u)=\{x \in Y: u x \in Y\}$. Então $Y=\mathbf{R} u \oplus W(u)$, onde $\mathbf{R} u=\{\alpha u: \alpha \in \mathbf{R}\}$.

Prova. Seja $\mathrm{x} \in \mathrm{Y}$, pelo Lema 2.3.12 temos que $\mathrm{ux}=\lambda+\mathrm{y}$ ou $\mathrm{ux}-\lambda=\mathrm{y} \operatorname{com} \lambda \in \mathrm{R}$ e $\mathrm{y} \in \mathrm{Y}$.

Agora, lembrando que $\mathrm{u}^{2}=-1$ segue de $(1): \mathrm{u}(\mathrm{x}+\lambda \mathrm{u})=\mathrm{ux}+\lambda \mathrm{u}^{2}=\mathrm{ux}-\lambda=\mathrm{y} \in \mathrm{Y}$, e sendo $\mathrm{Y}$ subespaço linear, como $u=1 u \in Y$, pois $Y=R Y_{1}$, temos que $x+\lambda u \in Y$ e por tanto $x+\lambda u \in W(u)$, ou seja $x+\lambda u=w \in W(u)$, isto é, $x=w+(-\lambda u)$. Assim mostramos que $x \in \mathbf{R u}+W(u)$. Suponha agora que $x=w^{\prime}+\beta u$ para algum $\beta \in \mathbf{R}$ e $w^{\prime} \in W(u)$, então temos:

$$
x=w+(-\lambda u) \Rightarrow u x=u w+\lambda \quad \text { e } \quad x=w^{\prime}+(-\beta u) \Rightarrow u x=u w^{\prime}+\beta
$$

Mas, novamente pelo Lema 2.3.12 temos que ux se escreve de forma única como soma de um elemento de $\mathbf{R}$ e um elemento de $Y, \log \alpha=\beta$ e uw $=u^{\prime}$, ou seja, $\lambda=\beta$ e $w=w^{\prime}$. Por tanto mostramos que $Y=R u \oplus W(u)$.

Lema 2.3.14 Sejam $u, v \in Y_{1} e v \in W(u)$. Então $u v \in Y_{1}$ e vu $=-u v$.

Prova. Sendo $v \in W(u)$, nos temos que $u v \in Y$, assim (uv)(uv) $=-\lambda \operatorname{com} \lambda \in \mathbb{R}_{+}$, e lembrando que $u^{2}=-1$ e $v^{2}=-1$, temos: $v u=u^{2}(v u) v^{2}=u(u v u v) v=u(-\lambda) v=-\lambda u v$, 
Agora, como $u, v \in Y_{1}$ temos que $1 \mathrm{u}, 1 \mathrm{v}$ e $-1 v \in Y$ pois $Y=R Y_{1}$. Assim, sendo $Y u m$ subespaço vetorial (ver Lema 2.3.12) segue que $u+v, u-v \in Y$ e portanto $(u+v)^{2} e$ $(u-v)^{2} \in \mathbf{R}$.

E verificando que $2(u v+v u)=(u+v)^{2}-(u-v)^{2}$, segue de (1) e (2) que:

$$
(1-\lambda) \mathrm{uv} \in \mathbf{R} \text {. }
$$

Por outro lado, $u v \neq 0$. De fato, como $u^{2}=v^{2}=-1$ temos $u^{2} v^{2}=1$, ou seja, $u(u v) v=1$, daí, se uv $=0$ teríamos $0=1$, o que é um absurdo. Assim, como $Y \cap R=\{0\}$ (ver Lema 2.3.12) e $u v \neq 0$, temos que uv $\notin \mathbf{R}$, daí segue de (3) que $1-\lambda=0$, ou seja, $\lambda=1$. Logo temos por (1) que $u v=-$ vu e $(u v)^{2}=-1$, e portanto $u v \in Y_{1}$.

Teorema 2.3.15 (O caso real do Teorema de Gelfand - Mazur) Seja A uma álgebra normada real, com divisão. Então Aé isomorfo a $\mathbf{R}, \mathrm{C}$ ou $\mathbf{H}$.

Prova. Sendo $Y=\left\{x \in A:-x^{2} \in R_{+}\right\}$e $Y_{1}=\left\{x \in A:-x^{2}=1\right\}$, consideremos os seguintes casos:

(i) Suponha que $Y=\{0\}$, então lembrando que $A=R \oplus Y$ (ver Lema 2.3.12), segue imediatamente que $A=R$, ou seja, $A$ é isomorfo a $\mathbf{R}$.

(ii) Supondo que $\operatorname{dim} Y=1$, como $Y=R Y_{1}$ (ver Lema 2.3.12), podemos tomar $i \in Y_{1}$ tal que $Y=R i$, onde $i^{2}=-1$, e como $A=R \oplus Y($ Lema 2.3.12) temos que $A=R \oplus R i$, ou seja, $A$ é isomorfo a $\mathrm{C}$, uma vez que $\mathrm{C}=\mathrm{R} \oplus \mathrm{Ri}$.

(iii) Vamos agora supor que $\operatorname{dim} Y \geq 2$.

Dado $i \in Y_{1}$ e $W(i)=\{x \in Y: i x \in Y\}$, pelo Lema 2.3.13 temos que $Y=\mathbb{R i} \oplus W(i), \operatorname{logo}$ $W(i) \neq\{0\}$, pois caso contrário, teríamos $Y=R i$ e portanto $\operatorname{dim} Y=1$, o que contraria nossa hipótese inicial de que $\operatorname{dim} Y \geq 2$. Assim, podemos escolher $w \in W(i) \operatorname{com} w \neq 0$ e $w \neq i$. Mas como $w \in W(i) \Rightarrow w \in Y$ e $Y=\mathbb{R} Y_{1}$ (Lema 2.3.12) então $w=\operatorname{ry}_{1}$ onde $r \in \mathbb{R}, r \neq 0$ e $y_{1} \in Y_{1}$, assim podemos escrever $r^{-1} w=y_{1}$. Agora, como $i w \in Y$, pois $w \in W(i)$, e $Y$ é subespaço 
vetorial (Lema 2.3.12), podemos concluir que $\mathrm{i}\left(\mathrm{r}^{-1} \mathrm{w}\right)=\mathrm{r}^{-1}(\mathrm{i} w) \in \mathrm{Y}$, ou seja $\mathrm{y}_{1}=\mathrm{r}^{-1} \mathrm{w} \in \mathrm{W}(\mathrm{i})$. Logo mostramos que $W(i) \cap Y_{1} \neq \varnothing$.

Seja então $j \in W(i) \cap Y_{1}$, daí temos: $i, j \in Y_{1}$ e $j \in W(i)$, assim segue pelo Lema 2.3.14, que $i j=k \in Y_{1}$ e ji $=-k$. E também:

$$
j k=j(i j)=-j(j i)=-j^{2} i=i, k j=(i j) j=i j^{2}=-i .
$$

Similarmente temos $k i=-i k=j$. Nós provaremos agora que se $L=W(i) \cap W(j) \cap W(k)$, então $\mathrm{Y}=\mathrm{Ri} \oplus \mathbf{R j} \oplus \mathbf{R k} \oplus \mathrm{L}$.

De fato, pelo Lema 2.3.13, $Y=\mathbf{R i} \oplus W(\mathrm{i})=\mathbf{R} \mathbf{j} \oplus W(\mathrm{j})=\mathbf{R k} \oplus W(\mathrm{k})$.

Assim, dado $y \in Y$ temos:
a) $y=\alpha i+$ a onde $\alpha \in R$ e a, ia $\in Y$;
b) $a=\beta j+b$ onde $\beta \in R$ e b, $j b \in Y$;
c) $\mathrm{b}=\gamma \mathrm{k}+\mathrm{c}$ onde $\gamma \in \mathbf{R}$ e c, kc $\in \mathrm{Y}$.

Logo, segue por (a), (b) e (c) que $y=\alpha i+\beta j+\gamma k+c$, onde $c=b-\gamma k=a-\beta j-\gamma k$.

Assim, por (2) e (1) temos:

ic $=i a-\beta i j-\gamma i k=i a-\beta k+\gamma j$ com ia, $\beta k$ e $\gamma j \in Y$, ou seja, $c \in W(i)$.;

$j c=j b-\gamma j k=j b-\gamma i \operatorname{com} j b,-\gamma i \in Y$, ou seja, $c \in W(j)$;

$k c \in Y$, ou seja, $c \in W(k)$.

Logo, $\mathrm{c} \in \mathrm{L}$, e portanto mostramos que $\mathrm{Y}=\mathrm{Ri}+\mathrm{Rj}+\mathrm{Rk}+\mathrm{L}$.

Vamos agora motrar que a soma acima, é uma soma direta. Suponha que existam $\lambda, \mu, \eta$ e $c^{\prime} \in \mathbb{R}$, tais que $y=\lambda i+\mu j+\eta k+c^{\prime}$. Por (2) podemos escrever:

$$
(\alpha-\lambda) i+(\beta-\mu) j+(\gamma-\eta) k+\left(c-c^{\prime}\right)=0 .
$$


Assim, multiplicando a igualdade (3) por i, e utilizando (1), temos:

$(\lambda-\alpha)+(\beta-\mu) k-(\gamma-\eta) j+\left(c-c^{\prime}\right) i=0$, ou seja, $\quad(\lambda-\alpha)=-(\beta-\mu) k+(\gamma-\eta) j-\left(c-c^{\prime}\right) i \in Y$, uma vez que $(\beta-\mu) k,(\gamma-\eta)$ j e $\left(c-c^{\prime}\right) i \in Y$.

Logo, $\lambda-\alpha \in Y \cap R$, e então pelo Lema 2.3 .12 segue que $\lambda-\alpha=0$, isto é, $\lambda=\alpha$.

De forma análoga, podemos mostrar que $\beta=\mu, \gamma=-\eta$ e $c=c^{\prime}$.

Por tanto $\mathrm{Y}=\mathbf{R i} \oplus \mathbf{R} \mathbf{j} \oplus \mathbf{R} \mathrm{k} \oplus \mathrm{L}$.

Provaremos agora que $\mathrm{L}=\{0\}$.

De fato, pois caso contrário, podemos escolher $x \in L \cap Y_{1}$, e então pelo Lema 2.3.14, teremos:

$$
i x, j x, k x \in Y_{1}, i x=-x i, j x=-x j \text { e } k x=-x k \text {. }
$$

Assim, $x k=x(i j)=(x i) j=-(i x) j=-i(x j)=i(j x)=(i j) x=k x=-x k$, ou seja, $2 x k=0$, e portanto $\mathrm{xk}=0$, o que contradiz a hipótese de que $\mathrm{xk} \in \mathrm{Y}_{1}$.

Logo, $L=\{0\}$ e então segue diretamente por (4) que $\mathrm{Y}=\mathbf{R i} \oplus \mathbf{R j} \oplus \mathbf{R k}$. Agora, lembrando que $A=R \oplus Y($ Lema 2.3.12) temos $A=R \oplus \mathbf{R i} \oplus \mathbf{R j} \oplus \mathbf{R k}$, ou seja, $A=\mathbf{H}$, isto é, $A$ é isomorfo a álgebra dos quatérnios.

Assim, provamos em (i), (ii) e (iii) que sendo A, uma álgebra normada real, com divisão, então A será isomorfa a R, C ou $\mathbf{H}$. 


\section{OPERADORES ESTRITAMENTE SINGULARES OPERADORES COMPACTOS OPERADORES DE FREDHOLM}

Neste capítulo daremos algumas definições e resultados envolvendo operadores estritamente singulares, operadores compactos e operadores de Fredholm.

\subsection{Operadores Estritamente Singulares}

Definição 3.1.1 Sejam X e Y espaços normados com dimensão infinita. Seja $T: X \rightarrow Y$ linear e contínua. Dizemos que T é estritamente singular se a restrição de T a qualquer subespaço fechado $W$ de $X, W$ de dimensão infinita, não é um isomorfismo sobre a imagem.

Observações 3.1.2

- Denotaremos por $S(X, Y)$ o espaço das aplicações lineares e contínuas $S: X \rightarrow Y$, que são estritamente singulares.

- Depois da Proposição 3.1.4 será fácil demonstrar que $S(X, Y)$ é subespaço de $L(X, Y)$.

Vamos agora fazer uma caracterização dos operadores estritamente singulares.

Proposição 3.1.3 Sejam X e Y espaços normados com dimensão infinita. Um operador $T: X \rightarrow Y e ́$ estritamente singular se e somente se, $\forall \varepsilon>0$, para qualquer $W$ subespaço de dimensão infinita de $X$, existe $x \in W$ tal que ||$T(x)||<\varepsilon|| x||$.

Prova. Sabemos que se $\mathrm{T}: \mathrm{X} \rightarrow \mathrm{Y}$ é um isomorfismo então existem números reais $\mathrm{M}, \mathrm{N}>0$ tais que:

$$
\mathrm{M}|| \mathrm{x}|| \leq|| \mathrm{T}(\mathrm{x})|| \leq \mathrm{N}|| x||, \forall x \in X
$$


Seja $W$ um subespaço de $X$ com dimensão infinita. Então $T_{\mathrm{IW}_{\mathrm{W}}}$ é contínua , logo :

$$
\exists N>0:|| T(x)|| \leq N|| x||, \forall x \in W
$$

Agora como por hipótese $\mathrm{T}$ é estritamente singular, $\mathrm{T}_{\mathrm{lW}}$ não é um isomorfismo. Assim por (1) e (2) temos:

$$
\forall \varepsilon>0, \exists x \in W: \varepsilon|| x||>|| T(x)||
$$

Reciprocamente, suponhamos que T não seja estritamente singular, logo existe $W$ subespaço de dimensão infinita de $X$, tal que $T: W \rightarrow T(W)$ é isomorfismo. Assim por (1)

$$
\exists \mathrm{M}>0: \mathrm{M}|| \mathrm{x}|| \leq|| \mathrm{T}(\mathrm{x})||, \forall \mathrm{x} \in \mathrm{W},
$$

o que é um absurdo, pois por hipótese $\forall \mathrm{M}>0, \exists \mathrm{x} \in \mathrm{W}:|| \mathrm{T}(\mathrm{x})||<\mathrm{M}|| \mathrm{x}||$.

Proposição 3.1.4 Sejam X e Y espaços de Banach com dimensão infinita. Seja S: X $\rightarrow$ Y linear e contínua. Sé estritamente singular se, e somente se, para todo subespaço de dimensão infinita $W$ de $X$ e para todo $\varepsilon>0$, existe $W^{\prime}$ subespaço de dimensão infinita de $W$, tal que || $\mathrm{S}_{\mathrm{IW}}|| \leq \varepsilon$.

Prova. Sugerimos [10], pág. 76.

Corolário 3.1.5 Sejam $S_{1}$ e $S_{2} \in S(X, Y)$ e $\alpha \in \mathrm{IK}$. Então temos:

a) $S_{1}+S_{2} \in S(X, Y)$;

b) $\alpha S_{1} \in S(X, Y)$.

Prova. (a) Sejam $W$ subespaço de $X$ de dimensão infinita e $\varepsilon>0$. Por hipótese $S_{1}$ é estritamente singular, logo pela Proposição 3.1.4, existe $W^{\prime}$ subespaço de $W$, de dimensão infinita, tal que:

$$
|| S_{1 ! W},|| \leq \frac{\varepsilon}{2} \text {. }
$$


Agora, dado $W^{\prime}$ subespaço de $X$ e o mesmo $\varepsilon>0$, como $S_{2}$ também é estritamente singular, pela Proposição 3.1.4, existe $W^{\prime \prime}$ subespaço de $W^{\prime}$, de dimensão infinita, tal que:

$$
|| S_{2 \mid W^{*}}|| \leq \frac{\varepsilon}{2} \text {. }
$$

Assim, lembrando que $W^{\prime \prime} \subseteq W^{\prime}$, segue de (1) e (2) que:

$$
||\left(S_{1}+S_{2}\right)_{\mid W^{\prime \prime}}|| \leq|| S_{1_{\mid W^{\prime \prime}}}||+|| S_{2}\left|W^{\prime \prime}\right||\leq|\left|S_{1 \mid W^{\prime}}\right||+|\left|S_{2 \mid W^{\prime \prime}}\right| \mid \leq \frac{\varepsilon}{2}+\frac{\varepsilon}{2}=\varepsilon .
$$

Logo, dado $W$ subespaço de $X$, de dimensão infinita e $\varepsilon>0$, mostramos que existe $W^{\prime \prime}$ subespaço de $W$, de dimensão infinita, tal que ||$\left(S_{1}+S_{2}\right)_{\mid W^{\prime \prime}}|| \leq \varepsilon$. Assim, segue da Proposição 3.1.4 que $S_{1}+S_{2} \in S(X, Y)$.

Deixamos como exercício a prova da afirmação (b).

Proposição 3.1.6 Seja S um operador estritamente singular. Sejam T e U operadores lineares e contínuos, para os quais TS e SU estão definidas. Então TS e SU são operadores estritamente singulares.

Prova. Suponha que SU não seja estritamente singular. Então existe W subespaço fechado de $X, W$ de dimensão infinita, tal que $\mathrm{SU}: \mathrm{W} \rightarrow \mathrm{SU}(\mathrm{W})$ é isomorfismo. Logo:

$\exists \mathrm{M}, \mathrm{N}>0: \mathrm{N}|| \mathrm{x}|| \leq|| S U(x)|| \leq \mathrm{M}|| x||, \forall x \in W$

Sendo U linear e contínua $\exists K>0:|| U(x)|| \leq K|| x|| \forall x \in X$.

Em particular || $\mathrm{U}(\mathrm{x})|| \leq \mathrm{K}|| \mathrm{x}||, \forall \mathrm{x} \in \mathrm{W}$ implica $\frac{\|U(x)\|}{K} \leq|| \mathrm{x}||, \forall \mathrm{x} \in \mathrm{W}$.

De (1) e (2) segue que $\frac{N}{K}|| \mathrm{U}(\mathrm{x})|| \leq \mathrm{N}|| \mathrm{x}|| \leq|| \mathrm{SU}(\mathrm{x})|| \quad \forall \mathrm{x} \in \mathrm{W}$.

Por outro lado, ||$S U(x)||=|| S(U(x))|| \leq|| S|| .|| U(x)||, \forall x \in W$.

Assim pondo $K_{1}=\frac{N}{K}, K_{2}=|| S||$ e $U(x)=y$, por (3) e (4) temos : 
$\exists K_{1}, K_{2}>0: K_{1}|| y|| \leq|| S(y)|| \leq K_{2}|| y|| \quad \forall y \in Y_{1}$, onde $Y_{1}=U(W)$.

Logo $S: Y_{1} \rightarrow S\left(Y_{1}\right)$ é um isomorfismo.

Afirmação: $Y_{1}$ é subespaço fechado de $Y$ e tem dimensão infinita.

De fato. Suponha que $\mathrm{Y}_{1}=\mathrm{U}(\mathrm{W})$ seja de dimensão finita.Por (5) teríamos que $S\left(\mathrm{Y}_{1}\right)$ também teria dimensão finita, o que é um absurdo, pois $S\left(Y_{1}\right)=S(U(W))=S U(W)$ e $\mathrm{S}: \mathrm{W} \rightarrow \mathrm{SU}(\mathrm{W})$ é isomorfismo onde $\mathrm{W}$ é e fechado e tem dimensão infinita, logo $\mathrm{SU}(\mathrm{W})$ também é fechado e com dimensão infinita. Agora sendo $S\left(Y_{1}\right)=S(U(W))$ fechado, segue também de (5), que $Y_{1}$ é fechado.

Logo, exibimos $Y_{1}$, subespaço fechado de $Y$, com dimensão infinita, tal que $S: Y_{1} \rightarrow S\left(Y_{1}\right)$ é um isomorfismo, o que contraria nossa hipótese inicial de que $S$ é estritamente singular.

Portanto SU é um operador estritamente singular.

A demonstração de que TS é estritamente singular é feita de forma análoga.

Proposição 3.1.7 S(X,Y) é um subespaço fechado de $L(X, Y)$.

Prova. Como o Corolário 3.1.5 já nos garante que $S(X, Y)$ é um subespaço de $L(X, Y)$, basta mostrarmos que $S(X, Y)$ é fechado, isto é, que $S(X, Y)$ contém o limite de toda sequiência convergente em $S(X, Y)$.

Seja $T_{n} \in S(X, Y)$ e suponhamos que $T_{n} \rightarrow T$. Então:

Dado $\varepsilon>0, \exists \mathrm{n}_{0} \in \mathrm{N}: \mathrm{n} \geq \mathrm{n}_{0} \Rightarrow|| \mathrm{T}_{\mathrm{n}}-\mathrm{T}|| \leq \frac{\varepsilon}{2}$.

Pela Proposição 3.1.4, $\forall X^{\prime}$ subespaço de $X$, existe $X^{\prime \prime}$ subespaço de $X^{\prime}$ tal que:

$$
|| \mathrm{T}_{\mathrm{n}_{\mid \mathrm{X}}}|| \leq \frac{\varepsilon}{2}
$$


Assim, para todo $n \geq n_{0}$, segue de (1) e (2) que:

$$
|| \mathrm{T}_{\mid \mathrm{X}^{\prime \prime}}||=|| \mathrm{T}_{\mid \mathrm{X}^{\prime \prime}}+\mathrm{T}_{\mathrm{n}_{\mid \mathrm{X}^{\prime \prime}}}-\mathrm{T}_{\mathrm{n}_{\mid X^{\prime \prime}}}|| \leq|| \mathrm{T}_{\mid \mathrm{X}^{\prime \prime}}-\mathrm{T}_{\mathrm{n}_{\mid X^{\prime \prime}}}||+|| \mathrm{T}_{\mathrm{n}_{\mid X^{\prime \prime}}}|| \leq \frac{\varepsilon}{2}+\frac{\varepsilon}{2}=\varepsilon
$$

Logo $\forall \varepsilon>0, \forall X^{\prime}$ subespaço de $X$, conseguimos $X^{\prime \prime}$ subespaço de $X^{\prime}$ tal que ||$T_{\mid X^{\prime \prime}}|| \leq \varepsilon$. Assim pela Proposição 3.1.4 segue que T é estritamente singular.

\subsection{Operadores Compactos}

Proposição 3.2.1 Sejam X e Y espaços normados e $T: X \rightarrow Y$ uma aplicação linear. São equivalentes as seguintes propriedades:

a) T leva a bola unitária $B$ de X num conjunto relativamente compacto de Y,isto é, $\overline{T(B)}$ é compacto.

b) T leva os conjuntos limitados de X em conjuntos relativamente compactos de $Y$.

c) Toda seqüência limitada de pontos $x_{n} \in X$ contém uma subseqüência $\mathrm{x}_{\mathrm{n}_{\mathrm{k}}}$ tal que a seqüûncia $T\left(\mathrm{x}_{\mathrm{n}_{\mathrm{k}}}\right)$ é convergente em $Y$.

Prova. Sugerimos [8], pág.302.

Definição 3.2.2 Dizemos que uma aplicação linear $T: X \rightarrow Y$ é compacta se satisfaz as condições equivalentes citadas na proposição anterior.

Proposição 3.2.3 Sejam X e Y espaços normados e $T: X \rightarrow Y$ linear e contínua, cuja imagem tem dimensão finita. Então Té compacto.

Prova. Sugerimos [8], pág. 303.

Proposição 3.2.4 Sejam X um espaço normado, Y uma espaço de Banach e $T_{n}: X \rightarrow Y$ uma seqüência de operadores compactos tal que $T_{n} \rightarrow T$. Então Té compacto.

Prova. Sugerimos [8], pág.312. 
Proposição 3.2.5 Sejam X e Y espaços normados com dimensão infinita. Se $K: X \rightarrow Y$ é um operador compacto, então Ké estritamente singular.

Prova. Suponhamos que K não seja estritamente singular. Então, existe Z, subespaço fechado de $X$, com dimensão infinita, tal que $\mathrm{K}_{\mathrm{I}_{Z}}$ é isomorfismo sobre a imagem.

Sejam $B_{Z}$ uma bola unitária e fechada em $Z$, e $\left(x_{n}\right)_{n}$ uma seqüência em $B_{Z}$. Como $K$ é compacto, pela Proposição 3.2.1, existe $\left(x_{n_{k}}\right)_{k}$ subseqüência de $\left(x_{n}\right)_{n}$, tal que $\mathrm{K}\left(x_{n_{k}}\right)$ é convergente em $\mathrm{K}(\mathrm{Z})$.

Logo, existe $\mathrm{z} \in \mathrm{Z}$ tal que $\mathrm{K}\left(x_{n_{k}}\right) \rightarrow \mathrm{K}(\mathrm{z}) \Rightarrow \mathrm{K}_{\mathrm{IZ}}^{-1} \mathrm{~K}\left(x_{n_{k}}\right) \rightarrow \mathrm{K}_{\mathrm{IZ}}^{-1} \mathrm{~K}(\mathrm{z}) \Rightarrow\left(x_{n_{k}}\right)_{k} \rightarrow \mathrm{z}$.

Assim, mostramos que toda sequiência em $B_{Z}$ possui uma subseqüência convergente, isto é, $\mathrm{B}_{\mathrm{Z}}$ é compacto. Logo pela Proposição 1.2.21 e pelo Teorema 1.2.22 (Teorema de Riesz), segue que $\mathrm{Z}$ tem dimensão finita, o que é um absurdo.

Portanto,K é estritamente singular.

\subsection{Operadores de Fredholm}

Definição 3.3.1 Sejam X e Y espaços de Banach e $T \in L(Y, X)$ um operador com imagem fechada. Definimos o indice do núcleo e o indice de deficiência de $T$, respectivamente, por:

$$
n(T)=\operatorname{dim}(k e r(T)) \text { e } m(T)=\operatorname{dim}(X / / m T) .
$$

Definição 3.3.2 Sejam $X$ e Y espaços de Banach. Um operador $T \in L(Y, X)$ é dito semi-Fredholm superior se sua imagem ImT é fechada e $n(T)<\infty$. Um operador $T \in L(Y, X)$ é dito semi-Fredholm inferior se $m(T)<\infty$.

Definição 3.3.3 Sejam X e Y espaços de Banach e $T \in L(Y, X)$ um operador semi-Fredholm superior ou semi-Fredholm inferior. Definiremos o indice de $T$ por: $i(T)=n(T)-m(T)$. 
Definição 3.3.4 Sejam X e Y espaços de Banach e $T \in L(Y, X)$. Té chamado operador de Fredholm se $i(T)<\infty$, ou equivalentemente, se Té um operador semi-Fredholm superior e semi-Fredholm inferior.

Proposição 3.3.5 Sejam X e Y espaços de Banach e $T \in L(Y, X)$ um operador com imagem fechada para o qual $n(T)<\infty$. Seja $S: Y \rightarrow X$ um operador estritamente singular. Então $n(T+S)<\infty$.

Prova. Seja $\mathrm{Y}_{1} \subset \mathrm{Y}$ tal que $\mathrm{Y}=\mathrm{Y}_{1} \oplus \operatorname{Ker}(\mathrm{T})$. Então $\mathrm{T}_{\mid Y_{1}}$ é um isomorfismo. Suponhamos que $\operatorname{dim}(\operatorname{ker}(T+S))=\infty$, então existe um subespaço de dimensão infinita $Z$ de $Y_{1}$ sobre o qual $(\mathrm{T}+\mathrm{S})_{\left.\right|_{Z}}=0$, isto é, $S_{I_{Z}}=-\mathrm{T}_{\left.\right|_{Z}}$. Assim podemos concluir que $S_{I_{Z}}$ é um isomorfismo, o que é um absurdo pois $S$ é estritamente singular. $\log \mathrm{n}(\mathrm{T}+\mathrm{S})<\infty$. 


\section{UMA RELAÇÃO EM GX}

Neste capítulo daremos a definição de $u m I_{Y}+S$ - isomorfismo para em seguida definirmos uma importante relação no conjunto formado pelos subespaços fechados de dimensão infinita de um espaço de Banach $X$, o qual denotaremos por Gx. Terminamos este capítulo com a demonstração de um lema que será fundamental para o nosso trabalho.

No que segue $X$ será um espaço de Banach com dimensão infinita.

Definição 4.1 Seja X um espaço de Banach, e sejam $Y$ e $Z$ dois subespaços X com dimensão infinita.Nós dizemos que um operador T de Y em Z é um "IY $+S$ - isomorfismo" se é um isomorfismo da forma $I_{Y}+S$, onde $I_{Y}$ é inclusão de $Y$ em X e S um operador estritamente singular de $Y$ em X. Os subespaços Ye Z são então chamados $I_{Y}+S$ - isomorfos.

\section{Observações 4.2}

- O conjunto dos subespaços fechados de dimensão infinita, de um espaço de Banach X, será denotado por $G_{X}$.

- No que se segue um $I_{Y}+S$ - isomorfismo será chamado de "isomorfismo bom".

Proposição 4.3 Sejam $Y$ e $Z$ dois subespaços de dimensão infinita de um espaço de Banach $X$. Se T: $Y \rightarrow Z$ é um $I_{Y}+S-$ isomorfismo, então T-1 tambémé.

Prova. Por hipótese temos que $\mathrm{T}: \mathrm{Y} \rightarrow \mathrm{Z}$ é um isomorfismo bom, logo existe $\mathrm{S}: \mathrm{Y} \rightarrow \mathrm{X}$ estritamente singular tal que $\mathrm{T}=\mathrm{I}_{Y}+\mathrm{S}$.

Sendo $\mathrm{T}: \mathrm{Y} \rightarrow \mathrm{Z}$ um isomorfismo podemos considerar o operador $\mathrm{T}^{-1}: \mathrm{Z} \rightarrow \mathrm{Y}$.

Vamos mostrar que $\mathrm{T}^{-1}$ também é um isomorfismo bom.

$$
\mathrm{I}_{\mathrm{Z}}=\mathrm{TT}^{-1}=\left(\mathrm{I}_{Y}+\mathrm{S}\right) \mathrm{T}^{-1}=\mathrm{IYT}^{-1}+\mathrm{ST}^{-1}=\mathrm{T}^{-1}+\mathrm{ST}^{-1} \Rightarrow \mathrm{T}^{-1}=\mathrm{I}_{\mathrm{Z}}+\left(-\mathrm{ST}^{-1}\right)
$$

onde - ST-1 $^{-1}$, pela Proposição 3.1.6, é um operador estritamente singular. 
Assim pondo $\mathrm{S}^{\prime}=-\mathrm{ST}^{-1}$ temos que $\mathrm{T}^{-1}=\mathrm{I}_{\mathrm{Z}}+\mathrm{S}^{\prime}$, onde $\mathrm{S}^{\prime}$ é estritamente singular.

Portanto, $\mathrm{T}^{-1}$ é um isomorfismo bom.

Proposição 4.4 Sejam T e U dois isomorfismos bons. Então TU quando definido, também é um isomorfismo bom.

Prova. Consideremos os seguintes isomorfismos bons $\mathrm{T}: \mathrm{Z}^{\prime} \rightarrow W$ e $\mathrm{U}: \mathrm{Y} \rightarrow \mathrm{Z}$ onde $\mathrm{Z} \subseteq Z^{\prime}$.

Por hipótese temos que T é um isomorfismo bom, logo existe $S_{1}: Z^{\prime} \rightarrow X$ estritamente singular tal que $\mathrm{T}=\mathrm{I}_{Z^{\prime}}+\mathrm{S}_{1}$.

Por outro lado, temos que $U$ também é um isomorfismo bom, logo existe $S_{2}: Y \rightarrow X$ estritamente singular, tal que $\mathrm{U}=\mathrm{I}_{Y}+\mathrm{S}_{2}$.

Assim por (1) e (2) temos :

$$
\mathrm{TU}=\left(\mathrm{I}_{Z^{\prime}}+\mathrm{S}_{1}\right)\left(\mathrm{I}_{Y}+\mathrm{S}_{2}\right)=\mathrm{I}_{\mathrm{Z}^{\prime}} \mathrm{I}_{Y}+\mathrm{I}_{Z^{\prime}} \mathrm{S}_{2}+\mathrm{S}_{1} \mathrm{I}_{Y}+\mathrm{S}_{1} \mathrm{~S}_{2}=\mathrm{I}_{Y}+\mathrm{S}_{2}+\mathrm{S}_{1}+\mathrm{S}_{1} \mathrm{~S}_{2} .
$$

Agora, pondo $S=S_{2}+S_{1}+S_{1} S_{2}$ temos pelo Corolário 3.1.5 e Proposição 3.1.6 que $S$ é estritamente singular.

Logo $\mathrm{TU}=\mathrm{I}_{\mathrm{Y}}+\mathrm{S}$ onde $\mathrm{S}$ é estritamente singular.

Portanto, TU é um isomorfismo bom.

Definição 4.5 Seja X um espaço de Banach de dimensão infinita. Sejam Y e Z em GX. Definimos então a seguinte relação: $Z \leq Y$ se, e somente se, $Z$ é $I_{Z}+S$ - isomorfo a um subespaço de $Y$, ou seja, existe um isomorfismo bom entre $Z$ e um subespaço de $Y$. 
Lema 4.6 Seja X um espaço de Banach hereditariamente indecomponível (H.I.). Então a relação $\leq$ define um filtro em $G_{X}$, isto é, se $Y$ e $Z \in G_{X}$, então existe $W \in G_{X}$ tal que $W \leq Y$ e $W \leq Z$.

Prova. Sejam Y e Z em Gx, pelo Teorema de Mazur (Teorema 1.2.10) existem $\left(e^{\prime}{ }_{n}\right)_{n \in N}$ e $\left(e_{n}\right)_{n \in N}$ seqüências básicas de $\mathrm{Y}$ e Z, respectivamente, com constante $\mathrm{k}^{\prime}=2$.

Consideremos então os seguintes subespaços de $Y$ e $Z$, respectivamente: $Y^{\prime}=\left[\left(e^{\prime}\right)_{n \in N}\right]$ e $Z^{\prime}=\left[\left(e_{n}\right)_{n \in N}\right]$.

A partir daqui a demonstração será dividida em quatro partes.

Parte I. Sendo X Hereditariamente Indecomponível, pela Proposição 1.4.11, temos:

$$
\exists y \in Y^{\prime} \text { e } z \in Z^{\prime} \operatorname{com}|| y||=|| z||=1 \text { tais que }|| y-z|| \leq \frac{1}{32}
$$

onde $\mathrm{y}=\sum_{n=1}^{\infty} a_{n} e_{n}^{\prime} \quad$ e $z=\sum_{n=1}^{\infty} b_{n} e_{n}$.

Como y e z não são finitamente suportados, faremos o seguinte:

$\sum_{i=1}^{\infty} a_{i} e_{i}^{\prime}$ converge, logo: $\forall \varepsilon>0, \quad \exists \mathrm{n}_{2} \in \mathrm{N}: \mathrm{n} \geq \mathrm{n}_{2} \Rightarrow|| \sum_{i=1}^{\infty} a_{i} e_{i}^{\prime}-\sum_{i=1}^{n} a_{i} e_{i}^{\prime}||<\varepsilon$.

Tomando $\varepsilon=\frac{1}{64}$ temos, ||$\sum_{i=n_{3}}^{\infty} a_{i} e_{i}^{\prime}||<\frac{1}{64}$ onde $\mathrm{n}_{3}=\mathrm{n}+1$ e $\mathrm{n} \geq \mathrm{n}_{2}$.

De forma análoga temos $\sum_{i=1}^{\infty} b_{i} e_{i}$ converge , logo para $\varepsilon=\frac{1}{64}$, temos:

$\exists \mathrm{n}_{4} \in \mathrm{N}: \mathrm{n} \geq \mathrm{n}_{4} \Rightarrow|| \sum_{i=1}^{\infty} b_{i} e_{i}-\sum_{i=1}^{n} b_{i} e_{i}||<\frac{1}{64} \Rightarrow|| \sum_{i=n_{5}}^{\infty} b_{i} e_{i}||<\frac{1}{64}$ onde $\mathrm{n}_{5}=\mathrm{n}+1$.

Agora considerando $n_{1}=\max \left\{n_{3}, n_{5}\right\}$ temos :

$$
|| \sum_{i=n_{1}}^{\infty} a_{i} e_{i}||<\frac{1}{64} \text { e }|| \sum_{i=n_{1}}^{\infty} b_{i} e_{i}||<\frac{1}{64} \text {. }
$$


Denotando por $\mathrm{r}_{1}=\sum_{i=n_{1}}^{\infty} a_{i} e_{i}^{\prime}, \mathrm{r}_{2}=\sum_{i=n_{1}}^{\infty} b_{i} e_{i}$ e considerando os vetores $y_{0}^{\prime}=\mathrm{y}-\mathrm{r}_{1}$ e $\mathrm{z}_{0}=\mathrm{z}-\mathrm{r}_{2}$ temos :

a) $y_{0}^{\prime}=\sum_{i=1}^{n_{0}} a_{i} e_{i}^{\prime}$ e $\mathrm{z}_{0}=\sum_{i=1}^{n_{0}} b_{i} e_{i}$ onde $\mathrm{n}_{0}=\mathrm{n}_{1}-1$, logo possuem suporte finito.

Agora, utilizando (1) e (2) temos:

b) ||$y_{0}^{\prime}-z_{0}||=||\left(y-r_{1}\right)-\left(z-r_{2}\right)|| \leq|| y-z||+|| r_{2}-r_{1}|| \leq \frac{1}{32}+|| r_{2}-r_{1}||$

$\leq \frac{1}{32}+|| \mathrm{r}_{1}||+|| \mathrm{r}_{2}||<\frac{1}{32}+\frac{1}{64}+\frac{1}{64}=\frac{1}{16}$.

Assim conseguimos $y_{0}^{\prime} \in Y^{\prime}$ e $z_{0} \in Z^{\prime}$ finitamente suportados e tais que ||$y_{0}^{\prime}-z_{0}||<\frac{1}{16}$.

Notemos ainda que: ||$y_{0}^{\prime}|| \cong 1$ e ||$z_{0}|| \cong 1$.

De fato, $y_{0}^{\prime}=\mathrm{y}-\mathrm{r}_{1} \Rightarrow|| y_{0}^{\prime}||=|| \mathrm{y}-\mathrm{r}_{1}|| \leq|| \mathrm{y}||+|| \mathrm{r}_{1}||<1+\frac{1}{64}$.

Por outro lado, como $\mathrm{y}=y_{0}^{\prime}+\mathrm{r}_{1}$ temos:

$$
1=|| y||=|| y_{0}^{\prime}+r_{1}|| \leq|| y_{0}^{\prime}||+|| r_{1}||<|| y_{0}^{\prime}||+\frac{1}{64} \Rightarrow|| y_{0}^{\prime}||>1-\frac{1}{64} \text {. }
$$

Assim, $1-\frac{1}{64}<|| y_{0}^{\prime}||<1+\frac{1}{64}$ e de forma análoga temos ||$z_{0}|| \cong 1$.

Parte II. Consideremos agora $Y_{1}$ (respectivamente $Z_{1}$ ) subespaço de $Y^{\prime}$ ( respectivamente $Z^{\prime}$ ), constituído por elementos da forma $\sum_{i=n_{1}}^{\infty} a_{i} e_{i}^{j}$ (respectivamente $\sum_{i=n_{1}}^{\infty} b_{i} e_{i}$ ).

Sendo $\left\{\left(\mathrm{e}_{n}^{\prime}\right)_{\mathrm{n} \in \mathrm{N}}\right\}$ base de $\mathrm{Y}^{\prime}$ segue que $\left\{\left(e_{n}^{\prime}\right)_{n=n_{1}}^{\infty}\right\}$ é uma base de $Y_{1}, \operatorname{logo} \operatorname{dim} Y_{1}=\infty$, e por definição segue que $Y_{1}$ é um subespaço fechado de $Y^{\prime}$.

As afirmações anteriores também são válidas para o subespaço $Z_{1}$ de $Z^{\prime}$. 
Daí, utilizando o mesmo argumento da parte $I$, encontraremos vetores $y_{1}^{\prime} \in Y_{1}$ e $\mathrm{Z}_{1} \in \mathrm{Z}_{1}$, de suporte finito, tais que :

$$
|| y_{1}^{\prime}-z_{1}||<\frac{1}{32}, 1-\frac{1}{128}<|| y_{1}^{\prime}||<1+\frac{1}{128} \text { e } 1-\frac{1}{128}<|| z_{1}||<1+\frac{1}{128} \text {. }
$$

Repetindo esse procedimento iteradamente, teremos duas seqüências de base de blocos: $\left(y_{n}^{\prime}\right)_{\mathrm{n}}$ em $Y$ e $\left(z_{n}\right)_{n}$ em $Z$, e portanto pela Proposição 1.2.15, duas seqüências básicas de constante $\mathrm{k} \leq 2$.

Essas duas seqüências básicas são tais que ||$y_{n}^{\prime}-\mathrm{z}_{\mathrm{n}}||<\frac{1}{16} \cdot \frac{1}{2^{n}}, \forall \mathrm{n} \in \mathbf{N}$.

Pela Proposição 1.2.18, temos que $\left\{\frac{y_{n}^{\prime}}{\left\|y_{n}^{\prime}\right\|}\right\}_{n}$ é seqüência básica de $Y$ com a mesma constante básica de $\left(y_{n}^{\prime}\right)_{\mathrm{n}}$. E ainda temos:

$$
\begin{aligned}
& \left\|\frac{y_{n}^{\prime}}{\left\|y_{n}^{\prime}\right\|}-z_{n}\right\|=\left\|\frac{y_{n}^{\prime}}{\left\|y_{n}^{\prime}\right\|}-z_{n}+y_{n}^{\prime}-y_{n}^{\prime}\right\| \leq|| y_{n}^{\prime}-z_{n}||+\left\|\frac{y_{n}^{\prime}}{\left\|y_{n}^{\prime}\right\|}-y_{n}^{\prime}\right\|<\frac{1}{16} \cdot \frac{1}{2^{n}}+|| y_{n}^{\prime}|| \cdot\left|\frac{1}{\left\|y_{n}^{\prime}\right\|}-1\right| \\
& =\frac{1}{16} \cdot \frac{1}{2^{n}}+|| y_{n}^{\prime}|| \frac{\left|1-\left\|y_{n}^{\prime}\right\|\right|}{\left\|y_{n}^{\prime}\right\|}=\frac{1}{16} \cdot \frac{1}{2^{n}}+|1-|\left|y_{n}^{\prime}\right|||
\end{aligned}
$$

Agora lembrando, que por construção temos $1-\varepsilon_{n}<|| y_{n}^{\prime}||<1+\varepsilon_{n}$ onde $\varepsilon_{n}=\frac{1}{2^{n+6}}$ $(\operatorname{ver}(3))$, segue que : $|1-|\left|y_{n}^{\prime}\right|||<\varepsilon_{\mathrm{n}} \quad \forall \mathrm{n} \in \mathrm{N}$.

Assim, de (6) e (7) resulta que, $\left\|\frac{y_{n}^{\prime}}{\left\|y_{n}^{\prime}\right\|}-z_{n}\right\|<\frac{1}{16} \cdot \frac{1}{2^{n}}+\frac{1}{2^{n+6}}, \forall \mathrm{n} \in \mathrm{N}$.

Logo, por (4), temos:

$$
\sum_{n=1}^{\infty}\left\|\frac{y_{n}^{\prime}}{\left\|y_{n}^{\prime}\right\|}-z_{n}\right\|<\sum_{n=1}^{\infty}\left(\frac{1}{16} \cdot \frac{1}{2^{n}}+\frac{1}{2^{n+6}}\right)=\frac{1}{16}+\frac{1}{64}=\frac{5}{64}<\frac{1}{4} \leq \frac{1}{2 k} .
$$

Daí, colocando $\mathrm{y}_{\mathrm{n}}=\frac{y_{n}^{\prime}}{\left\|y_{n}^{\prime}\right\|}$ temos, $\sum_{n=1}^{\infty}|| \mathrm{y}_{\mathrm{n}}-\mathrm{z}_{\mathrm{n}}||<\frac{1}{2 k}$,

onde $\left(\mathrm{y}_{\mathrm{n}}\right)_{\mathrm{n}}$ é uma seqüência básica normalizada de $Y$ e portanto de $X$. 
Logo pela Proposição. 1.2.13, segue que $\left(\mathrm{z}_{\mathrm{n}}\right)_{\mathrm{n}}$ é seqüência básica de $X$, equivalente a $\left(y_{n}\right)_{n}$.

Parte III. Consideremos agora os seguintes subespaços de $X: Y^{\prime \prime}=\left[\left(y_{n}\right)_{n}\right]$ e $Z^{\prime \prime}=\left[\left(z_{n}\right)_{n}\right]$, segue de (10) e da Proposição 1.2.12, que existe $\mathrm{T}: \mathrm{Y}^{\prime \prime} \rightarrow \mathrm{Z}^{\prime \prime}$, isomorfismo tal que $\mathrm{T}\left(\mathrm{y}_{\mathfrak{n}}\right)=\mathrm{z}_{\mathrm{n}}$ onde $\mathrm{T}$ é definida da seguinte forma :

$$
T\left(\sum_{n=1}^{\infty} a_{n} y_{n}\right)=\sum_{n=1}^{\infty} a_{n} z_{n}
$$

Vamos agora mostrar que $\mathrm{T}=\mathrm{I}_{\gamma^{\prime \prime}}+\mathrm{K}$ onde $\mathrm{I}_{\gamma^{\prime \prime}}$ é a inclusão $\mathrm{Y}^{\prime \prime}$ em $\mathrm{X}$, e $\mathrm{K}$ um operador compacto.

$$
T\left(\sum_{n=1}^{\infty} a_{n} y_{n}\right)=\sum_{n=1}^{\infty} a_{n} z_{n}+\sum_{n=1}^{\infty} a_{n} y_{n}-\sum_{n=1}^{\infty} a_{n} y_{n}=\sum_{n=1}^{\infty} a_{n} y_{n}+\sum_{n=1}^{\infty} a_{n}\left(z_{n}-y_{n}\right) .
$$

Definindo, $\mathrm{K}: \mathrm{Y}^{\prime \prime} \rightarrow \mathrm{X}$ por $\mathrm{K}\left(\sum_{n=1}^{\infty} a_{n} y_{n}\right)=\sum_{n=1}^{\infty} a_{n}\left(z_{n}-y_{n}\right)$, temos $\mathrm{T}=\mathrm{I}_{\mathrm{Y}^{\prime \prime}}+\mathrm{K}$.

Vamos então provar as seguintes afirmações a respeito do operador K:
a) Kestá bem definido;
b) K é linear;
c) Ké contínuo;
d) Ké compacto.

Prova de (a): K está bem definida, uma vez que a série $\sum_{n=1}^{\infty} a_{n}\left(z_{n}-y_{n}\right)$ converge, pois as séries $\sum_{n=1}^{\infty} a_{n} y_{n}$ e $\sum_{n=1}^{\infty} a_{n} z_{n}$ são convergentes, e como $X$ é um espaço de Banach, segue que $\sum_{n=1}^{\infty} a_{n}\left(z_{n}-y_{n}\right) \in \mathrm{X}$

Prova de (b): Ké linear. De fato, $\forall \mathrm{x}, \mathrm{y} \in \mathrm{Y}^{\prime \prime}, \mathrm{x}=\sum_{n=1}^{\infty} a_{n} y_{n}, \mathrm{y}=\sum_{n=1}^{\infty} b_{n} y_{n}, \mathrm{e} \forall \lambda \in \mathrm{IK}$ temos :

$$
\begin{aligned}
& \mathrm{K}(\mathrm{x}+\lambda \mathrm{y})=\mathrm{K}\left(\sum_{n=1}^{\infty} a_{n} y_{n}+\lambda \sum_{n=1}^{\infty} b_{n} y_{n}\right)=\mathrm{K}\left(\sum_{n=1}^{\infty}\left(a_{n}+\lambda b_{n}\right) y_{n}\right)=\sum_{n=1}^{\infty}\left(a_{n}+\lambda b_{n}\right)\left(z_{n}-y_{n}\right)= \\
& \sum_{n=1}^{\infty} a_{n}\left(z_{n}-y_{n}\right)+\lambda \sum_{n=1}^{\infty} b_{n}\left(z_{n}-y_{n}\right)=\mathrm{K}(\mathrm{x})+\lambda \mathrm{K}(\mathrm{y}) .
\end{aligned}
$$


Prova de (c): K é contínuo. De fato, pois sendo k a constante básica, segue da Proposição 1.2.17 e de (9) que:

$$
\begin{aligned}
& || \mathrm{K}\left(\sum_{n=1}^{\infty} a_{n} y_{n}\right)||=|| \sum_{n=1}^{\infty} a_{n}\left(z_{n}-y_{n}\right)|| \leq \sum_{n=1}^{\infty}\left|a_{n}\right|\left(\left\|z_{n}-y_{n}\right\|\right) \leq 2 \mathrm{k}|| \mathrm{y}|| \sum_{n=1}^{\infty}\left(\left\|z_{n}-y_{n}\right\|\right)< \\
& \mathrm{k}|| \mathrm{y}|| \frac{1}{2 k}=|| \mathrm{y}|| \text { onde } \mathrm{y}=\sum_{n=1}^{\infty} a_{n} y_{n} \Rightarrow|| \mathrm{K}(\mathrm{y})|| \leq|| \mathrm{y}|| .
\end{aligned}
$$

Prova de (d): Para provarmos que K é compacto, iremos considerar a seguinte seqüência de operadores:

$$
\left\{\mathrm{K}_{\mathrm{p}}\right\}_{\mathrm{p} \in \mathrm{N}} \text { onde } \mathrm{K}_{\mathrm{p}}: \mathrm{Y}^{\prime \prime} \rightarrow \mathrm{X} \quad \text { e } \mathrm{K}_{\mathrm{p}}\left(\sum_{n=1}^{\infty} a_{n} y_{n}\right)=\sum_{n=1}^{p} a_{n}\left(z_{n}-y_{n}\right)
$$

A respeito desses operadores provaremos as seguintes afirmações, $\forall \mathrm{p} \in \mathrm{N}$ :

i) $\quad K_{p}$ está bem definida;

ii) $K_{p}$ é linear;

iii) $\mathrm{K}_{\mathrm{p}}$ é contínua;

iv) $K_{p}$ é compacta;

v) $\quad \mathrm{K}_{\mathrm{p}} \rightarrow \mathrm{K}$.

Prova de (i): é imediato.

Prova de (ii): demonstração análoga a prova de (b).

Prova de (iii) : Sendo y $=\sum_{n=1}^{\infty} a_{n} y_{n}$ e k a constante básica, segue da Proposição 1.2.17 e de (8) que: || $\mathrm{K}_{\mathrm{p}}(\mathrm{y})||=|| \sum_{n=1}^{p} a_{n}\left(z_{n}-y_{n}\right)|| \leq \sum_{n=1}^{p}\left|a_{n}\right|\left(\left\|z_{n}-y_{n}\right\|\right) \leq 2 \mathrm{k}|| \mathrm{y}|| \cdot \sum_{n=1}^{p}\left(\left\|z_{n}-y_{n}\right\|\right)<$ $2 \mathrm{k}|| \mathrm{y}|| \sum_{n=1}^{p}\left(\frac{1}{16} \cdot \frac{1}{2^{n}}+\frac{1}{2^{n+6}}\right)=2 \mathrm{k}|| \mathrm{y}|| \cdot\left(\frac{1}{16} \sum_{n=1}^{p}\left(\frac{1}{2^{n}}\right)+\frac{1}{64} \sum_{n=1}^{p}\left(\frac{1}{2^{n}}\right)\right)$. Agora, como para cada $\mathrm{p} \in \mathbb{N}, \sum_{n=1}^{p}\left(\frac{1}{2^{n}}\right)<1$, segue que, ||$K_{\mathrm{p}}(\mathrm{y})|| \leq \frac{5 k}{32}|| \mathrm{y}||$. Portanto, $K_{p}$ é contínuo. 
Prova de (iv) : Seja $\mathrm{y} \in \operatorname{Im}\left(\mathrm{K}_{\mathrm{p}}\right)$, então $\mathrm{y}=\sum_{n=1}^{p} a_{n}\left(z_{n}-y_{n}\right)$. Logo a dimensão da imagem de $K_{p}$ é finita $(\leq p)$. Assim pela Proposição 3.2.3 seque que $K_{p}$ é compacto.

Prova de (v): Vamos agora mostrar que $\mathrm{K}_{\mathrm{p}} \rightarrow \mathrm{K}$. Dado $\mathrm{y}=\sum_{n=1}^{\infty} a_{n} y_{n}$ temos:

|| $\mathrm{K}(\mathrm{y})-\mathrm{K}_{\mathrm{p}}(\mathrm{y})||=|| \sum_{n=1}^{\infty} a_{n}\left(z_{n}-y_{n}\right)-\sum_{n=1}^{p} a_{n}\left(z_{n}-y_{n}\right)||=|| \sum_{n=p+1}^{\infty} a_{n}\left(z_{n}-y_{n}\right)|| \leq$ $\sum_{n=p+1}^{\infty}\left|a_{n}\right|\left(\left\|z_{n}-y_{n}\right\|\right) \leq 2|| \mathrm{y}|| \mathrm{k} \sum_{n=p+1}^{\infty}\left\|z_{n}-y_{n}\right\|<2 \mathrm{k}|| \mathrm{y}|| \sum_{n=p+1}^{\infty}\left(\frac{1}{16} \cdot \frac{1}{2^{n}}+\frac{1}{2^{n+6}}\right), \forall \mathrm{p} \in \mathrm{N}$.

Logo, $\left|\mathrm{K}(\mathrm{y})-\mathrm{K}_{\mathrm{p}}(\mathrm{y})\right||\leq 2 \mathrm{k}||\mathrm{y}| \mid \sum_{n=p+1}^{\infty}\left(\frac{1}{16} \cdot \frac{1}{2^{n}}+\frac{1}{2^{n+6}}\right) \rightarrow 0$, quando $\mathrm{p} \rightarrow \infty$.

Assim, ||$K(y)-K_{p}(y)|| \rightarrow 0$, quando $\mathrm{p} \rightarrow \infty$. Por tanto, $K_{p} \rightarrow K$.

Estamos agora com a seguinte situação: $K_{p} \in L\left(Y^{\prime \prime}, X\right)$ é uma seqüência de operadores compactos e $\mathrm{K} \in \mathrm{L}\left(\mathrm{Y}^{\prime \prime}, \mathrm{X}\right)$ é tal que $\mathrm{K}_{\mathrm{p}} \rightarrow \mathrm{K}$. Assim pela Proposição 3.2.4, segue que $\mathrm{K}$ é um operador compacto, o que prova a afirmação (d) .

Parte IV. Agora estamos prontos para finalizar a demonstração do nosso lema.

Pela parte III vimos que existe $\mathrm{T}: \mathrm{Y}^{\prime \prime} \rightarrow \mathrm{Z}^{\prime \prime}$, isomorfismo da forma $\mathrm{T}=\mathrm{I}_{Y^{\prime \prime}}+\mathrm{K}$, onde $\mathrm{K}$ é um operador compacto. Mas pela Proposição 3.2.5 temos que K compacto, implica K estritamente singular.

Logo T é um "isomorfismo bom" entre $Y^{\prime \prime}$ e $Z^{\prime \prime}$ ( subespaço de $Z$ ). Assim $Y^{\prime \prime} \leq Z$.

Agora como $Y^{\prime \prime} \subset Y$ temos que $Y^{\prime \prime} \leq Y$, pois $T^{\prime}: Y^{\prime \prime} \rightarrow Y^{\prime \prime}$ dada por $T^{\prime}=I_{Y^{\prime \prime}}+0$, onde 0 é o operador nulo, é um isomorfismo bom (já que o operador nulo é estritamente singular).

Finalmente, pondo $W=Y^{\prime \prime}$ temos: $W \leq Z$ e $W \leq Y$, o que prova nosso lema. 


\section{UMA SEMI-NORMA EM L(Y,X)}

Neste capítulo apresentaremos uma semi-norma no espaço $L(Y, X)$ e demonstraremos alguns resultados relativos a essa semi-norma.

No que segue, todos os subespaços considerados serão fechados e de dimensão infinita.

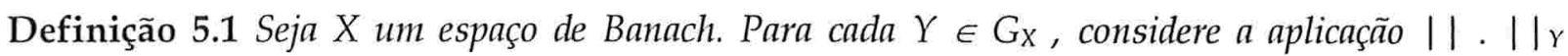
definida sobre $L(Y, X)$ por : $\|T\|_{Y}=\sup _{Z \subset Y} \inf _{Z^{\prime} \subset Z}\left\|T_{\left.\right|^{\prime}}\right\|$.

Observação 5.2|| . ||$_{y}$ está bem definida pois o conjunto $\left\{|| T_{\mid Z^{\prime}}|| / Z^{\prime} \subset Z\right\}$ é não vazio e limitado inferiormente por 0 , pois ||$T_{\mid Z^{\prime}}|| \geq 0$, logo possui ínfimo; e o conjunto $\left\{\inf _{Z^{\prime}} Z_{Z}|| T_{\mid Z^{\prime}}|| / Z \subset Y\right\}$ é não vazio e limitado superiormente por ||$T||$, logo possui supremo.

Proposição 5.3 Seja X um espaço de Banach e $Y \in G \times$. Então temos:

a) ||$T||_{\gamma}=0$ se, e somente se, Té estritamente singular.

b) A aplicação || . $\mid \gamma: L(Y, X) \rightarrow R_{+}$é uma semi-norma em $L(Y, X)$.

Prova. (a) Utilizando a definição de ínfimo, segue da Proposição 3.1.4 que:

|| $\mathrm{T}||_{Y}=0 \Leftrightarrow \inf _{Z^{\prime} \subset Z}|| T_{\mid Z^{\prime}}||=0, \forall Z \subset Y \Leftrightarrow \forall \varepsilon>0, \forall Z \subset Y, \exists Z^{\prime} \subset Z:|| T_{\mid Z^{\prime}}||<\varepsilon \Leftrightarrow$ T é estritamente singular.

Parte (b) Vamos agora mostrar que a aplicação | | | | y é um semi-norma em L(Y,X). Para tanto devemos provar que || | | | y satisfaz as seguintes propriedades:

i) || $\mathrm{T}+\mathrm{U}||_{Y} \leq|| \mathrm{T}||_{Y}+|| \mathrm{U}||_{Y} \quad \forall \mathrm{T}, \mathrm{U} \in \mathrm{L}(\mathrm{Y}, \mathrm{X})$.

ii) ||$\lambda \mathrm{T}||_{Y}=|\lambda| \cdot|| \mathrm{T}||_{Y} \quad \forall \mathrm{T} \in \mathrm{L}(\mathrm{Y}, \mathrm{X}), \forall \lambda \in \mathrm{IK}$. 
Prova de (i) : Sejam $T, U \in L(Y, X)$ e $\varepsilon>0$.

Por definição $\|T+U\|_{Y}=\sup _{Z_{\subset} Y_{Z^{\prime} \subset Z}} \inf _{\|}\left\|(T+U)_{\mid Z^{\prime}}\right\|$.

Assim, dado $\varepsilon>0, \exists Z_{o} \subset Y$ tal que $\inf _{Z^{\prime} \subset Z_{0}}\left\|(T+U)_{\mid Z^{\prime}}\right\|>\|T+U\|_{Y}-\varepsilon$, daí segue que:

||$(T+U)_{\mid Z^{\prime}}||>|| T+U|| Y-\varepsilon, \forall Z^{\prime} \subset Z_{0} \Rightarrow|| T+\left.U\right|_{Y}<||(T+U)_{Z_{Z^{\prime}}}||+\varepsilon, \forall Z^{\prime} \subset Z_{0}$.

Agora, como ||.|| é uma norma em $\mathrm{L}(\mathrm{Y}, \mathrm{X})$ temos :

$$
|| \mathrm{T}+\mathrm{U}||_{Y}<|| \mathrm{T}_{Z_{Z^{\prime}}}||+|| \mathrm{U}_{\left.\right|_{Z^{\prime}}}||+\varepsilon \quad \forall Z^{\prime} \subset Z_{0},
$$

e pondo $Z=Z^{\prime}$ segue, ||$T+\left.U\right|_{Y}<|| T_{\left.\right|_{Z}}||+|| U_{\mid Z}||+\varepsilon \quad \forall Z \subset Z_{0}$.

Olhemos agora para $\inf _{Z \subset Z_{0}}\left\|T_{\left.\right|_{Z}}\right\|$, pela definição de ínfimo:

$$
\exists Z_{1} \subset Z_{0} \text { tal que }\left\|T_{Z_{1}}\right\|<\inf _{Z \subset Z_{0}}\left\|T_{I_{Z}}\right\|+\varepsilon,
$$

e lembrando que $\forall Z \subset Z_{1}$ temos || $\mathrm{T}_{\mid Z}|| \leq|| \mathrm{T}_{\mid Z_{1}}||$ segue que:

$$
\left\|T_{I_{Z}}\right\|<\inf _{Z \subset Z_{0}}\left\|T_{I_{Z}}\right\|+\varepsilon \quad \forall Z \subset Z_{1}
$$

Assim, sendo $\mathrm{Z} \subset \mathrm{Z}_{1} \subset \mathrm{Z}_{0}$, por (2) e (3) temos:

$$
\begin{aligned}
& \|\mathrm{T}+\mathrm{U}\|_{Y}<\inf _{\mathrm{Z} \subset Z_{0}}\left\|\mathrm{~T}_{\mathrm{IZ}}\right\|+\left\|\mathrm{U}_{\mathrm{IZ}}\right\|+2 \varepsilon \Rightarrow\|\mathrm{T}+\mathrm{U}\|_{\mathrm{Y}}<\|\mathrm{T}\|_{\mathrm{Y}}+\left\|\mathrm{U}_{\mathrm{IZ}}\right\|+2 \varepsilon \Rightarrow|| \mathrm{U}_{\mathrm{IZ}}||> \\
& || \mathrm{T}+\mathrm{U}||_{Y}-|\mathrm{T}| \mid \mathrm{Y}-\varepsilon, \forall \varepsilon>0, \forall \mathrm{Z} \subset \mathrm{Z}_{1} .
\end{aligned}
$$

E utilizando novamente a definição de ínfimo, segue que :

$$
\inf _{Z \subset Z_{1}}\left\|U_{\mid Z}\right\| \geq\|T+U\|_{Y}-\|T\|_{Y}-2 \varepsilon \Rightarrow\|T+U\|_{Y} \leq \inf _{Z \subset Z_{1}}\left\|U_{\mid Z}\right\|+\|T\|_{Y}+2 \varepsilon
$$

o que implica ||$T+U||_{Y} \leq|| T||_{Y}+\left.|| U\right|_{Y}+2 \varepsilon \quad \forall \varepsilon>0$. 
Como \& é arbitrário temos, || $\mathrm{T}+\mathrm{U}||_{Y} \leq|| \mathrm{T}||_{Y}+|| \mathrm{U}||_{Y}$.

Prova de (ii): Sejam $T \in L(Y, X)$ e $\lambda \in I K$.

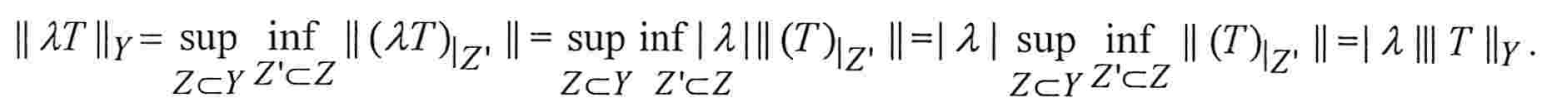

Assim, por (i) e (ii) temos que ||.||Y é um semi-norma em $L(Y, X)$.

Verifiquemos agora, que adicionando a hipótese de que $X$ é um espaço de Banach Hereditariamente Indecomponível, a semi-norma ||$. \mid \|_{Y}$ ganhará uma propriedade que irá nos ajudar muito na obtenção dos próximos resultados. Para a demonstração da próxima proposição iremos utilizar o Lema 4.6 .

Proposição 5.4 Seja X um espaço de Banach Hereditariamente Indecomponivel (H.I.). Sejam $Y \in G_{X}$ e $T \in L(Y, X)$, então a quantidade $\inf _{Z^{\prime} \subset Z}\left\|T_{Z_{Z^{\prime}}}\right\|$ não depende da escolha do subespaço $Z$ de $Y$.

Prova. Sejam $Z_{1}$ e $Z_{2}$ dois subespaços de $Y$. Vamos mostrar que sendo $\alpha=\inf _{Z^{\prime} \subset Z_{1}}\left\|T_{Z_{Z^{\prime}}}\right\|$ e $\beta=\inf _{Z^{\prime} \subset Z_{2}}\left\|T_{Z^{\prime}}\right\|$ então $\alpha=\beta$. Para tanto é suficiente provar que para todo $\varepsilon>0$ temos:

a) Dado $Z_{2}^{\prime} \subset Z_{2}, \exists Z_{1}^{\prime \prime} \subset Z_{1}$ tal que $\left\|T_{Z_{1}^{\prime \prime}}\right\| \leq\left\|T_{Z_{2}^{\prime}}\right\|+\varepsilon$.

b) Dado $Z_{1}^{\prime} \subset Z_{1}, \exists Z_{2}^{\prime \prime} \subset Z_{2}$ tal que $\left\|T_{Z_{2}^{\prime \prime}}\right\| \leq\left\|T_{Z_{1}^{\prime}}\right\|+\varepsilon$.

De fato, suponha que $\alpha>\beta$, então $\exists Z_{2}^{\prime} \subset Z_{2}$ tal que $\beta<\left\|T_{I_{2}^{\prime}}\right\|<\alpha$.

Agora, dado $\varepsilon>0$, suficientemente pequeno, teremos : $\left\|T_{Z_{2}^{\prime}}\right\|+\varepsilon<\alpha$, mas por (a) $\exists Z_{1}^{\prime \prime} \subset Z_{1}$ tal que $\left\|T_{Z_{1}^{\prime \prime}}\right\| \leq\left\|T_{Z_{2}^{\prime}}\right\|+\varepsilon \Rightarrow\left\|T_{Z_{1}^{\prime \prime}}\right\|<\alpha$, o que é um absurdo, pois $\alpha=\inf _{Z^{\prime} \subset Z_{1}}\left\|T_{Z^{\prime}}\right\|$. Logo $\alpha \leq \beta$.

Mostraremos agora que não pode ocorrer $\alpha<\beta$. 
De fato, suponha que $\alpha<\beta$, então $\exists Z_{1}{ }^{\prime} \subset Z_{1}$ tal que $\alpha<\left\|T_{Z_{1}{ }^{\prime}}\right\|<\beta$, e assim para algum $\varepsilon>0$, suficientemente pequeno, teremos : $\left\|T_{Z_{1}^{\prime}}\right\|+\varepsilon<\beta$. Mas por (b) segue que $\exists Z_{2}^{\prime \prime} \subset Z_{2}$ tal que $\left\|T_{Z_{2}^{\prime \prime}}\right\| \leq\left\|T_{Z_{1}^{\prime}}\right\|+\varepsilon \Rightarrow\left\|T_{Z_{2}^{\prime \prime}}\right\|<\beta$, o que é um absurdo, pois $\beta=\inf _{Z^{\prime} \subset Z_{2}}\left\|T_{Z_{Z^{\prime}}}\right\|$.

Logo, só nos resta uma única possibilidade, que é $\alpha=\beta$.

Vamos então provar a afirmação (a).

Sendo $Z_{1}$ e $Z_{2}$ dois subespaços de $Y$, dado $\varepsilon^{\prime}>0$ e $Z_{2}^{\prime} \subset Z_{2}$, utilizando o Lema 4.6, segue que existe um isomorfismo bom entre um subespaço $W$ de $Z_{2}^{\prime}$ e um subespaço $Z_{1}^{\prime}$ de $Z_{1}$, o qual denotaremos por $I_{w}+S$, onde $S$ é um operador estritamente singular de $W$ em $X$ e IW é a inclusão de $W$ em $X$, isto é: $I_{W}+S: W \rightarrow Z_{1}{ }^{\prime}$.

Agora, passando para subespaços, podemos assumir que ||$\left(\mathrm{I}_{W}+\mathrm{S}\right)^{-1}|| \leq 1+\varepsilon^{\prime}$.

De fato, como $S$ é estritamente singular, pela Proposição 3.1.4, dado $\varepsilon^{\prime}>0$, conseguimos $W^{\prime}$ subespaço de $W$ tal que:

$$
\left|\mathrm{S}_{\mid W^{\prime}}\right| \mid \leq \frac{\varepsilon^{\prime}}{k}, \text { onde } \mathrm{k}=||\left(\mathrm{I}_{W}+S\right)^{-1}||,
$$

e segue da Proposição 4.3 que : $\left(I_{W}+S\right)^{-1}=I_{Z_{1}^{\prime}}-S\left(I_{W}+S\right)^{-1}$.

Agora sendo $Z_{1}{ }^{\prime \prime}=\left(I_{W}+S\right)\left(W^{\prime}\right)$ e restringindo $\left(I_{W}+S\right)^{-1}$ ao subespaço $Z_{1}{ }^{\prime \prime}$ de $Z_{1}$ temos :

$$
\left\|\left.\left(I_{W}+S\right)^{-1}\right|_{Z_{1}^{\prime \prime}}\right\|=\left.||\left(I_{Z_{1}^{\prime}}-S\left(I_{W}+S\right)^{-1}\right)_{Z_{1}^{\prime \prime}}\|\leq\| I_{Z_{1}^{\prime \prime}}\|+\| S_{W_{W^{\prime}}}\|\cdot\|\left(I_{W}+S\right)^{-1}\right|_{Z_{1}^{\prime \prime}} \| .
$$

Como $Z_{1}^{\prime \prime} \subset Z_{1}^{\prime}$, temos, $\left\|\left.\left(I_{W}+S\right)^{-1}\right|_{Z_{1}^{\prime \prime}}\right\| \leq\left\|\left(I_{W}+S\right)^{-1}\right\|$, assim

$$
\left\|\left.\left(I_{W}+S\right)^{-1}\right|_{Z_{1}}\right\| \leq\left\|I_{Z_{1}}\right\|+\left\|S_{W^{\prime}}\right\| \cdot\left\|\left(I_{W}+S\right)^{-1}\right\|,
$$

e por (2) segue que $\left\|\left.\left(I_{W}+S\right)^{-1}\right|_{Z_{1}^{\prime \prime}}\right\| \leq 1+\frac{\varepsilon^{\prime}}{k} \cdot \mathrm{k}=1+\varepsilon^{\prime}$. 
Seja agora $T \in L(Y, X)$ e, considerando a restrição de $T$ ao subespaço $Z_{1}^{\prime}$ de $Z_{1}$, e portanto subespaço de $Y$, temos :

$$
T_{Z_{1}^{\prime}}=T\left(I_{W}+S\right)\left(I_{W}+S\right)^{-1}
$$

Agora lembrando que $W$ e $Z_{1}^{\prime}$ são subespaços de $Y$ e que $I_{W}+S: W \rightarrow Z_{1}^{\prime}$ é um isomorfismo, segue que, dado $w \in W,\left(I_{W}+S\right)(w)=I_{W}(w)+S(w)$ implica $S(w) \in Y$.

Assim S toma valores em Y, e portanto, TS está bem definida. Daí podemos reescrever (3) como :

$$
T_{Z_{1}^{\prime}}=\left(T I_{W}+T S\right)\left(I_{W}+S\right)^{-1}=\left(T_{\left.\right|_{W}}+T S\right)\left(I_{W}+S\right)^{-1}
$$

e restringindo $\mathrm{T}$ ao subespaço $\mathrm{Z}_{1}^{\prime \prime}$ de $\mathrm{Z}_{1}^{\prime}$ temos :

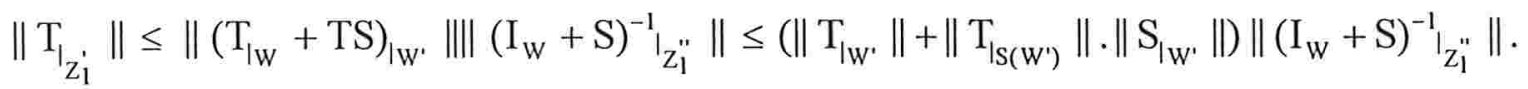

Como $W^{\prime} \subset Z_{2}^{\prime}$ e $S\left(W^{\prime}\right) \subset Y$ segue que: $\left\|T_{I_{Z_{1}^{\prime \prime}}}\right\| \leq\left(\left\|T_{I_{Z_{2}^{\prime}}}\right\|+\|T\| \cdot\left\|S_{W^{\prime}}\right\|\right) \cdot\left(I_{W}+S\right)^{-1} I_{Z_{1}^{\prime \prime}} \|$.

Agora, por (1) e (2) temos:

$\left\|\mathrm{T}_{\mathrm{I}_{1}^{\prime \prime}}\right\| \leq\left(\left\|\mathrm{T}_{\mathrm{I}_{\mathrm{z}_{2}^{\prime}}}\right\|+\|\mathrm{T}\| \cdot \frac{\varepsilon}{\mathrm{k}}\right) \cdot\left(1+\varepsilon^{\prime}\right)=\left\|\mathrm{T}_{\mathrm{I}_{\mathrm{z}_{2}^{\prime}}}\right\|+\varepsilon^{\prime} \cdot\left\|\mathrm{T}_{\mathrm{I}_{\mathrm{z}_{2}^{\prime}}}\right\|+\|\mathrm{T}\| \cdot \frac{\varepsilon^{\prime}}{\mathrm{k}} \cdot\left(1+\varepsilon^{\prime}\right) \leq$

$\left\|\mathrm{T}_{\mathrm{I}_{\mathrm{z}_{2}}}\right\|+\varepsilon^{\prime} \cdot\|\mathrm{T}\|+\|\mathrm{T}\| \cdot \frac{\varepsilon^{\prime}}{\mathrm{k}} \cdot\left(1+\varepsilon^{\prime}\right)$.

Agora, pondo $\varepsilon=\varepsilon^{\prime} \cdot\|T\|+\|T\| \cdot \frac{\varepsilon^{\prime}}{k} \cdot\left(1+\varepsilon^{\prime}\right)$ e sendo $\varepsilon^{\prime}>0$ arbitrário segue que:

$$
\left\|\mathrm{T}_{\mathrm{I}_{\mathrm{Z}_{1}}}\right\| \leq\left\|\mathrm{T}_{\mathrm{I}_{\mathrm{Z}_{2}^{\prime}}}\right\|+\varepsilon
$$

De forma análoga provamos a afirmação (b). 
Corolário 5.5 Seja $X$ um espaço de Banach H.I. e $Y \in G_{X}$. Então para todo $T \in L(Y, X)$ e para todo $Z \subset Y$ temos :

$$
\|T\|_{Y}=\inf _{Z^{\prime} \subset Z}\left\|T_{Z^{\prime}}\right\| .
$$

Prova. Decorre diretamente da proposição anterior.

Proposição 5.6 Seja X um espaço de Banach H.I. Sejam $Y, Z \in G X, T \in L(Y, X)$ e $U \in L(Z, Y)$. Então:

$$
|| T U|| z \leq|| T|| \gamma \cdot|| U||_{z}
$$

Prova. Vamos fazer essa demonstração em duas partes, primeira supondo que U seja estritamente singular, e segunda, supondo que U não seja estritamente singular.

Parte (a) - Se U é estritamente singular, então pela Proposição 3.1.6 temos que TU também é estritamente singular. Assim pela Proposição 5.3 segue que || $\mathrm{TU}||_{z}=0$, logo vale:

$$
|| T U|| z \leq|| T|| x \cdot|| U|| z
$$

Parte (b) - Agora, se U não é estritamente singular temos:

$$
\exists Z^{\prime} \subset Z \text { tal que } U: Z^{\prime} \rightarrow U\left(Z^{\prime}\right) \text { é um isomorfismo. }
$$

Por definição: $\|T U\|_{Z^{\prime}}=\inf _{W^{\prime} \subset W}\left\|(T U)_{\mid W^{\prime}}\right\|, \forall \mathrm{W} \subset \mathrm{Z}$, em particular $\forall \mathrm{W} \subset \mathrm{Z}^{\prime}$.

Mas ||$(\mathrm{TU})_{W_{W^{\prime}}}|| \leq|| \mathrm{T}_{\left.\right|_{U\left(W^{\prime}\right)}}|| .|| \mathrm{U}_{\left.\right|_{W^{\prime}}}|| \forall \mathrm{W}^{\prime} \subset \mathrm{W}$, e como $\mathrm{U}\left(\mathrm{W}^{\prime}\right) \subset \mathrm{U}(\mathrm{W})$ temos :

$$
||(\mathrm{TU})_{W_{W^{\prime}}}|| \leq|| \mathrm{T}_{\mid U(W)}|| \cdot|| \mathrm{U}_{\left.\right|_{W^{\prime}}}||, \forall \mathrm{W}^{\prime} \subset \mathrm{W} \subset \mathrm{Z}^{\prime} .
$$

Logo, $\inf _{W^{\prime} \subset W}\left(\left\|T_{\mid U(W)}\right\| \cdot\left\|U_{\mid W^{\prime}}\right\|\right) \geq \inf _{W^{\prime} \subset W}\left\|(T U)_{\mid W^{\prime}}\right\|$ e por (2) segue que: $\inf _{W^{\prime} \subset W}\left(\left\|T_{\mid U(W)}\right\| \cdot\left\|U_{W^{\prime}}\right\|\right) \geq\|T U\|_{Z} \forall W^{\prime} \subset W \subset Z^{\prime}$. 
Assim, $\left\|T_{\mid U(W)}\right\| \inf _{W^{\prime} \subset W}\left(\left\|U_{\mid W^{\prime}}\right\|\right) \geq\|T U\|_{Z}$

Agora, lembrando que $\inf _{W^{\prime} \subset W}\left\|U_{W^{\prime}}\right\|=\|U\|_{Z}$, temos:

$\|T U\|_{Z} \leq\|U\|_{Z}\left\|T_{\left.\right|_{U(W)}}\right\| \forall \mathrm{W} \subset \mathrm{Z}^{\prime}$.

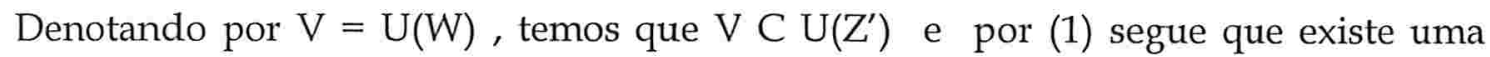
bijeção entre o conjunto dos subespaços de $Z^{\prime}$ e o conjunto dos subespaços de U(Z') logo, podemos reescrever (3) da seguinte forma: $\|T U\|_{Z} \leq\|U\|_{Z}\left\|T_{V}\right\| \forall \mathrm{V} \subset \mathrm{U}\left(\mathrm{Z}^{\prime}\right)$.

Assim, $\inf _{\mathrm{v} \subset \mathrm{U}\left(\mathrm{Z}^{\prime}\right)}\|U\|_{Z}\left\|T_{\left.\right|_{V}}\right\| \geq\|T U\|_{Z} \Rightarrow\|T U\|_{Z} \leq\|U\|_{Z} \inf _{V \subset U\left(Z^{\prime}\right)}\left\|T_{l_{V}}\right\|, \forall \mathrm{U}\left(\mathrm{Z}^{\prime}\right) \subset \mathrm{Y}$,

e finalmente, lembrando que $\inf _{V \subset U\left(Z^{\prime}\right)}\left\|T_{V}\right\|=\|T\|_{Y}$ temos: $\|T U\|_{Z} \leq\|T\|_{Y}\|U\|_{Z}$. 


\section{O ESPAÇO QUOCIENTE EY}

\subsection{O Espaço Quociente Ex}

Na presente seção daremos início a construção do espaço quociente EY e definiremos nesse espaço uma importante aplicação que será denotada por PYZ.

No que se segue, $X$ será um espaço de Banach Hereditariamente Indecomponível.

Definição 6.1.1 Para cada $Y \in G_{X}$, denotaremos por $E_{Y}$ o espaço quociente de $L(Y, X)$ pelo kernel da aplicação ||$.|| \gamma$, isto é , $\mathrm{E}_{\mathrm{Y}}=\mathrm{L}(\mathrm{Y}, \mathrm{X}) / \mathrm{S}(\mathrm{Y}, \mathrm{X})$.

\section{Observações 6.1.2}

a) Vimos na Proposição 5.3 que | | | | ré uma semi-norma no espaço $L(Y, X)$, logo segue diretamente da Proposição 1.3.5 que $S(Y, X)$ é subespaço de $L(Y, X)$.

b) Denotaremos por $\alpha_{Y}$ um elemento de $E_{Y}$, e para todo $T \in L(Y, X)$, denotaremos por $\bar{T}$ a classe de $T$ em $E_{Y}$, isto é $: \bar{T}=\{U \in L(Y, X): U-T \in S(Y, X)\}$.

c) Podemos mostrar que $E_{Y}=\{\bar{T}: T \in L(Y, X)\}$.

d) O espaço $E_{Y}$ não é necessariamente completo, pois a norma que será definida em EY não é uma norma como definida na Proposição 1.3.2.

Proposição 6.1.3 Considere a seguinte aplicação $\|\cdot\|_{Y}: E_{Y} \rightarrow \mathbf{R}_{+}$dada por $\left\|\alpha_{Y}\right\|\left\|_{Y}=\right\| T \|_{Y}$, $\forall T \in \alpha_{Y} .\|\| \cdot \|_{Y}$ está bem definida é é uma norma em $E_{Y}$.

Prova. Segue diretamente da Proposição 1.3.5 .

Definição 6.1.4 Sejam $Z, Y \in G_{X}$ tal que $Z \leq Y$. Existe $Y^{\prime}$ subespaço de $Y$ tal que $Y^{\prime}=\left(I_{Z}+S\right)(Z)$ onde $I_{Z}+S$ é um isomorfismo bom. Definimos então a seguinte aplicação de EY em EZ:

$$
\begin{aligned}
P_{Y Z}: E_{Y} & \rightarrow E_{Z} \\
\bar{T} & \rightarrow \overline{T\left(I_{Z}+S\right) .}
\end{aligned}
$$


Observação 6.1.5 Vamos agora mostrar que a aplicação PYZ está bem definida.

Parte (a) - Como $\mathrm{I}_{Z}+\mathrm{S}: \mathrm{Z} \rightarrow \mathrm{Y}^{\prime} \subset \mathrm{Y}$ e $\mathrm{T}: \mathrm{Y} \rightarrow \mathrm{X}$ temos que $\mathrm{T}\left(\mathrm{I}_{Z}+\mathrm{S}\right)$ está bem definida e $\mathrm{T}\left(\mathrm{I}_{\mathrm{Z}}+\mathrm{S}\right): \mathrm{Z} \rightarrow \mathrm{X}, \log \mathrm{O}:$

$$
\mathrm{T}\left(\mathrm{I}_{\mathrm{Z}}+\mathrm{S}\right) \in \mathrm{L}(\mathrm{Z}, \mathrm{X}) \Rightarrow \overline{T\left(I_{Z}+S\right)} \in \mathrm{E}_{\mathrm{Z}}
$$

Parte (b) - Mostraremos agora que $P_{Y Z}$ não depende da escolha dos representantes, isto é:

$$
\overline{T_{1}}=\overline{T_{2}} \Rightarrow P_{Y Z}\left(\overline{T_{1}}\right)=P_{Y Z}\left(\overline{T_{2}}\right) \text {. }
$$

Sabemos que, $\bar{T}_{1}=\bar{T}_{2} \Rightarrow T_{1} \in \bar{T}_{2} \Rightarrow T_{1}-T_{2} \in S(Y, X)$

Por definição: $P_{Y Z}\left(\overline{T_{1}}\right)=\overline{T_{1}\left(I_{Z}+S\right)}$ e $\quad P_{Y Z}\left(\overline{T_{2}}\right)=\overline{T_{2}\left(I_{Z}+S\right)}$.

Assim, para mostrarmos que $P_{Y Z}\left(\overline{T_{1}}\right)=P_{Y Z}\left(\overline{T_{2}}\right)$ é suficiente provarmos que :

$$
T_{1}\left(I_{Z}+S\right) \in \overline{T_{2}\left(I_{Z}+S\right)} \text {. }
$$

O que de fato ocorre, pois utilizando (1) e a Proposição 3.1 .6 temos:

$$
T_{1}\left(I_{Z}+S\right)-T_{2}\left(I_{Z}+S\right)=\left(T_{1}-T_{2}\right)\left(I_{Z}+S\right) \in \mathrm{S}(\mathrm{Y}, \mathrm{X})
$$

Parte (c) - Mostraremos agora, que a aplicação PYZ também não depende da escolha do operador S, isto é:

$$
P_{Y Z}\left(\overline{T_{1}}\right)=\overline{T_{1}\left(I_{Z}+S_{1}\right)} \text { e } P_{Y Z}\left(\overline{T_{1}}\right)=\overline{T_{1}\left(I_{Z}+S_{2}\right)} \Rightarrow \overline{T_{1}\left(I_{Z}+S_{1}\right)}=\overline{T_{1}\left(I_{Z}+S_{2}\right)} .
$$

O que de fato ocorre, pois:

$$
T_{1}\left(I_{Z}+S_{1}\right)-T_{1}\left(I_{Z}+S_{2}\right)=T_{1}\left(I_{Z}+S_{1}-I_{Z}-S_{2}\right)=T_{1}\left(S_{1}+\left(-S_{2}\right)\right) \in \mathrm{S}(\mathrm{Y}, \mathrm{X}) .
$$


Proposição 6.1.6 Sejam $W, Z$ e Y em $G_{X}$ tal que $W \leq Z \leq Y$. Então $P_{Y W}=P_{Z W} P_{Y Z}$.

Prova. Por hipótese temos que existem os seguintes isomorfismos:

$$
\mathrm{I}_{\mathrm{W}}+\mathrm{S}_{1}: \mathrm{W} \rightarrow \mathrm{Z}^{\prime} \subset \mathrm{Z} \quad \text { e } \quad \mathrm{I}_{\mathrm{Z}}+\mathrm{S}_{2}: \mathrm{Z} \rightarrow \mathrm{Y}^{\prime} \subset \mathrm{Y}
$$

Assim podemos definir a seguinte composição $\left(\mathrm{I}_{\mathrm{Z}}+\mathrm{S}_{2}\right)\left(\mathrm{I}_{\mathrm{W}}+\mathrm{S}_{1}\right): \mathrm{W} \rightarrow \mathrm{Y}^{\prime}$, que pela Proposição 4.4 é um isomorfismo bom entre $W$ e $Y^{\prime}$.

Logo podemos escrever, $\left(I_{Z}+S_{2}\right)\left(I_{W}+S_{1}\right)=I_{W}+S$.

Consideremos agora $\bar{T} \in E_{Y}$. Daí segue:

$\operatorname{PZW}_{\mathrm{ZYZ}}(\bar{T})=\mathrm{P}_{\mathrm{ZW}}\left(\mathrm{P}_{Y Z}(\bar{T})\right)=\mathrm{P}_{\mathrm{ZW}}\left(\overline{T\left(I_{Z}+S_{2}\right)}\right)=\overline{\left(T\left(I_{Z}+S_{2}\right)\right)\left(I_{W}+S_{1}\right)}=\overline{T\left(\left(I_{Z}+S_{2}\right)\left(I_{W}+S_{1}\right)\right)}$

$=\overline{T\left(I_{W}+S\right)}=\operatorname{PYW}(\bar{T})$.

Como $\bar{T} \in E_{Y}$ é arbitrário, temos $\mathrm{P}_{\mathrm{ZW}} \mathrm{P}_{\mathrm{YZ}}=\mathrm{P}_{Y W}$.

Lema 6.1.7 Sejam Ye Z em $G_{X}$ tal que $Z \leq Y$. Então PYZ é uma isometria linear.

Prova. Parte (a) : PYZ é linear. De fato, dados $\bar{T}_{1}, \overline{T_{2}} \in$ EY e $\lambda \in \mathrm{IK}$, temos:

$$
\begin{aligned}
& \operatorname{PYZ}\left(\overline{T_{1}}+\lambda \overline{T_{2}}\right)=\operatorname{PYZ}\left(\overline{T_{1}+\lambda T_{2}}\right)=\overline{\left(T_{1}+\lambda T_{2}\right)\left(I_{Z}+S\right)}=\overline{\left[T_{1}\left(I_{Z}+S\right)\right]+\left[\lambda T_{2}\left(I_{Z}+S\right)\right]}= \\
& \overline{\left[T_{1}\left(I_{Z}+S\right)\right]+\lambda\left[T_{2}\left(I_{Z}+S\right)\right]}=\overline{T_{1}\left(I_{Z}+S\right)}+\lambda \overline{\left[T_{2}\left(I_{Z}+S\right)\right]}=\operatorname{PYZ}\left(\overline{T_{1}}\right)+\lambda \operatorname{PYZ}\left(\overline{T_{2}}\right) .
\end{aligned}
$$


Parte (b) : Vamos agora mostrar que ||$\left|P_{Y Z}\left(\alpha_{Y}\right)\right||| z \leq||\left|\alpha_{Y}\right||| Y, \forall \alpha_{Y} \in E_{Y}$.

Seja T um representante de $\alpha_{\text {Y. }}$ Assim, pelas Proposições 6.1.3, 5.6 e 5.3 temos:

$$
\begin{aligned}
& ||\left|P_{Y Z}\left(\alpha_{Y}\right)\right||| z=||\left|P_{Y Z}(\bar{T})\right||| z=||\left|\overline{T\left(I_{Z}+S\right)}\right||| z=|| T\left(I_{Z}+S\right)|| z \leq|| T|| Y|| I_{Z}+S|| z \\
& \leq|| \mathrm{T}|| Y\left(|| I_{Z}||_{Z}+|| S|| z\right)=|| T|| Y(1+0)=|| T|| Y=||\left|\alpha_{Y}\right||| Y . \\
& \text { Logo, }||\left|P_{Y Z}\left(\alpha_{Y}\right)\right||| z \leq||\left|\alpha_{Y}\right||| Y \quad \forall \alpha_{Y} \in E_{Y}, \text { e portanto }|| P_{Y Z}|| \leq 1 .
\end{aligned}
$$

\section{Vamos agora analisar as seguintes situações:}

Parte (c) : Suponhamos que Z e Y são $\left(\mathrm{I}_{Z}+\mathrm{S}\right)$ - isomorfos, então existe um isomorfismo bom entre $\mathrm{Z}$ e Y. Mas, como a inversa de um isomorfismo bom é um isomorfismo bom (ver proposição 4.3) temos:

Existe $I_{Y}+S^{\prime}: Y \rightarrow Z$ isomorfismo bom, onde $I_{Y}+S^{\prime}=\left(I_{Z}+S\right)^{-1}$. Logo $Y \leq Z$.

Assim podemos definir a aplicação $P_{Z Y}: E_{Z} \rightarrow E_{Y}$ e de forma análoga ao que foi provado na parte $b$ teremos, $\left|P_{Z Y}\right| \mid \leq 1$.

Mas, dado $\alpha_{Y} \in E_{Y}$ e T um representante de $\alpha_{Y}$ temos:

$\mathrm{P}_{Z Y} \mathrm{P} Y Z_{Y}\left(\alpha_{Y}\right)=\mathrm{P}_{Z Y}\left(\overline{T\left(I_{Z}+S\right)}\right)=\overline{T\left(I_{Z}+S\right)\left(I_{Y}+S^{\prime}\right)}=\overline{T\left(I_{Z}+S\right)\left(I_{Z}+S\right)^{-1}}=\overline{T I_{Y}}=\bar{T}=\alpha Y$.

Logo, $P_{Z Y} P_{Y Z}\left(\alpha_{Y}\right)=\alpha_{Y}, \forall \alpha_{Y} \in E_{Y}$.

Assim, utilizando o fato de que $P_{Z Y}$ é linear e que ||$P_{Z Y}|| \leq 1$ temos:

$$
\begin{aligned}
& ||\left|\alpha_{Y}\right|||_{Y}=\left.||\left|P_{Z Y} P_{Y Z}\left(\alpha_{Y}\right)\right|||\right|_{Y}=\left.||\left|P_{Z Y}\left(P_{Y Z}\left(\alpha_{Y}\right)\right)\right|||\right|_{Y} \leq|| P_{Z Y}|| \cdot||\left|P_{Y Z}\left(\alpha_{Y}\right)\right||| Z \\
& \leq\left|P_{Y Z}\left(\alpha_{Y}\right)\right||| z .
\end{aligned}
$$




$$
||\left|\alpha_{Y}\right|||_{Y} \leq||\left|P_{Y Z}\left(\alpha_{Y}\right)\right||| Z \quad \forall \alpha_{Y} \in E_{Y} .
$$

Logo por (1) e (3) segue que ||$\left|P_{Y Z}\left(\alpha_{Y}\right)\right|||_{Z}=||\left|\alpha_{Y}\right||| Y \forall \alpha_{Y} \in E_{Y}$, ou seja, mostramos que se $Z$ e $Y$ são $\left(I_{Z}+S\right)$ - isomorfos então a aplicação linear PYZ é uma isometria.

Parte (d) : Suponhamos agora que $Z \subset$ Y . Pela Proposição 5.4 segue que:

$$
\forall \mathrm{T} \in \mathrm{L}(\mathrm{Y}, \mathrm{X}), \text { vimos que }|| \mathrm{T}||_{Y}=\inf \left\{|| T_{W_{W^{\prime}}} \|: \mathrm{W}^{\prime} \subset \mathrm{W}\right\}, \forall \mathrm{W} \subset \mathrm{Y}
$$

Restringindo $\mathrm{T}$ a um subespaço $\mathrm{Z} \subset \mathrm{Y}$ temos:

||$T|| z=\inf \left\{|| T_{W^{\prime}}||: W^{\prime} \subset W\right\}, \forall W \subset Z$ e como $Z \subset Y$ segue que ||$T|| z=|| T|| \gamma$. (5)

Agora, sendo $\mathrm{Z} \subset \mathrm{Y}$ temos que $\mathrm{Z} \leq \mathrm{Y}$ onde $\mathrm{I}_{\mathrm{Z}}+0: \mathrm{Z} \rightarrow \mathrm{Y}$ é um isomorfismo bom entre Z e um subespaço de Y. Assim dado $\alpha_{Y} \in E_{Y}$ e T um representante de $\alpha_{Y}$ temos:

$$
||\left|P_{Y Z}\left(\alpha_{Y}\right)\right||| z=||\left|\overline{T\left(I_{Z}+0\right)}\right||| z=||\left|\bar{T}_{\left.\right|_{Z}}\right||| z=|| \bar{T}||_{Y}=|| \alpha_{Y}||_{Y} .
$$

Logo, PYZ é uma isometria.

O caso geral é então conseqüência de (c) e (d) e da definição da relação $\leq$. Observe:

Parte (e) : $Z \leq Y$ implica que existe um isomorfismo bom entre $Z$ e um subespaço de $Y^{\prime}$ de $Y$.

Assim, $\mathrm{Z}$ é $\left(\mathrm{I}_{\mathrm{Z}}+\mathrm{S}\right)$ - isomorfo a $\mathrm{Y}^{\prime}$ e então pela parte (c) teremos que a aplicação

$$
P_{Y^{\prime} Z}: E_{Y^{\prime}} \rightarrow E_{Z} \text { é uma isometria. }
$$

Mas, $Y^{\prime} \leq Y$ pois $Y^{\prime} \subset Y$, logo pela parte (d) temos que a aplicação

$$
P_{Y Y^{\prime}}: E_{Y} \rightarrow E_{Y^{\prime}} \text { é uma isometria. }
$$


Agora, como $Z \leq Y^{\prime} \leq Y$ pela Proposição 6.1.6 temos : $P_{Y^{\prime} Z} P_{Y Y^{\prime}}=P_{Y Z}$.

Assim dado $\alpha_{Y} \in$ EY segue de (6) e (7) que:

$P_{Y Z}\left(\alpha_{Y}\right)=P_{Y^{\prime} Z} P_{Y Y^{\prime}}\left(\alpha_{Y}\right) \Rightarrow||\left|P_{Y Z}\left(\alpha_{Y}\right)\right||| z_{z}=||\left|P_{Y^{\prime} Z}\left(P_{Y Y^{\prime}}\left(\alpha_{Y}\right)\right)\right||| z_{z}=||\left|P_{Y Y^{\prime}}\left(\alpha_{Y}\right)\right||| z=||\left|\alpha_{Y}\right||| Y$

Logo, ||$\left|P_{Y Z}\left(\alpha_{Y}\right)\right||| z=||\left|\alpha_{Y}\right||| Y, \forall \alpha_{Y} \in E_{Y}$.

\subsection{Um produto para os elementos de $E_{Y}$}

Nesta seção daremos a definição de um produto para os elementos de EY, similar a composição de operadores lineares.

Definição 6.2.1 Sejam $Y$ e Z em $G_{X}$, denotaremos por $E_{Z Y}$ o espaço dos elementos de $E_{Z}$ que possuem um representante $T$ tal que $\operatorname{Im} T \subset Y$, istoé :

$$
E_{Z Y}=\left\{\alpha_{Z} \in E_{Z} / \exists T \in \alpha_{Z}, T: Z \rightarrow X \operatorname{com} \operatorname{Im} T \subset Y\right\}
$$

Definição 6.2.2 Sejam $Y$ e Z em $G x$, definimos então a seguinte aplicação

$$
\begin{aligned}
\otimes: E_{Y} \times E_{Z Y} & \rightarrow E_{Z} \\
(\bar{T}, \bar{U}) & \rightarrow \otimes(\bar{T}, \bar{U})=\bar{T} \otimes \bar{U}=\overline{T U}
\end{aligned}
$$

onde Té um representante em $L(Y, X)$, e U um representante em $L(Z, X)$ tal que $\operatorname{Im} U \subset Y$.

Observação 6.2.3 Vamos agora mostrar que a aplicação $\otimes$ está bem definida.

Como $U: Z \rightarrow Y \subset X$ e $\mathrm{T}: \mathrm{Y} \rightarrow \mathrm{X}$, então TU está bem definida e TU $: Z \rightarrow X$. Logo $\overline{T U} \in \mathrm{E}_{\mathrm{Z}}$

Verifiquemos agora que $\otimes$ não depende da escolha dos representantes, isto é:

$$
\bar{T}_{1}=\bar{T}_{2} \text { e } \overline{U_{1}}=\overline{U_{2}} \Rightarrow \bar{T}_{1} \otimes \overline{U_{1}}=\overline{T_{2}} \otimes \overline{U_{2}} .
$$


De fato, por hipótese temos: $\bar{T}_{1}=\bar{T}_{2} \Rightarrow \mathrm{T}_{1}-\mathrm{T}_{2} \in \mathrm{S}(\mathrm{Y}, \mathrm{X})$ e $\overline{U_{1}}=\overline{U_{2}} \Rightarrow \mathrm{U}_{1}-\mathrm{U}_{2} \in \mathrm{S}(\mathrm{Z}, \mathrm{Y})$.

Vamos então mostrar que $\overline{T_{1} U_{1}}=\overline{T_{2} U_{2}}$.

$$
\mathrm{T}_{1} \mathrm{U}_{1}-\mathrm{T}_{2} \mathrm{U}_{2}=\mathrm{T}_{1} \mathrm{U}_{1}-\mathrm{T}_{2} \mathrm{U}_{2}-\mathrm{T}_{1} \mathrm{U}_{2}+\mathrm{T}_{1} \mathrm{U}_{2}=\mathrm{T}_{1}\left(\mathrm{U}_{1}-\mathrm{U}_{2}\right)+\left(\mathrm{T}_{1}-\mathrm{T}_{2}\right) \mathrm{U}_{2}
$$

Daí pelo Corolário 3.1.5 e pela Proposição 3.1.6 segue que:

$$
\mathrm{T}_{1} \mathrm{U}_{1}-\mathrm{T}_{2} \mathrm{U}_{2} \in \mathrm{S}(\mathrm{Z}, \mathrm{X}) \Rightarrow \mathrm{T}_{1} \mathrm{U}_{1} \in \overline{T_{2} U_{2}} \Rightarrow \overline{T_{1} U_{1}}=\overline{T_{2} U_{2}} \Rightarrow \overline{T_{1}} \otimes \overline{U_{1}}=\overline{T_{2}} \otimes \overline{U_{2}} .
$$

Proposição 6.2.4 A aplicação $\otimes$ definida anteriormente é bilinear e ||$\otimes|| \leq 1$.

Prova. Sejam $\bar{T} \in \mathrm{E}_{Y}$ e $\overline{U_{1}}, \overline{U_{2}} \in \mathrm{E}_{\mathrm{ZY}}$.

$\otimes\left(\bar{T}, \overline{U_{1}}+\overline{U_{2}}\right)=\bar{T} \otimes\left(\overline{U_{1}}+\overline{U_{2}}\right)=\overline{T\left(U_{1}+U_{2}\right)}=\overline{T U_{1}+T U_{2}}=\overline{T U_{1}}+\overline{T U_{2}}=\bar{T} \otimes \overline{U_{1}}+\bar{T} \otimes \overline{U_{2}}$ $=\otimes\left(\bar{T}, \overline{U_{1}}\right)+\otimes\left(\bar{T}, \overline{U_{2}}\right)$.

Sejam $\bar{T} \in \mathrm{EY}_{\mathrm{Y}} \bar{U} \in \mathrm{E}_{\mathrm{ZY}}$ e $\lambda \in \mathrm{IK}$, assim:

$$
\otimes(\bar{T}, \lambda \bar{U})=\bar{T} \otimes \overline{\lambda U}=\overline{T(\lambda U)}=\overline{\lambda(T U)}=\lambda \overline{T U}=\lambda \bar{T} \otimes \bar{U}=\lambda \otimes(\bar{T}, \bar{U})
$$

Logo, $\otimes$ é linear na segunda variável.

De forma análoga provamos que $\otimes$ é linear na primeira variável.

Portanto, $\otimes$ é uma aplicação bilinear.

Vamos agora mostrar que ||$\otimes|| \leq 1$.

Lembrando que ||$\otimes||=\sup \{|||\otimes(\bar{T}, \bar{U})||| z:|||\bar{T}||| \mathrm{Y} \leq 1$ e ||$|\bar{U}||| z \leq 1\}$ temos: 
||$|\otimes(\bar{T}, \bar{U})||| \mathrm{z}=|||\overline{T U}||| \mathrm{z}=|| \mathrm{TU}|| \mathrm{z} \leq|| \mathrm{T}|| \mathrm{Y}|| \mathrm{U}|| \mathrm{z} \Rightarrow|| \otimes\left(\frac{\bar{T}}{\|T\|_{Y}}, \frac{\bar{U}}{\|U\|_{Z}}\right)|| \mid \mathrm{z}$

$\leq 1$. Assim segue que ||$\otimes|| \leq 1$.

Proposição 6.2.5 Sejam $Y$ e Zem $G_{X}$ e $Y^{\prime} \leq Y$. Então $E_{Z Y^{\prime}} \subset E_{Z Y}$.

Prova. Seja $\alpha_{Z} \in \mathrm{E}_{Z Y^{\prime}}$, daí temos:

a) Existe $\mathrm{T}$ representante de $\alpha_{Z} \operatorname{com} \mathrm{T}: \mathrm{Z} \rightarrow \mathrm{X}$ e $\operatorname{ImT} \subset \mathrm{Y}^{\prime}$;

b) Por hipótese, existe um isomorfismo bom, entre $Y^{\prime}$ e um subespaço $Y^{\prime \prime}$ de $Y, I_{Y^{\prime}}+S: Y^{\prime} \rightarrow Y^{\prime \prime}$.

Logo por (a) e (b) segue, $\left(\mathrm{I}_{\mathrm{Y}^{\prime}}+\mathrm{S}\right) \mathrm{T}: \mathrm{Z} \rightarrow \mathrm{X}$ está bem definida e portanto $\overline{\left(\mathrm{I}_{\mathrm{Y}^{\prime}}+\mathrm{S}\right) \mathrm{T}} \in \mathrm{E}_{\mathrm{Z}}$ e como $\operatorname{Im}\left(\mathrm{I}_{Y^{\prime}}+\mathrm{S}\right) \mathrm{T} \subset \mathrm{Y}$ segue que $\overline{\left(\mathrm{I}_{\mathrm{Y}^{\prime}}+\mathrm{S}\right) \mathrm{T}} \in \mathrm{E}_{\mathrm{ZY}}$.

Vamos agora mostrar que $\overline{\left(\mathrm{I}_{\mathrm{Y}^{\prime}}+\mathrm{S}\right) \mathrm{T}}=\bar{T}=\alpha_{\mathrm{Z}}$. De fato:

$\left(\mathrm{I}_{Y^{\prime}}+\mathrm{S}\right) \mathrm{T}-\mathrm{T}=\mathrm{I}_{Y^{\prime}} \mathrm{T}+\mathrm{ST}-\mathrm{T}=\mathrm{T}+\mathrm{ST}-\mathrm{T}=\mathrm{ST} \in \mathrm{S}(\mathrm{Z}, \mathrm{X})$. Logo, $\left(\mathrm{I}_{Y^{\prime}}+\mathrm{S}\right) \mathrm{T} \in \bar{T} \Rightarrow \overline{\left(\mathrm{I}_{\mathrm{Y}^{\prime}}+\mathrm{S}\right) \mathrm{T}}=\overline{\mathrm{T}}=\alpha_{Z}$.

Assim por $(\diamond)$ temos que $\alpha_{Z} \in E_{Z Y}$. Logo mostramos que $\alpha_{Z} \in E_{Z Y^{\prime}} \Rightarrow \alpha_{Z} \in E_{Z Y}$.

Portanto, $\mathrm{E}_{\mathrm{ZY} Y^{\prime}} \subset \mathrm{E}_{\mathrm{ZY}}$.

Proposição 6.2.6 Sejam $Y$ e Z em $G_{X}$, e seja $\alpha_{Y} \in E_{Y}$. Então existe $Y_{1} \leq Y$ tal que $P_{Y Y_{1}}\left(\alpha_{Y}\right) \in E_{Y_{1} Z}$. Prova.

(a) Se $\alpha_{Y}=\overline{0}$, isto é, $\alpha_{Y}=S(Y, X)$, basta tomarmos $Y_{1}=Y$ pois daí teremos:

$$
\mathrm{Y}_{1} \leq \mathrm{Y} \text { e } \mathrm{P}_{\mathrm{YY}_{1}}(\overline{0})=\overline{0} \in E_{Y_{1} Z} \text {. }
$$

(b) Suponhamos agora que $\alpha_{Y} \neq \overline{0}$.

Seja $\mathrm{T} \in \mathrm{L}(\mathrm{Y}, \mathrm{X})$ um representante de $\alpha_{Y}$. Como $\alpha_{Y} \neq \overline{0}$, segue que T não é estritamente singular, logo existe $Y_{2}$ subespaço fechado de $Y$, com dimensão infinita, tal que: 
$\mathrm{T}: \mathrm{Y}_{2} \rightarrow \mathrm{T}\left(\mathrm{Y}_{2}\right)$ é um isomorfismo.

(1)

Logo dados $\mathrm{T}\left(\mathrm{Y}_{2}\right)$ e $\mathrm{Z}$ em $\mathrm{G}$, pelo Lema 4.6 temos que existe um isomorfismo bom entre $W$ subespaço de $T\left(Y_{2}\right)$ e $Z^{\prime}$ subespaço de $Z$.

Consideremos então $\mathrm{Y}_{1}$ subespaço de $\mathrm{Y}_{2}$, tal que $\mathrm{T}\left(\mathrm{Y}_{1}\right)=\mathrm{W}$. ( possível por (1)).

Assim temos:

i) $Y_{1} \leq Y$ pois $Y_{1} \subset Y_{2} \subset Y$

$$
\mathrm{T}_{Y_{1}} \quad \mathrm{I}_{W}+\mathrm{S}
$$

ii) $\mathrm{Y}_{1} \longrightarrow \mathrm{W} \longrightarrow \mathrm{Z}^{\prime} \subset \mathrm{Z}$

$$
\left(\mathrm{I}_{\mathrm{W}}+\mathrm{S}\right) \mathrm{T}_{\left.\right|_{1}}
$$

Logo $\left(\mathrm{I}_{W}+S\right) \mathrm{T}_{\left.\right|_{1}}: \mathrm{Y}_{1} \rightarrow X$ está bem definida e $\operatorname{Im}\left[\left(\mathrm{I}_{W}+S\right) \mathrm{T}_{Y_{1}}\right] \subset \mathrm{Z}^{\prime} \subset \mathrm{Z}$.

Portanto: ${\overline{\left(I_{W}+S\right) T}}_{\left.\right|_{1}} \in \mathrm{E}_{\mathrm{Y}_{1} Z}$.

Agora, pela proposição anterior é fácil mostrar que $\overline{\left(\mathrm{I}_{\mathrm{W}}+\mathrm{S}\right) \mathrm{T}} /_{\mathrm{Y}_{1}}=\overline{\mathrm{T}}_{\mathrm{Y}_{1}}$.

Assim, $\overline{\mathrm{T}}_{\mathrm{Y}_{1}} \in E_{Y_{1} Z}$.

Mas, por outro lado como $\mathrm{Y}_{1} \subset \mathrm{Y}$ temos o seguinte: $P_{Y Y_{1}}\left(\alpha_{\mathrm{Y}}\right)=P_{Y Y_{1}}(\bar{T})=\overline{\mathrm{T}\left(\mathrm{I}_{\mathrm{Y}_{1}}+0\right)}=\overline{\mathrm{T}}_{\mathrm{Y}_{1}}$. Segue então por (2) que $P_{Y Y_{1}}\left(\alpha_{Y}\right) \in E_{Y_{1} Z}$.

Assim exibimos $Y_{1} \leq Y$ tal que $P_{Y_{1}}\left(\alpha_{Y}\right) \in E_{Y_{1} Z}$. 


\section{O ESPAÇO QUOCIENTE “E”}

\subsection{A construção do espaço quociente " $E$ "}

Nesta seção faremos o seguinte: dado $X$ um espaço de Banach H.I., para cada $Y \in G_{X}$, tomaremos um elemento $\alpha_{Y} \in E_{Y}$, teremos assim uma família no espaço $1_{\infty}\left(\left(E_{Y}\right)_{Y \in G_{X}}\right)$. Definiremos então o que é uma família "coerente" para em seguida construirmos o espaço E.

Definição 7.1.1 Seja X um espaço de Banach hereditariamente indecomponível. Definimos:

$$
1_{\infty}\left(\left(E_{Y}\right)_{Y \in G_{X}}\right)=\left\{\left(\alpha_{Y}\right)_{Y \in G_{X}} / \exists M \in R_{+}^{*}:||\left|\alpha_{Y}\right||| \gamma \leq M, \forall Y \in G_{X}\right\} .
$$

Observação 7.1.2 É fácil verificar que $\mathrm{l}_{\infty}\left(\left(\mathrm{E}_{\mathrm{Y}}\right)_{\mathrm{Y} \in \mathrm{G}_{\mathrm{X}}}\right)$ é um espaço vetorial normado onde ||$\left(\alpha_{Y}\right)_{Y}||=\sup _{Y \in G_{X}}||\left|\alpha_{Y}\right||| \gamma$.

Definição 7.1.3 Um elemento $\left(\alpha_{\mathrm{Y}}\right)_{\mathrm{Y} \in \mathrm{G}_{\mathrm{X}}} \in \mathrm{l}_{\infty}\left(\left(\mathrm{E}_{\mathrm{Y}}\right)_{\mathrm{Y} \in \mathrm{G}_{\mathrm{X}}}\right)$ é chamado coerente se existe $Y_{0} \in \mathrm{G}_{\mathrm{X}}$ tal que para todo $Y \leq Y_{0}$ temos $\alpha_{\mathrm{Y}}=\mathrm{P}_{\mathrm{Y}_{0} \mathrm{Y}}\left(\alpha_{\mathrm{Y}_{0}}\right)$.

Observação 7.1.4 Denotaremos por $\Omega$ o conjunto dos elementos coerentes de $1_{\infty}\left(\left(\mathrm{E}_{\mathrm{Y}}\right)_{\mathrm{Y} \in \mathrm{G}_{\mathrm{X}}}\right)$.

Proposição 7.1.5 $\Omega$ é um espaço linear.

Prova. Basta mostrarmos que $\Omega$ é um subespaço de $1_{\infty}\left(\left(E_{Y}\right)_{Y \in G_{X}}\right)$.

(i) $0 \in \Omega$, pois sendo $\mathrm{P}_{\mathrm{Y}_{0} \mathrm{Y}}$ linear, segue que $\forall \mathrm{Y}_{0} \in \mathrm{GX}_{\mathrm{X}}, \forall \mathrm{Y} \leq \mathrm{Y}_{0}$ temos $\mathrm{P}_{\mathrm{Y}_{0} \mathrm{Y}}(0)=0$. 
(ii) Sejam $\left(\alpha_{Y}\right)_{Y} \in \Omega$ e $\lambda$ um escalar.

$$
\left(\alpha_{Y}\right)_{Y} \in \Omega \Rightarrow \exists Y_{0} \in G_{X}: \forall Y \leq Y_{0}, \alpha_{Y}=P_{Y_{0} Y}\left(\alpha_{Y_{0}}\right)
$$

Afirmação : $\lambda\left(\alpha_{Y}\right)_{Y} \in \Omega$. De fato, como $\lambda\left(\alpha_{Y}\right)_{Y}=\left(\lambda \alpha_{Y}\right)_{Y}$, utilizando (1) e do fato de que $\mathrm{P}_{\mathrm{Y}_{0} \mathrm{Y}}$ é linear temos:

$$
\mathrm{P}_{\mathrm{Y}_{0} \mathrm{Y}}\left(\lambda \alpha_{\mathrm{Y}_{0}}\right)=\lambda \mathrm{P}_{\mathrm{Y}_{0} \mathrm{Y}}\left(\alpha_{\mathrm{Y}_{0}}\right)=\lambda \alpha_{\mathrm{Y}} \forall \mathrm{Y} \leq \mathrm{Y}_{0}
$$

Assim exibimos $\mathrm{Y}_{0} \in \mathrm{GX}_{\mathrm{X}}$ tal que $\forall \mathrm{Y} \leq \mathrm{Y}_{0}, \lambda \alpha_{\mathrm{Y}}=\mathrm{P}_{\mathrm{Y}_{0} \mathrm{Y}}\left(\lambda \alpha_{\mathrm{Y}_{0}}\right), \log \lambda\left(\alpha_{\mathrm{Y}}\right)_{\mathrm{Y}} \in \Omega$.

(iii) Vamos agora mostrar que dado $\left(\alpha_{Y}\right)_{Y} \in \Omega$ e $\left(\beta_{Y}\right)_{Y} \in \Omega$ temos $\left(\alpha_{Y}\right)_{Y}+\left(\beta_{Y}\right)_{Y} \in \Omega$.

$$
\begin{aligned}
& \left(\alpha_{Y}\right)_{Y} \in \Omega \Rightarrow \exists Y_{1} \in G_{X}: \forall Y \leq Y_{1}, \alpha_{Y}=P_{Y_{1} Y}\left(\alpha_{Y_{1}}\right) . \\
& \left(\beta_{Y}\right)_{Y} \in \Omega \Rightarrow \exists Y_{2} \in G_{X}: \forall Y \leq Y_{2}, \beta_{Y}=P_{Y_{2} Y}\left(\beta_{Y_{2}}\right) .
\end{aligned}
$$

Agora, dados $Y_{1}$ e $Y_{2} \in G_{X}$, pelo Lema 4.6 temos que $\exists Y_{0} \in G_{X}$ tal que $Y_{0} \leq Y_{1}$ e $Y_{0} \leq Y_{2}$.

Assim segue de (2) e (3) que $\alpha_{\mathrm{Y}_{0}}=\mathrm{P}_{\mathrm{Y}_{1} \mathrm{Y}_{0}}\left(\alpha_{\mathrm{Y}_{1}}\right)$ e $\beta_{\mathrm{Y}_{0}}=\mathrm{P}_{\mathrm{Y}_{2} \mathrm{Y}_{0}}\left(\beta_{\mathrm{Y}_{2}}\right)$.

Logo, dado $\forall Y \leq Y_{0}$, decorre de (4), da Proposição 6.1.6, de (2) e (3) que:

$$
\begin{aligned}
& P_{Y_{0} Y}\left(\alpha_{Y_{0}}+\beta_{Y_{0}}\right)=P_{Y_{0} Y}\left(\alpha_{Y_{0}}\right)+P_{Y_{0} Y}\left(\beta_{Y_{0}}\right)=P_{Y_{0} Y}\left(P_{Y_{1} Y_{0}}\left(\alpha_{Y_{1}}\right)\right)+P_{Y_{0} Y}\left(P_{Y_{2} Y_{0}}\left(\beta_{Y_{2}}\right)\right)= \\
& P_{Y_{0} Y} P_{Y_{1} Y_{0}}\left(\alpha_{Y_{1}}\right)+P_{Y_{0} Y} P_{Y_{2} Y_{0}}\left(\beta_{Y_{2}}\right)=P_{Y_{1} Y}\left(\alpha_{Y_{1}}\right)+P_{Y_{2} Y}\left(\beta_{Y_{2}}\right)=\alpha_{Y}+\beta_{Y} .
\end{aligned}
$$

Assim exibimos $Y_{0} \in G_{X}$ tal que $\forall Y \leq Y_{0}$ temos $P_{Y_{0} Y}\left(\alpha_{Y_{0}}+\beta_{Y_{0}}\right)=\alpha_{Y}+\beta_{Y}$.

Logo, $\left(\alpha_{Y}\right)_{Y}+\left(\beta_{Y}\right)_{Y} \in \Omega$.

Por (i), (ii) e (iii) segue que $\Omega$ é um subespaço de $1_{\infty}\left(\left(E_{Y}\right)_{Y \in G_{X}}\right)$. 
Vamos agora definir uma semi-norma no espaço $\Omega$.

Definição 7.1.6 Dado $\left(\alpha_{\mathrm{Y}}\right)_{\mathrm{Y} \in \mathrm{G} X} \in \Omega$ segue do Lema 6.1.7 que $\left\|\mid \alpha_{\mathrm{Y}}\right\|_{\mathrm{Y}}=\|\| \alpha_{\mathrm{Y}_{0}}\|\|_{\mathrm{Y}_{0}} \forall Y \leq Y_{0}$, pois $\alpha_{\mathrm{Y}}=\mathrm{P}_{\mathrm{Y}_{0} \mathrm{Y}}\left(\alpha_{\mathrm{Y}_{0}}\right)$ e $\mathrm{P}_{\mathrm{Y}_{0} \mathrm{Y}}$ é uma isometria. Assim podemos definir a seguinte aplicação em $\Omega$ :

$$
\begin{aligned}
\psi: \Omega & \rightarrow R_{+} \\
\left(\alpha_{Y}\right)_{Y} & \rightarrow \psi\left(\left(\alpha_{Y}\right)_{Y}\right)=\left\|\alpha_{Y_{0}}\right\|_{Y_{0}} .
\end{aligned}
$$

Observação 7.1.7 Vamos mostrar que a aplicação $\psi$ está bem definida.

$$
\left(\alpha_{Y}\right)_{Y} \in \Omega \Rightarrow \exists Y_{0} \in G_{X}: \forall Y \leq Y_{0}, \alpha_{Y}=P_{Y_{0} Y}\left(\alpha_{Y_{0}}\right)
$$

Suponhamos então que exista $\mathrm{Y}_{1} \in \mathrm{G}_{\mathrm{X}}$ tal que $\forall \mathrm{Y} \leq \mathrm{Y}_{1}$ temos $\alpha_{\mathrm{Y}}=\mathrm{P}_{\mathrm{Y}_{1} \mathrm{Y}}\left(\alpha_{\mathrm{Y}_{1}}\right)$.

Vamos mostrar que $\left\|\alpha_{Y_{0}}\right\|_{Y_{0}}=\left\|\alpha_{Y_{1}}\right\|_{Y_{1}}$. De fato, dados $Y_{0}$ e $Y_{1} \in G \times$ pelo Lema 4.6 temos que $\exists W \in G \times$ tal que $W \leq Y_{0}$ e $W \leq Y_{1}$.

Assim segue de (1), (2) e do Lema 6.1.7 que:

$$
\begin{gathered}
\mathrm{P}_{\mathrm{Y}_{0} \mathrm{~W}}\left(\alpha_{\mathrm{Y}_{0}}\right)=\alpha_{\mathrm{W}} \Rightarrow\left\|\alpha_{\mathrm{W}}\right\|\left\|_{\mathrm{W}}=\right\| \mathrm{P}_{\mathrm{Y}_{0} \mathrm{~W}}\left(\alpha_{\mathrm{Y}_{0}}\right)\|\|_{\mathrm{W}}=\left\|\alpha_{\mathrm{Y}_{0}}\right\| \|_{\mathrm{Y}_{0}} . \\
\mathrm{e} \\
\mathrm{P}_{\mathrm{Y}_{1} \mathrm{~W}}\left(\alpha_{\mathrm{Y}_{1}}\right)=\alpha_{\mathrm{W}} \Rightarrow\left\|\alpha_{\mathrm{W}}\right\|\left\|_{\mathrm{W}}=\right\| \mathrm{P}_{\mathrm{Y}_{1} \mathrm{~W}}\left(\alpha_{\mathrm{Y}_{1}}\right)\|\|_{\mathrm{W}}=\left\|\alpha_{\mathrm{Y}_{1}}\right\| \|_{\mathrm{Y}_{1}} .
\end{gathered}
$$

Logo, $\left\|\left|\alpha_{Y_{0}}\right|\right\|_{Y_{0}}=\left|\left\|\alpha_{Y_{1}}\right\|\right|_{Y_{1}}$.

Proposição 7.1.8 A aplicação $\psi$ definida anteriormente é uma semi norma em $\Omega$. .

Prova. (i) Sejam $\left(\alpha_{Y}\right)_{Y} \in \Omega$ e $\lambda$ um escalar. Assim temos:

$$
\lambda\left(\alpha_{\mathrm{Y}}\right)_{\mathrm{Y}} \in \Omega \text { e } \Psi\left(\lambda\left(\alpha_{\mathrm{Y}}\right)_{\mathrm{Y}}\right)=\left|\left\|\lambda \alpha_{\mathrm{Y}_{0}}\right\|_{\mathrm{Y}_{0}}=\right| \lambda|.|\left|\alpha_{\mathrm{Y}_{0}} \|_{\mathrm{Y}_{0}}=\right| \lambda \mid . \Psi\left(\left(\alpha_{\mathrm{Y}}\right)_{\mathrm{Y}}\right) .
$$

Logo, $\Psi\left(\lambda\left(\alpha_{Y}\right)_{Y}\right)=|\lambda| . \Psi\left(\left(\alpha_{Y}\right)_{Y}\right)$. 
(ii) Sejam $\left(\alpha_{Y}\right)_{Y}$ e $\left(\beta_{Y}\right)_{Y} \in \Omega$. Pela Proposição 7.1.5 segue que $\exists Y_{0} \in G_{X}$ tal que $\forall Y \leq Y_{0}$ temos $\alpha_{\mathrm{Y}}=\mathrm{P}_{\mathrm{Y}_{0} \mathrm{Y}}\left(\alpha_{\mathrm{Y}_{0}}\right), \beta_{\mathrm{Y}}=\mathrm{P}_{\mathrm{Y}_{0} \mathrm{Y}}\left(\beta_{\mathrm{Y}_{0}}\right)$ e portanto $\mathrm{P}_{\mathrm{Y}_{0} \mathrm{Y}}\left(\alpha_{\mathrm{Y}_{0}}+\beta_{\mathrm{Y}_{0}}\right)=\alpha_{\mathrm{Y}}+\beta_{\mathrm{Y}}$. Assim temos que $\left(\alpha_{Y}\right)_{Y}+\left(\beta_{Y}\right)_{Y} \in \Omega$ e vale:

$\Psi\left(\left(\alpha_{Y}\right)_{Y}+\left(\beta_{Y}\right)_{Y}\right)=\|\| \alpha_{Y_{0}}+\beta_{Y_{0}}\left\|_{Y_{0}} \leq\right\| \mid \alpha_{Y_{0}}\left\|_{Y_{0}}+\right\| \beta_{Y_{0}} \|_{Y_{0}}=\Psi\left(\left(\alpha_{Y}\right)_{Y}\right)+\Psi\left(\left(\beta_{Y}\right)_{Y}\right)$

Logo, $\Psi\left(\left(\alpha_{Y}\right)_{Y}+\left(\beta_{Y}\right)_{Y}\right) \leq \Psi\left(\left(\alpha_{Y}\right)_{Y}\right)+\Psi\left(\left(\beta_{Y}\right)_{Y}\right)$.

Assim, por (i) e (ii) segue que $\Psi$ é uma semi-norma em $\Omega$.

Observação 7.1.9 Denotaremos por $\Gamma=\left\{\left(\alpha_{Y}\right)_{Y \in G_{X}} \in \Omega: \psi\left(\left(\alpha_{Y}\right)_{Y}\right)=0\right\}$.

Proposição 7.1.10 Гé um subespaço fechado de $\Omega$.

Prova. Primeiramente vamos mostrar que $\Gamma$ é subespaço de $\Omega$.

- $0 \in \Gamma$ pois $0=\left(\alpha_{Y}\right)_{Y}$ onde $\alpha_{Y}=S(Y, X) \forall Y \in G_{X}$ e portanto segue que:

$$
\Psi(0)=||\left|\alpha_{Y}\right|||_{Y}=|| S||_{Y}=0 \forall S \in \alpha_{Y} .
$$

- $\operatorname{Dados}\left(\alpha_{Y}\right)_{Y} \in \Gamma$ e $\lambda$ um escalar, segue da Proposição 7.1 .8 (i) que:

$$
\Psi\left(\lambda\left(\alpha_{Y}\right)_{Y}\right)=|\lambda| . \Psi\left(\left(\alpha_{Y}\right)_{Y}\right)=0, \text { pois } \Psi\left(\left(\alpha_{Y}\right)_{Y}\right)=0 . \log 0 \lambda\left(\alpha_{Y}\right)_{Y} \in \Gamma
$$

- Sejam $\left(\alpha_{Y}\right)_{Y}$ e $\left(\beta_{Y}\right)_{Y} \in \Gamma$, então $\Psi\left(\left(\alpha_{Y}\right)_{Y}\right)=0$ e $\Psi\left(\left(\beta_{Y}\right)_{Y}\right)=0$. Assim, segue da Proposição 7.1 .8 (ii) que:

$\Psi\left(\left(\alpha_{Y}\right)_{Y}+\left(\beta_{Y}\right)_{Y}\right) \leq \Psi\left(\left(\alpha_{Y}\right)_{Y}\right)+\Psi\left(\left(\beta_{Y}\right)_{Y}\right)=0 \Rightarrow \Psi\left(\left(\alpha_{Y}\right)_{Y}+\left(\beta_{Y}\right)_{Y}\right)=0 \Rightarrow\left(\alpha_{Y}\right)_{Y}+\left(\beta_{Y}\right)_{Y} \in \Gamma$.

Vamos agora mostrar que $\Gamma$ é fechado. 
Para tanto é suficiente provar que a aplicação $\Psi$ é contínua em $\Omega$, pois assim $\Gamma$ será a imagem inversa de um fechado $\left(\Gamma=\Psi^{-1}(\{0\})\right)$ por uma aplicação contínua, sendo portanto fechado.

Afirmação: $\Psi$ é contínua em $\Omega$. De fato, seja $\left(\beta_{Y}\right)_{Y} \in \Omega$. Vamos mostrar que dado $\varepsilon>0$, $\exists \delta>0$ tal que:

$$
||\left(\alpha_{Y}\right)_{Y}-\left(\beta_{Y}\right)_{Y}||<\delta \Rightarrow\left|\Psi\left(\left(\alpha_{Y}\right)_{Y}\right)-\Psi\left(\left(\beta_{Y}\right)_{Y}\right)\right|<\varepsilon .
$$

De fato, basta tomarmos $\delta=\varepsilon$, pois sendo $\Psi$ uma semi-norma, segue da desigualdade triangular e do fato de que ||$\left(\alpha_{Y}\right)_{Y}||=\sup _{Y \in G_{X}}||\left|\alpha_{Y}\right||| \gamma$ :

$$
\left|\Psi\left(\left(\alpha_{Y}\right)_{Y}\right)-\Psi\left(\left(\beta_{Y}\right)_{Y}\right)\right| \leq \Psi\left(\left(\alpha_{Y}\right)_{Y}-\left(\beta_{Y}\right)_{Y}\right)=||\left|\alpha_{Y_{0}}-\beta_{Y_{0}}\right| \|_{Y_{0}} \leq||\left(\alpha_{Y}-\beta_{Y}\right)_{Y}||<\delta
$$

Agora, como $\left(\beta_{Y}\right)_{Y} \in \Omega$ é arbitrário, segue que $\Psi$ é contínua em $\Omega$.

Definição 7.1.11 Sendo $\Gamma=\left\{\left(\alpha_{\mathrm{Y}}\right)_{\mathrm{Y}_{\mathrm{G}} \mathrm{X}} \in \Omega: \psi\left(\left(\alpha_{\mathrm{Y}}\right)_{\mathrm{Y}}\right)=0\right\}$ subespaço fechado de $\Omega$, definimos então o seguinte espaço quociente : $\mathrm{E}=\Omega / \Gamma$.

\section{Observações 7.1.12}

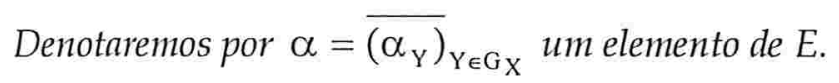

Proposição 7.1.13 Considere a seguinte aplicação ||$.||: E \rightarrow \mathbf{R}_{+}$dada por ||$\alpha||=\psi\left(\left(\alpha_{Y}\right)_{Y}\right)$, $\forall\left(\alpha_{Y}\right)_{Y} \in \alpha .|| .||$ está bem definida e é uma norma em $E$.

Prova. Segue diretamente das Proposições 7.1.8 e 1.3.5.

Observação 7.1.14 Decorre das Proposições 7.1.13 e 6.1 .3 que ||$\alpha||=\left\|\alpha_{Y_{0}}\right\|\left\|_{Y_{0}}=\right\| T \|_{Y_{0}}$ $\forall T \in \alpha_{Y_{0}}$. 
Observação 7.1.14 Decorre das Proposições 7.1.13 e 6.1 .3 que ||$\alpha||=\left\|\mid \alpha_{\mathrm{Y}_{0}}\right\|_{\mathrm{Y}_{0}}=\|\mathrm{T}\|_{\mathrm{Y}_{0}}$ $\forall T \in \alpha_{Y_{0}}$.

Observação 7.1.15 Denotaremos por $\left(\alpha_{Y}\right)_{Y_{Z}}$ a família definida por $\left\{\begin{array}{ccc}\alpha_{Y} \in E_{Y} & \text { se } & Y \leq Z \\ 0 & \text { caso } & \text { contrário }\end{array}\right.$.

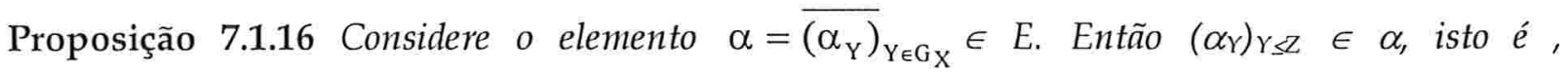
${\overline{\left(\alpha_{Y}\right)_{Y \in G X}}}={\overline{\left(\alpha_{Y}\right)_{Y} \leq Z}}$

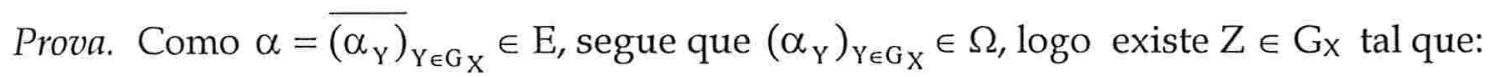

$$
\alpha_{Y}=P_{Z Y}\left(\alpha_{Z}\right) \quad \forall Y \leq Z
$$

Consideremos então o seguinte elemento de $\Omega$ : $\left(\alpha_{Y}\right)_{Y \leq Z}$.

Vamos mostrar que $\left(\alpha_{Y}\right)_{Y \leq Z} \in \alpha$.

Considere a família $\left(\beta_{\mathrm{Y}}\right)_{\mathrm{Y} \in \mathrm{G}_{\mathrm{X}}}$, onde $\beta_{\mathrm{Y}}=\left\{\begin{array}{ccc}\alpha_{\mathrm{Y}} \in \mathrm{E}_{\mathrm{Y}} & \text { se } & \mathrm{Y} \leq \mathrm{Z} \\ 0 & \text { caso } & \text { contrário }\end{array}\right.$.

É fácil verificar que $\left(\beta_{Y}\right)_{Y \in G_{X}}=(\alpha Y)_{Y \leq Z} \in \Omega$, e portanto $\left(\alpha_{Y}\right)_{Y \in G_{X}}-\left(\beta_{Y}\right)_{Y \in G_{X}} \in \Omega$, e mais, $Z \in G_{X}$ é tal que $\alpha_{Y}-\beta_{Y}=P_{Z Y}\left(\alpha_{Z}-\beta_{Z}\right), \forall Y \leq Z$.

Assim, lembrando que $\beta_{Y}=\alpha_{Y} \quad \forall Y \leq Z$, temos:

$$
\begin{aligned}
& \Psi\left(\left(\alpha_{Y}\right)_{Y \in G_{X}}-\left(\beta_{Y}\right)_{Y \in G_{X}}\right)=||\left|\alpha_{Z}-\beta_{Z}\right||| z=||\left|\alpha_{Z}-\alpha_{Z}\right||| z=|||0||| z=0 . \\
& \text { Logo, }\left(\alpha_{Y}\right)_{Y \in G_{X}}-\left(\beta_{Y}\right)_{Y \in G X} \in \Gamma \Rightarrow\left(\beta_{Y}\right)_{Y \in G_{X}} \in \alpha \Rightarrow\left(\alpha_{Y}\right)_{Y \leq Z} \in \alpha \Rightarrow \overline{\left(\alpha_{Y}\right)_{Y \in G_{X}}}=\overline{\left(\alpha_{Y}\right)_{Y \leq Z}} .
\end{aligned}
$$




\subsection{Um produto para os elementos de $\mathrm{E}$}

Nesta seção, utilizando o produto definido para os elementos de EY (ver definição 6.2.1) vamos definir um produto para os elementos de E. Veremos que esse produto satisfaz algumas propriedades importantes para nossa teoria.

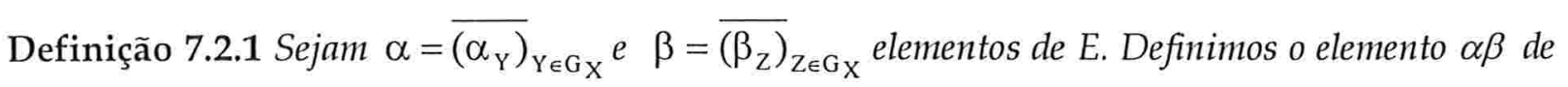

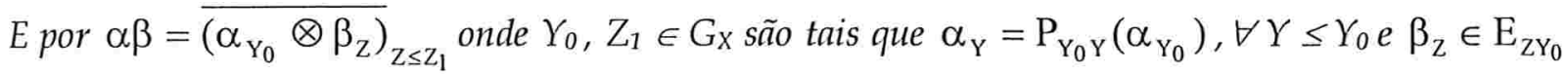
$\forall \mathrm{Z} \leq \mathrm{Z}_{1}$

Observação 7.2.2 Mostraremos agora que o elemento $\alpha \beta \in$ E está bem definido, e que esse produto não depende da escolha dos representantes de $\alpha$ e $\beta$.

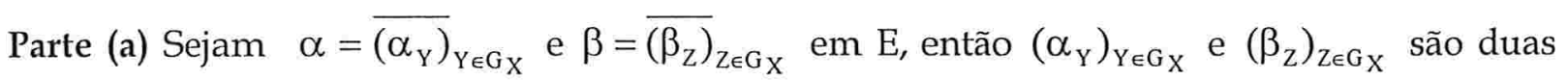
famílias coerentes, logo existem $\mathrm{Y}_{0}$ e $\mathrm{Z}_{0} \in \mathrm{GX}_{\mathrm{X}}$ tais que:

$$
\forall Y \leq Y_{0} \text { e } \forall Z \leq Z_{0} \text { temos } \alpha_{Y}=P_{Y_{0} Y}\left(\alpha_{Y_{0}}\right) \text { e } \beta_{Z}=P_{Z_{0} Z}\left(\beta_{Z_{0}}\right)
$$

Agora, dado $Y_{0}$ e $Z_{0} \in G_{X}$ e $\beta_{z_{0}} \in E_{Z_{0}}$, segue da Proposição 6.2.6:

$$
\exists \mathrm{Z}_{1} \leq \mathrm{Z}_{0} \text { tal que } \beta_{\mathrm{Z}_{1}}=\mathrm{P}_{\mathrm{Z}_{0} \mathrm{Z}_{1}}\left(\beta_{\mathrm{Z}_{0}}\right) \in \mathrm{E}_{\mathrm{Z}_{1} \mathrm{Y}_{0}}
$$

Logo, existe $T$ um representante de $\beta_{Z_{1}}$ tal que $T: Z_{1} \rightarrow X$ com $\operatorname{ImT} \subset Y_{0}$. (3)

Assim, $\forall Z \leq Z_{1}$, segue de (2), da Proposição 6.1 .6 e de (1) que:

$$
\mathrm{P}_{\mathrm{z}_{1} \mathrm{z}}\left(\beta_{\mathrm{z}_{1}}\right)=\mathrm{P}_{\mathrm{z}_{1} \mathrm{z}}\left(\mathrm{P}_{\mathrm{z}_{0} \mathrm{z}_{1}}\left(\beta_{\mathrm{z}_{0}}\right)\right)=\mathrm{P}_{\mathrm{z}_{0} \mathrm{z}}\left(\beta_{\mathrm{z}_{0}}\right)=\beta_{\mathrm{z}}
$$

Agora, segue de (3) que: 


$$
\beta_{\mathrm{Z}}=\mathrm{P}_{\mathrm{Z}_{1} \mathrm{Z}}\left(\beta_{\mathrm{Z}_{1}}\right)=\mathrm{P}_{\mathrm{Z}_{1} \mathrm{Z}}(\overline{\mathrm{T}})=\overline{\mathrm{T}\left(\mathrm{I}_{\mathrm{Z}}+\mathrm{S}\right)} \text { onde } \mathrm{T}\left(\mathrm{I}_{\mathrm{Z}}+\mathrm{S}\right): \mathrm{Z} \rightarrow \mathrm{Y}_{0}
$$

Assim dado $\alpha_{Y_{0}} \in E_{Y_{0}}$, para todo $Z \leq Z_{1}$, temos $\beta_{Z} \in E_{Z_{Y_{0}}}$, logo o produto $\alpha_{Y_{0}} \otimes \beta_{Z}$ está bem definido.

Parte (b) Vamos agora mostrar que $\left(\alpha_{\mathrm{Y}_{0}} \otimes \beta_{\mathrm{Z}}\right)_{\mathrm{Z} \leq \mathrm{Z}_{1}} \in \Omega$. De fato, seja U um representante de $\alpha_{\mathrm{Y}_{0}}$ e T um representante de $\beta_{\mathrm{Z}_{1}}$ tal que $\operatorname{ImT} \subset \mathrm{Y}_{0}$, então temos $U: \mathrm{Y}_{0} \rightarrow X$ e T : $Z_{1} \rightarrow \mathrm{Y}_{0}$.

Assim para todo $\mathrm{Z} \leq \mathrm{Z}_{1}$ segue que:

$$
\mathrm{P}_{\mathrm{Z}_{1} \mathrm{Z}}\left(\alpha_{\mathrm{Y}_{0}} \otimes \beta_{\mathrm{Z}_{1}}\right)=\mathrm{P}_{\mathrm{z}_{1} \mathrm{Z}}(\overline{\mathrm{U}} \otimes \overline{\mathrm{T}})=\mathrm{P}_{\mathrm{z}_{1} \mathrm{Z}}(\overline{\mathrm{UT}})=\overline{(\mathrm{UT})\left(\mathrm{I}_{\mathrm{Z}}+\mathrm{S}\right)}=\overline{\mathrm{U}\left(\mathrm{T}\left(\mathrm{I}_{\mathrm{Z}}+\mathrm{S}\right)\right)}
$$

Agora, denotando por $\mathrm{F}=\mathrm{T}\left(\mathrm{I}_{\mathrm{Z}}+\mathrm{S}\right)$ e lembrando que $\mathrm{I}_{\mathrm{Z}}+\mathrm{S}: \mathrm{Z} \rightarrow \mathrm{Z}^{\prime} \subset \mathrm{Z}_{1}$ temos:

$\mathrm{F}: \mathrm{Z} \rightarrow \mathrm{Y}_{0}$, e portando $\overline{\mathrm{F}} \in \mathrm{E}_{\mathrm{ZY}_{0}}$, e por (4) temos que $\overline{\mathrm{F}}=\beta_{\mathrm{Z}}$, assim segue de (5) que:

$$
\mathrm{P}_{\mathrm{Z}_{1} \mathrm{Z}}\left(\alpha_{\mathrm{Y}_{0}} \otimes \beta_{\mathrm{Z}_{1}}\right)=\overline{\mathrm{UF}}=\overline{\mathrm{U}} \otimes \overline{\mathrm{F}}=\alpha_{\mathrm{Y}_{0}} \otimes \beta_{\mathrm{Z}} \forall \mathrm{Z} \leq \mathrm{Z}_{1}
$$

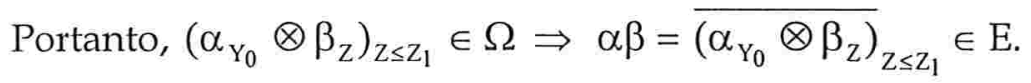

Parte (c) Mostraremos agora que para todo $Y \leq Y_{0}, \exists Z_{1}$ tal que $\forall Z \leq Z_{1}$, temos $\alpha_{Y} \otimes \beta_{Z}=\alpha_{Y_{0}} \otimes \beta_{Z}$.

Seja $Y \leq Y_{0}$. Pela parte (a) sabemos que existe $Z \in G_{X}$ tal que $\beta_{z} \in E_{Z Y}$. Daí, segue da Proposição 6.2 .5 que $\mathrm{E}_{\mathrm{ZY}} \subset \mathrm{E}_{\mathrm{ZY}_{0}}$, logo $\beta_{\mathrm{Z}} \in \mathrm{E}_{\mathrm{ZY}_{0}}$ e portanto os elementos $\alpha_{\mathrm{Y}} \otimes \beta_{\mathrm{Z}}$ e $\alpha_{Y_{0}} \otimes \beta_{Z}$ estão bem definidos.

Sendo T um representante de $\alpha_{\mathrm{Y}_{0}}$ e lembrando que $\alpha_{\mathrm{Y}}=\mathrm{P}_{\mathrm{Y}_{0} \mathrm{Y}}\left(\alpha_{\mathrm{Y}_{0}}\right), \forall \mathrm{Y} \leq \mathrm{Y}_{0}$, segue:

$$
\mathrm{P}_{\mathrm{Y}_{0} \mathrm{Y}}(\overline{\mathrm{T}})=\overline{\mathrm{T}\left(\mathrm{I}_{\mathrm{Y}}+\mathrm{S}\right)} \text { onde } \mathrm{I}_{\mathrm{Y}}+\mathrm{S}: \mathrm{Y} \rightarrow \mathrm{Y}^{\prime} \subset \mathrm{Y}_{0} \text {. }
$$


Assim $\mathrm{T}\left(\mathrm{I}_{Y}+S\right)$ é um representante de $\alpha_{Y}$, e considerando $U$, um representante de $\beta_{Z}$ tal que $\operatorname{ImU} \subset \mathrm{Y}$ temos:

$\alpha_{Y} \otimes \beta_{Z}=\overline{T\left(I_{Y}+S\right)} \otimes \bar{U}=\overline{\left(T\left(I_{Y}+S\right)\right) U}=\overline{T\left(\left(I_{Y}+S\right) U\right)}=\bar{T} \otimes \overline{\left(I_{Y}+S\right) U}$,

onde $\left(\mathrm{I}_{Y}+\mathrm{S}\right) \mathrm{U}: \mathrm{Z} \rightarrow \mathrm{Y}^{\prime} \subset \mathrm{Y}_{0}$.

Agora, como $\left(I_{Y}+S\right) U-U=I_{Y} U+S U-U=U+S U-U=S U$ segue da Proposição 3.1.6 que $\left(\mathrm{I}_{Y}+\mathrm{S}\right) \mathrm{U}-\mathrm{U}$ é estritamente singular, $\log 0\left(\mathrm{I}_{Y}+\mathrm{S}\right) \mathrm{U} \in \overline{\mathrm{U}}=\beta_{Z}$.

Assim, segue de $(\diamond)$ que $\forall Y \leq Y_{0}, \exists Z_{1}$ tal que $\forall Z \leq Z_{1} \quad \alpha_{Y} \otimes \beta_{Z}=\alpha_{Y_{0}} \otimes \beta_{Z}$.

Parte (d)Vamos mostrar agora que se existem $Y_{1}$ e $Z_{2} \in G_{X}$ tais que $\alpha \beta=\overline{\left(\alpha_{Y_{1}} \otimes \beta_{Z}\right)_{Z \leq Z_{2}}}$ então

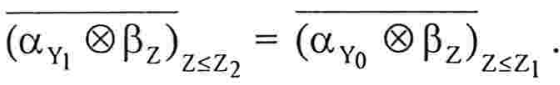

Dados $Y_{0}$ e $Y_{1} \in G_{x}$, pelo Lema 4.6 sabemos que existe $Y_{2} \in G_{x}$ tal que $Y_{2} \leq Y_{0}$ e $Y_{2} \leq Y_{1}$. Assim pelo que foi provado na parte (c) temos:

$$
\begin{aligned}
& \exists Z_{1}^{\prime} \text {, tal que } \forall Z \leq Z_{1}^{\prime},\left(\alpha_{Y_{2}} \otimes \beta_{Z}\right)=\left(\alpha_{Y_{0}} \otimes \beta_{Z}\right) \\
& \exists Z_{2}^{\prime} \text {, tal que } \forall Z \leq Z,\left(\alpha_{Y_{2}} \otimes \beta_{Z}\right)=\left(\alpha_{Y_{1}} \otimes \beta_{Z}\right)
\end{aligned}
$$

Seja $Z_{3}$ tal que $Z_{3} \leq Z_{1}^{\prime}$ e $Z_{3} \leq Z_{2}^{\prime}$, então $\forall Z \leq Z_{3}$ temos $\left(\alpha_{Y_{1}} \otimes \beta_{Z}\right)=\left(\alpha_{Y_{0}} \otimes \beta_{Z}\right)$.

Portanto, segue da Proposição 7.1.16 que $\overline{\left(\alpha_{Y_{1}} \otimes \beta_{Z}\right)_{Z \leq Z_{2}}}={\overline{\left(\alpha_{Y_{0}} \otimes \beta_{Z}\right)}}_{Z \leq Z_{1}}$.

Parte (e) Finalmente, vamos mostrar que $\alpha \beta$ não depende da escolha dos representantes de $\alpha$ e $\beta$.

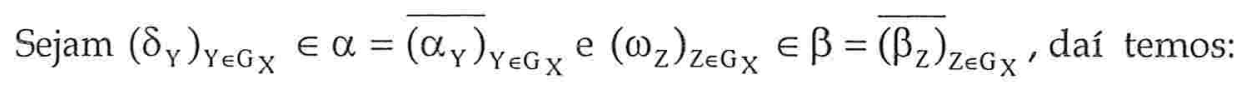


$\left(\delta_{Y}\right)_{Y \in G_{X}}-\left(\alpha_{Y}\right)_{Y \in G_{X}} \in \Gamma \Rightarrow \Psi\left(\left(\delta_{Y}\right)_{Y_{E G X}}-\left(\alpha_{Y}\right)_{Y_{Y \in G X}}\right)=0 \Rightarrow\left\|\delta_{Y_{0}}-\alpha_{Y_{0}}\right\|_{Y_{0}}=0 \Rightarrow$ $\delta_{\mathrm{Y}_{0}}=\alpha_{\mathrm{Y}_{0}}$.

Assim, $\exists Y_{0}$ tal que $\omega_{Y}=\beta_{Y}, \forall Y \leq Y_{0}$.

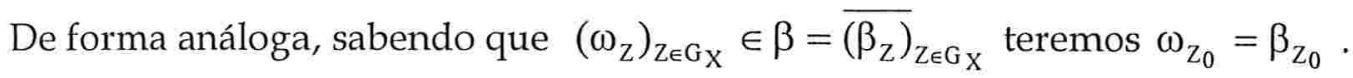
Assim, $\exists Z_{0}$ tal que $\omega_{\mathrm{Z}}=\beta_{\mathrm{Z}}, \forall \mathrm{Z} \leq \mathrm{Z}_{0}$.

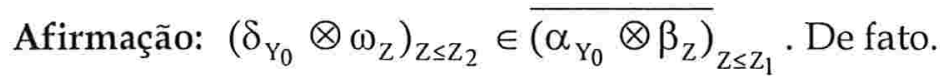

$\left(\delta_{\mathrm{Y}_{0}} \otimes \omega_{\mathrm{Z}}\right)_{\mathrm{Z} \leq \mathrm{Z}_{2}}-\left(\alpha_{\mathrm{Y}_{0}} \otimes \beta_{\mathrm{Z}}\right)_{\mathrm{Z} \leq \mathrm{Z}_{1}} \in \Omega$ e portanto:

$\Psi\left(\left(\delta_{Y_{0}} \otimes \omega_{Z}\right)_{Z \leq Z_{2}}-\left(\alpha_{Y_{0}} \otimes \beta_{Z}\right)_{Z \leq Z_{1}}\right)=\mid\left\|\delta_{Y_{0}} \otimes \omega_{Z}-\alpha_{Y_{0}} \otimes \beta_{Z}\right\|_{Z}, \forall Z \leq Z_{0}$.

Logo de (6) e (7) segue que $\Psi\left(\left(\delta_{\mathrm{Y}_{0}} \otimes \omega_{\mathrm{Z}}\right)_{\mathrm{Z} \leq \mathrm{Z}_{2}}-\left(\alpha_{\mathrm{Y}_{0}} \otimes \beta_{\mathrm{Z}}\right)_{\mathrm{Z} \leq \mathrm{Z}_{1}}\right)=0$.

Assim, $\left(\delta_{Y_{0}} \otimes \omega_{Z}\right)_{Z \leq Z_{2}}-\left(\alpha_{Y_{0}} \otimes \beta_{Z}\right)_{Z \leq Z_{1}} \in \Gamma \Rightarrow\left(\delta_{Y_{0}} \otimes \omega_{Z}\right)_{Z \leq Z_{2}} \in \overline{\left(\alpha_{Y_{0}} \otimes \beta_{Z}\right)_{Z} \leq Z_{1}}$.

Proposição 7.2.3 Sejam $\alpha$ e $\beta$ em E. Então ||$\alpha \beta|| \leq|| \alpha||$.|| $\beta||$.

Prova. Dados $\alpha$ e $\beta \in$ E, segue das Proposições 7.1.13 e 6.2.4:

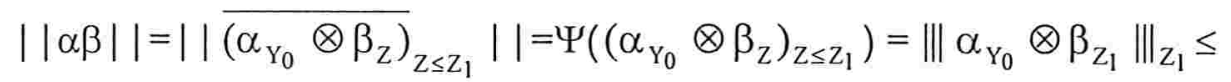

$\|\otimes\| \cdot\left\|\alpha_{Y_{0}}\right\|_{Y_{0}} \cdot\left\|\beta_{Z_{1}}\right\|_{Z_{1}} \leq\left\|\alpha_{Y_{0}}\right\|_{Y_{0}} \cdot\left\|\beta_{Z_{1}}\right\|_{Z_{1}}=\|\alpha\| \cdot\|\beta\|$.

Portanto, ||$\alpha \beta|| \leq|| \alpha|| .|| \beta||$. 
Proposição 7.2.4 O espaço E com a multiplicação dada na definição 7.2.1 e com a norma definida na Proposição 7.1.13 possui estrutura de álgebra normada com unidade.

Prova. Como E já possui estrutura de um espaço vetorial, basta então verificarmos as seguintes propriedades:

P1. $(\alpha \beta) \omega=\alpha(\beta \omega), \forall \alpha, \beta, \omega \in \mathrm{E}$.

P2. $\alpha(\beta+\omega)=\alpha \beta+\alpha \omega$ e $(\beta+\omega) \alpha=\beta \alpha+\omega \alpha, \forall \alpha, \beta, \omega \in$ E.

P3. $\lambda(\alpha \beta)=(\lambda \alpha) \beta=\alpha(\lambda \beta), \forall \alpha, \beta \in E, \forall \lambda \in I K$.

P4. $\exists 1 \in \mathrm{E}:|| 1||=1$ e $\alpha 1=1 \alpha=\alpha, \forall \alpha \in \mathrm{E}$.

P5. ||$\alpha \beta|| \leq|| \alpha|| .|| \beta||, \forall \alpha, \beta \in \mathrm{E}$.

\section{Verificação das propriedades:}

P1. Sejam $\alpha, \beta$ e $\omega \in$ E.

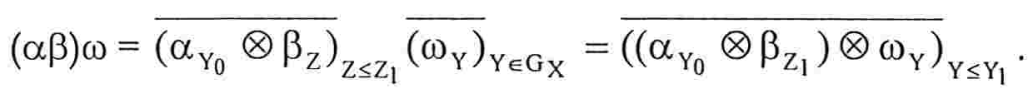

Considerando agora, $\mathrm{T}_{1}: \mathrm{Y}_{0} \rightarrow \mathrm{X}, \mathrm{T}_{2}: \mathrm{Z}_{1} \rightarrow \mathrm{X}$ e $\mathrm{T}_{3}: \mathrm{Y} \rightarrow \mathrm{X}$ representantes de $\alpha_{Y_{0}}, \beta_{Z_{1}}$ e $\omega_{\mathrm{Y}}$, respectivamente, tais que $\operatorname{ImT}_{2} \subset \mathrm{Y}_{0}$ e $\operatorname{ImT}_{3} \subset \mathrm{Z}_{1}$ temos:

$\left(\alpha_{Y_{0}} \otimes \beta_{Z_{1}}\right) \otimes \omega_{Y}=\left(\overline{T_{1}} \otimes \overline{T_{2}}\right) \otimes \overline{T_{3}}=\left(\overline{T_{1} T_{2}}\right) \otimes \overline{T_{3}}=\overline{\left(T_{1} T_{2}\right) T_{3}}=\overline{T_{1}\left(T_{2} T_{3}\right)}=\overline{T_{1}} \otimes\left(\overline{T_{2}} \otimes \overline{T_{3}}\right)=\alpha_{Y_{0}} \otimes\left(\beta_{Z_{1}} \otimes \omega_{Y}\right)$

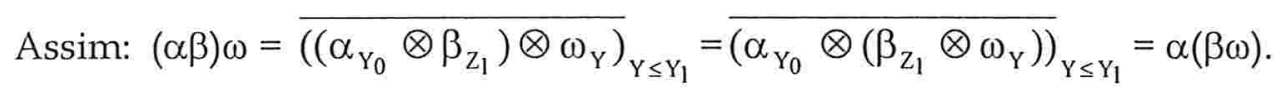

P2. Sejam $\alpha, \beta$ e $\omega \in$ E. Utilizando o fato de que $\otimes$ é linear na segunda variável, temos:

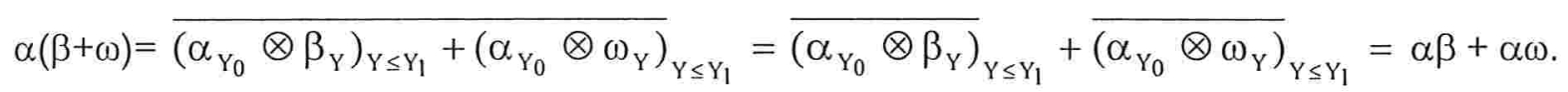

De forma análoga podemos mostrar que $(\beta+\omega) \alpha=\beta \alpha+\omega \alpha$. 
P3. Sejam $\alpha, \beta \in$ E e $\lambda$ um escalar. Utilizando o fato de $\otimes$ é bilinear, temos:

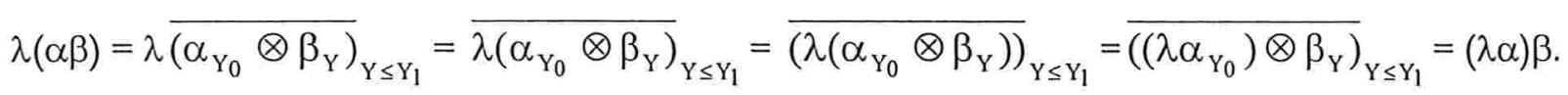

De forma análoga podemos mostrar que $\lambda(\alpha \beta)=\alpha(\lambda \beta)$.

P4. Seja $\left.1=\overline{(\bar{I}}_{Z}\right)_{Z \in G_{X}}$. Vamos mostrar que $1 \in$ E e que $1 \alpha=\alpha \mathbf{1}=\alpha, \forall \alpha \in \mathrm{E}$.

Para mostrarmos que $1 \in \mathrm{E}$, basta provarmos que $\left(\overline{\mathrm{I}}_{z}\right)_{\mathrm{Z \in G} \mathrm{X}} \in \Omega$, o que de fato ocorre pois dado $Z \in G_{X}$ temos que $Z \leq X$ e assim segue que:

$$
\operatorname{Pxz}\left(\overline{\mathrm{I}}_{\mathrm{X}}\right)=\overline{\mathrm{I}_{\mathrm{X}} \mathrm{I}_{\mathrm{Z}}}=\overline{\mathrm{I}}_{\mathrm{Z}} \forall \mathrm{Z} \leq \mathrm{X}
$$

Logo $\left(\overline{\mathrm{I}}_{\mathrm{z}}\right)_{\mathrm{Z} \in \mathrm{G}_{X}}$ é uma família coerente $\Rightarrow\left(\overline{\mathrm{I}}_{\mathrm{z}}\right)_{\mathrm{Z} \in \mathrm{G}_{X}} \in \Omega \Rightarrow \mathbf{1}=\overline{(\overline{\mathrm{I}} z}_{\mathrm{Z} \in \mathrm{G}_{X}} \in \mathrm{E}$.

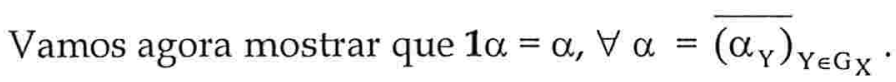

$$
\left.1 \alpha=\overline{(\overline{\mathrm{I}}}_{\mathrm{X}} \otimes \alpha_{\mathrm{Y}}\right)_{\mathrm{Y} \leq \mathrm{Y}_{1}} .
$$

Agora, sendo $\mathrm{T}: \mathrm{Y} \rightarrow \mathrm{X}$ um representante de $\alpha_{Y}$, temos:

$$
\overline{\mathrm{I}}_{\mathrm{X}} \otimes \alpha_{\mathrm{Y}}=\overline{\mathrm{I}}_{\mathrm{X}} \otimes \overline{\mathrm{T}}=\overline{\mathrm{I}_{\mathrm{X}} \mathrm{T}}=\overline{\mathrm{T}}=\alpha_{\mathrm{Y}} .
$$

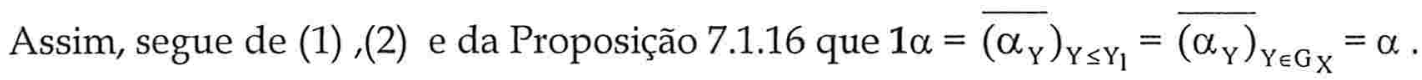

Vamos agora, mostrar que $\alpha 1=\alpha, \forall \alpha \in \mathrm{E}$.

$$
\alpha \mathbf{1}={\overline{\left(\alpha_{Y_{0}} \otimes \bar{I}_{Z}\right)_{Z} \leq Z_{1}}}
$$


Considerando $\mathrm{T}: \mathrm{Y}_{0} \rightarrow \mathrm{X}$ um representante de $\alpha_{Y_{0}}$ e $\mathrm{F}: \mathrm{Z} \rightarrow \mathrm{X}$ um representante de $\overline{\mathrm{I}}_{\mathrm{Z}}$ tal que $\operatorname{ImF} \subset \mathrm{Y}_{0}$ temos $\mathrm{F} \in \bar{I}_{Z} \Rightarrow \mathrm{F}-\mathrm{I}_{\mathrm{Z}}=\mathrm{S} \in \mathrm{S}(\mathrm{Z}, \mathrm{X}) \Rightarrow \mathrm{F}=\mathrm{I}_{\mathrm{Z}}+\mathrm{S} \Rightarrow \mathrm{Z} \leq \mathrm{Y}_{0}$.

Assim, $\alpha_{\mathrm{Y}_{0}} \otimes \overline{\mathrm{I}}_{\mathrm{Z}}=\overline{\mathrm{T}} \otimes \overline{\mathrm{F}}=\overline{\mathrm{TF}}=\overline{\mathrm{T}\left(\mathrm{I}_{\mathrm{Z}}+\mathrm{S}\right)}=\mathrm{P}_{\mathrm{Y}_{0} \mathrm{Z}}(\overline{\mathrm{T}})=\alpha_{\mathrm{Z}} \quad \forall \mathrm{Z} \leq \mathrm{Y}_{0}$

Logo, segue de (3), (4) e também da Proposição 7.1.16, que:

$$
\left.\left.\alpha 1=\overline{\left(\alpha_{Y_{0}} \otimes \bar{I}_{Z}\right)_{Z \leq Z_{1}}}=\overline{\left(\alpha_{Z}\right.}\right)_{Z \leq Z_{1}}=\overline{\left(\alpha_{Y}\right.}\right)_{Y \in G_{X}}=\alpha
$$

Finalmente, falta mostrarmos que || $\mathbf{1}||=1$, o que de fato ocorre, pois, segue das proposições 7.1.13 e 6.1 .3 que:

$$
\left.|| 1||=|| \overline{(\bar{I}}_{z}\right)_{Z \in G_{X}}||=\Psi\left(\left(\overline{\mathrm{I}}_{z}\right)_{Z_{E \in G_{X}}}\right)=||\left|\overline{\mathrm{I}}_{\mathrm{X}}\right||| x=|| \mathrm{I}_{\mathrm{X}}|| x=1 .
$$

P5. Ver demonstração da Proposição 7.2.3. 


\subsection{Uma isometria entre os espaços E e C}

O objetivo desta seção é provar através de alguns conceitos novos e do Teorema de Gelfand-Mazur (Teorema 2.2.3) que o espaço E construído anteriormente é isométricamente isomorfo ao espaço $\mathrm{C}$ dos números complexos.

Definição 7.3.1 Seja X um espaço de Banach H.I. Para cada $Y \in G_{X}$ definimos a seguinte aplicação:

$$
\begin{aligned}
\text { e }: L(Y, X) & \rightarrow E \\
T & \rightarrow \mathrm{e}(T)=\overline{\left(\mathrm{P}_{\mathrm{YZ}}(\overline{\mathrm{T}})\right)_{\mathrm{Z} \leq Y} .}
\end{aligned}
$$

Observação 7.3.2 Vamos mostrar que a aplicação "è" está bem definida.

Dado $\mathrm{T} \in \mathrm{L}(\mathrm{Y}, \mathrm{X})$, denotando a classe $\bar{T}$ por $\alpha_{Y}$ e por $\alpha_{Z}$ a classe $\mathrm{P}_{Y Z}(\bar{T})$, temos:

$$
\left(\alpha_{Z}\right)_{Z \leq Y} \in \Omega \text { pois, } \forall Z \leq Y, \quad \alpha_{Z}=P_{Y Z}\left(\alpha_{Y}\right), \log 0 \overline{\left(P_{Y Z}(\bar{T})\right)_{Z \leq Y}} \in E .
$$

Proposição 7.3.3 A aplicação "e" definida anteriormente é linear.

Prova. Decorre diretamente do fato de que a aplicação Pyz é linear, ver Lema 6.1.7.

Proposição 7.3.4 Todo elemento de E é da forma e(T) para algum $T$ definido sobre algum $Y \in G_{X}$.

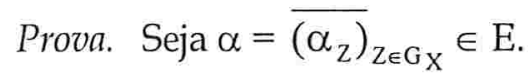

Existe $Y \in G_{X}$, tal que $\forall Z \leq Y$ temos $\alpha_{Z}=P_{Y Z}\left(\alpha_{Y}\right)$.

Consideremos então o seguinte elemento de E: $(\alpha Z)_{Z \leq Y}$.

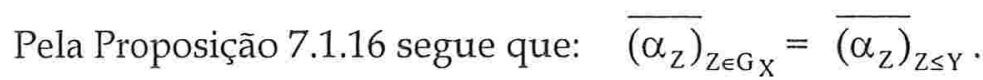




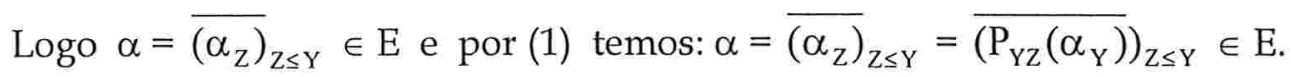

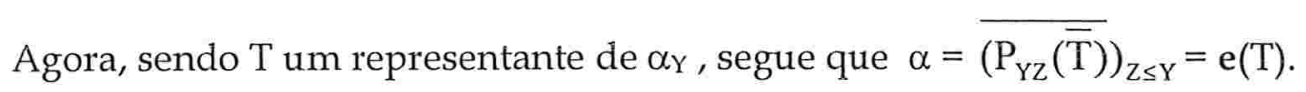

Como $\alpha \in \mathrm{E}$ é arbitrário, segue que todo elemento de $\mathrm{E}$ é da forma $\mathrm{e}(\mathrm{T})$.

Observação 7.3.5 Sendo $\left.1=\overline{(\bar{I}}_{Z}\right)_{\mathrm{Z} \in \mathrm{G}_{\mathrm{X}}}$ e lembrando que $\operatorname{PXZ}\left(\bar{I}_{X}\right)=\bar{I}_{Z}, \forall \mathrm{Z} \leq \mathrm{X}$, segue da

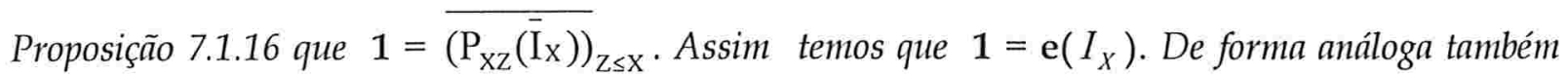
podemos mostrar que fixado $Y \in G \times$, temos $1=\mathrm{e}\left(I_{Y}\right)$.

Observação 7.3.6 Decorre da Observação 7.1.14 que || e(T) || = || T || .

Lema 7.3.7 Seja $Y \in G_{X}$ e $T \in L(Y, X)$ tal que $\alpha=e(T)$. Se $F=T_{\mathrm{I}_{\mathrm{w}}}$ onde $W$ é subespaço de $Y$, então $\alpha=\mathrm{e}(F)$.

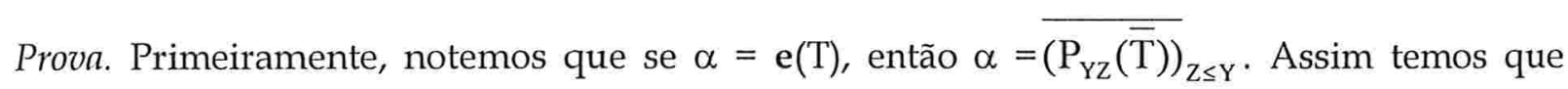
$\alpha_{\mathrm{Z}}=\mathrm{P}_{\mathrm{YZ}}(\overline{\mathrm{T}}) \forall \mathrm{Z} \leq \mathrm{Y}$. Em particular sendo $\mathrm{W}$ subespaço de $\mathrm{Y}$, temos:

$$
\mathrm{W} \leq \mathrm{Y} \text { e } \alpha_{\mathrm{W}}=\mathrm{P}_{\mathrm{YW}}(\overline{\mathrm{T}})=\overline{\mathrm{TI}_{\mathrm{W}}}=\overline{\mathrm{T}_{\mathrm{w}}}=\overline{\mathrm{F}}
$$

Agora, dado $Z \leq W \leq Y$, segue de (1) que:

$$
\operatorname{PWZ}(\bar{F})=\operatorname{PWZ}(\operatorname{PYw}(\bar{T}))=\operatorname{PYZ}(\bar{T})
$$

Assim, segue de (2) e da Proposição 7.1.16 que:

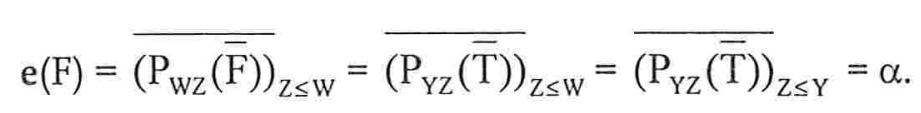


Lema 7.3.8 Seja $\left(Y_{n}\right)_{n \in N}$ uma seqüência em $G_{X}$ tal que $Y_{n+1} \subset Y_{n}, \forall n \in N$. Suponha ainda que $Y_{0}$ tenha base de Schauder $\left(x_{n}\right)_{n}$. Então, $Y_{0}$ possui uma seqüêencia básica $\left(y_{n}\right)_{n}$ tal que $y_{n} \in Y_{n}, \forall n \in \mathbf{N}$.

Prova. Seja $\mathrm{y}_{0} \in \mathrm{Y}_{0}$ tal que || $\mathrm{y}_{0} \|=1, \operatorname{como}\left(\mathrm{x}_{\mathrm{n}}\right)_{\mathrm{n}}$ é base de Schauder de $\mathrm{Y}_{0}$ com constante básica $\mathrm{k} \geq 1$, segue que $\mathrm{y}_{0}=\sum_{\mathrm{i}=0}^{\infty} \mathrm{a}_{\mathrm{i}} \mathrm{x}_{\mathrm{i}}$. Logo dado $\varepsilon=\frac{1}{8 k}$, temos:

$$
\exists n_{0} \in N \text {, tal que } n \geq n_{0} \Rightarrow|| \sum_{i=0}^{\infty} a_{i} x_{i}-\sum_{i=0}^{n} a_{i} x_{i}||<\frac{1}{8 k}
$$

Agora, denotando por $\mathrm{b}_{0}=\sum_{\mathrm{i}=0}^{\mathrm{n}} \mathrm{a}_{\mathrm{i}} \mathrm{x}_{\mathrm{i}}$ segue que || $\mathrm{y}_{0}-\mathrm{b}_{0}||<\frac{1}{8 k}$.

Por hipótese temos que $Y_{1} \subset Y_{0}$ e $\left(x_{n}\right)_{n}$ é base de Schauder de $Y_{0}$, logo segue da Proposição 1.2.16 que dado $\mathrm{n}_{0} \in \mathrm{N}$, existe $\mathrm{y}_{1} \in \mathrm{Y}_{1}$, com || $\mathrm{y}_{1}||=1$ tal que $\mathrm{y}_{1}=\sum_{\mathrm{i}=\mathrm{n}_{0}+1}^{\infty} \mathrm{a}_{\mathrm{i}} \mathrm{x}_{\mathrm{i}}$. Assim, dado $\varepsilon=\frac{1}{16 k}, \exists \mathrm{n}_{1} \in \mathrm{N}$, tal que $\mathrm{n} \geq \mathrm{n}_{1} \Rightarrow|| \mathrm{y}_{1}-\mathrm{b}_{1}||<\frac{1}{16 k}$, onde $\mathrm{b}_{1}=\sum_{\mathrm{i}=\mathrm{n}_{0}+1}^{\mathrm{n}_{1}} \mathrm{a}_{\mathrm{i}} \mathrm{x}_{\mathrm{i}}$.

Procedendo de forma análoga, iremos obter duas seqüências $\left(y_{n}\right)_{n}$ e $\left(b_{n}\right)_{n}$ tais que:

(i) $\left(b_{n}\right)_{n}$ é uma base de blocos de $\left(x_{n}\right)_{n}$. Logo pela Proposição 1.2.15, temos que $\left(b_{n}\right)_{n}$ é uma seqüência básica com constante básica k', menor ou igual a k.

(ii) $\left(y_{n}\right)_{n}$ é tal que $y_{n} \in Y_{n}$ e ||$y_{n}-b_{n}||<\frac{1}{2^{n+3} \cdot k}, \forall n \in N$.

Agora, por (i) e pela Proposição 1.2.18 segue que $\left\{\frac{b_{n}}{\left\|b_{n}\right\|}\right\}_{n \in N}$ é seqüência básica normalizada de $\mathrm{Y}_{0}$ com a mesma constante básica $\mathrm{k}^{\prime}$. E mais:

$\left\|y_{n}-\frac{b_{b}}{\left\|b_{n}\right\|}\right\|=\left\|y_{n}-b_{n}+b_{n}-\frac{b}{\left\|b_{n}\right\|}\right\| \leq\left\|y_{n}-b_{n}\right\|+\left\|b_{n}-\frac{b_{n}}{\left\|b_{n}\right\|}\right\|<\left\|y_{n}-b_{n}\right\|+\left\|b_{n}\right\|-1 \mid$ 
Mas por construção temos:

$$
\left\|b_{n}\right\|=\left\|b_{n}-y_{n}+y_{n}\right\| \leq\left\|b_{n}-y_{n}\right\|+\left\|y_{n}\right\| \leq 1+\frac{1}{2^{n+3} \cdot k}
$$

E de forma análoga podemos mostrar que $\left\|b_{n}\right\| \geq 1-\frac{1}{2^{n+3} k}$.

Assim , $\left\|b_{n}\right\|-1 \mid \leq \frac{1}{2^{n+3} \cdot k}$ e portanto $\left\|y_{n}-\frac{b_{n}}{\left\|b_{n}\right\|}\right\|<\frac{2}{2^{n+3} k}$.

Logo, $\sum_{n=0}^{\infty}\left\|y_{n}-\frac{b_{n}}{\left\|b_{n}\right\|}\right\|<\sum_{n=0}^{\infty} \frac{1}{2^{n+2} k}=\frac{1}{2 k} \leq \frac{1}{2 k^{\prime}}$. Daí segue, da Proposição 1.2.13 que $\left(y_{n}\right)_{n}$ é uma seqüência básica de $Y_{0}$ e por (ii) temos ainda que $y_{n} \in Y_{n}, \forall n \in N$.

Definição 7.3.9 Seja $\alpha \in E$, dado $Y \in G X$, nós dizemos que um elemento $T \in L(Y, X)$ é um 2 - representante minimal de $\alpha$ sobre $Y$ se $\alpha=\mathrm{e}(T)$ e ||$T|| \leq 2|| \alpha||$.

Proposição 7.3.10 Dado $\alpha \in E, \exists Y_{0} \in G_{X} e T_{0} \in L\left(Y_{0}, X\right)$ tal que $\alpha=\mathrm{e}\left(T_{0}\right)$ e ||$T_{0}|\leq 2||\alpha| \mid$, ou seja a possui um 2 - representante minimal.

Prova. Seja $\alpha \in \mathrm{E}, \alpha \neq 0$, pela Proposição 7.3.4 sabemos que $\alpha=\mathrm{e}(\mathrm{T})$ para algum $\mathrm{Y} \in \mathrm{GX}$ e para algum $\mathrm{T} \in \mathrm{L}(\mathrm{Y}, \mathrm{X})$ daí, pela Observação 7.1.14 segue que,

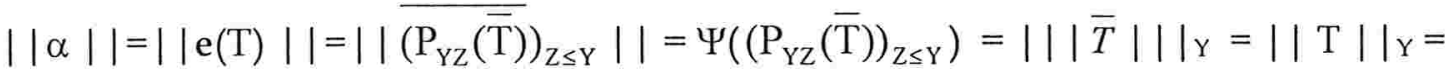

$$
\begin{aligned}
& \inf _{Y^{\prime} \subset Y}|| T_{\mid Y^{\prime}}|| \text {. }
\end{aligned}
$$

Assim, decorre da definição de ínfimo, que dado $\varepsilon=|| \alpha||>0, \exists Y_{0} \subset Y$ tal que :

$$
\left\|\mathrm{T}_{\mathrm{IY}_{0}}\right\|<|| \alpha||+\varepsilon \text {, ou seja, } \exists \mathrm{Y}_{0} \subset \mathrm{Y} \text { tal que }\left\|\mathrm{T}_{\mathrm{IY}_{0}}\right\|<2|| \alpha||
$$

Agora, segue do Lema 7.3.7, que e $\left(\mathrm{T}_{\mathrm{Y}_{0}}\right)=\alpha$. Assim, denotando $\mathrm{T}_{0}=\mathrm{T}_{\mathrm{I}_{0}}$ temos:

$\exists Y_{0} \in G_{X}$ e $T_{0} \in L\left(Y_{0}, X\right)$ tal que $\alpha=e\left(T_{0}\right)$ e ||$T_{0}||<2|| \alpha||$.

A igualdade se dá quando $\alpha=0$. 
Lema 7.3.11 Seja $\left(\alpha_{n}\right)_{n}$ uma seqüência, tal que $\alpha_{n} \in E, \forall n \in \mathrm{N}$. Então dado $T_{n-1} u m$ 2 - representante minimal de $\alpha_{n-1}$ sobre $Y_{n-1}$, existe $T_{n}$ um 2 - representante minimal de $\alpha_{n}$ sobre $Y_{n}$ subespaço de $Y_{n-1}$

Prova. (i) Dado $\alpha_{0} \in \mathrm{E}$, pela Proposição anterior sabemos que existe $\mathrm{Y}_{0} \in \mathrm{G}_{\mathrm{X}}$ e $\mathrm{T}_{0} \in \mathrm{L}\left(\mathrm{Y}_{0}, \mathrm{X}\right)$ tal que $\alpha_{0}=e\left(T_{0}\right)$ e ||$T_{0}|| \leq 2|| \alpha_{0}||$.

(ii) Considere agora $\alpha_{1} \in \mathrm{E}$. Pela Proposição 7.3.4 sabemos que existe $\mathrm{T} \in \mathrm{L}(\mathrm{Y}, \mathrm{X})$ para algum $Y \in G x$ tal que, $\alpha_{1}=e(T)$.

Agora, dado $Y_{0}$ e $Y \in G_{X}$, pelo Lema 4.6, sabemos que existe $W \in G_{X}, W$ subespaço de $Y_{0}$ tal que $\mathrm{W} \leq \mathrm{Y}_{0}$ e $\mathrm{W} \leq \mathrm{Y}$.

Como $W \leq Y$, então existe $u m I_{W}+S_{1}$ - isomorfismo entre $W$ e $Y^{\prime}$ subespaço de $Y$.

Consideremos então o seguinte operador de $W$ em $X: F=T\left(I_{W}+S_{1}\right)$.

(iii) Vamos agora mostrar que $\alpha_{1}=\mathrm{e}(\mathrm{F})$.

Primeiramente observemos que dado $Z \leq W$, como $W \leq Y$ temos $Z \leq Y$ e assim utilizando (2) temos que:

$$
\mathrm{P}_{\mathrm{WZ}}(\bar{F})=\overline{F\left(I_{Z}+S_{2}\right)}=\overline{T\left(I_{W}+S_{1}\right)\left(I_{Z}+S_{2}\right)}=\overline{T\left(I_{Z}^{\prime}+S\right)}=\operatorname{PYZ}(\bar{T}),
$$

onde $I_{Z}^{\prime}+S=\left(I_{W}+S_{1}\right)\left(I_{Z}+S_{2}\right)$, pela Proposição 4.4 , é um isomorfismo bom entre Z e um subespaço de $Y$.

Assim segue de (1) e (3) que $\left.\quad e(F)=\overline{\left(\mathrm{P}_{\mathrm{WZ}}(\overline{\mathrm{F}})\right)_{\mathrm{Z} \leq \mathrm{W}}}=\overline{\left(\mathrm{P}_{\mathrm{YZ}}(\overline{\mathrm{T}})\right.}\right)_{\mathrm{Z} \leq \mathrm{Y}}=\mathrm{e}(\mathrm{T})=\alpha_{1}$.

Logo segue que, ||$\alpha_{1}||=|| \mathrm{e}(\mathrm{F})||=|||\bar{F}||| w=|| F|| w=\inf _{W^{\prime} \subset W}|| F_{\mid w} . \mid$. 
Assim, pela definição de ínfimo, segue que dado $\varepsilon=|| \alpha_{1}||>0$ temos que:

$$
\exists W^{\prime \prime} \subset W \text {, tal que }|| F_{\mid W^{\prime \prime}}|| \leq 2|| \alpha_{1}|| \text {, }
$$

e como $\alpha_{1}=e(F)$, segue pelo Lema 7.3.7, que $\alpha_{1}=e\left(F_{\mid W^{\prime \prime}}\right)$.

Logo, denotando por $\mathrm{T}_{1}=\mathrm{F}_{\mid \mathrm{W}}$, e $\mathrm{Y}_{1}=\mathrm{W}^{\prime \prime}$ conseguimos:

$$
\mathrm{Y}_{1} \subset \mathrm{Y}_{0} \text { e } \mathrm{T}_{1} \in \mathrm{L}\left(\mathrm{Y}_{1}, \mathrm{X}\right) \operatorname{com} \alpha_{1}=\mathrm{e}\left(\mathrm{T}_{1}\right) \text { e }|| \mathrm{T}_{1}|| \leq 2|| \alpha_{1}||
$$

Procedendo de forma análoga obteremos uma seqüência $\left(T_{n}\right)_{n}$, tal que, $\forall n \in N$, temos: $T_{n} \in L\left(Y_{n}, X\right), Y_{n+1} \subset Y_{n}, \alpha_{n}=e\left(T_{n}\right)$ e ||$T_{n}|| \leq 2|| \alpha_{n}||$.

Lema 7.3.12 O espaço Eé completo.

Prova. Pela Proposição 1.2.19, é suficiente mostrarmos que toda série normalmente convergente em $\mathrm{E}$, converge em $\mathrm{E}$.

Seja $\left(\alpha_{n}\right)_{n}$ uma série normalmente convergente em $E$, isto é:

$$
\alpha_{\mathrm{n}} \in \mathrm{E}, \forall \mathrm{n} \in \mathrm{N} \text { e } \sum_{n=0}^{\infty}\left\|\alpha_{n}\right\|<\infty .
$$

Vamos mostrar que $\sum_{n=0}^{\infty} \alpha_{n}$ converge em E.

Dado $\left(\alpha_{n}\right)_{n} \in E$, pelo Lema 7.3.11, existe $\left(T_{n}\right)_{n}$ uma seqüência de 2-representantes minimais, tais que:

$$
\begin{aligned}
& \mathrm{T}_{\mathrm{n}} \in \mathrm{L}\left(\mathrm{Y}_{\mathrm{n}}, X\right) \text { onde } \mathrm{Y}_{\mathrm{n}+1} \subset \mathrm{Y}_{\mathrm{n}}, \forall \mathrm{n} \in \mathrm{N} . \\
& \alpha_{\mathrm{n}}=\mathrm{e}\left(\mathrm{T}_{\mathrm{n}}\right) \text { e }|| \mathrm{T}_{\mathrm{n}}|| \leq 2|| \alpha_{\mathrm{n}}||, \forall \mathrm{n} \in \mathrm{N} .
\end{aligned}
$$

Suponhamos agora que $Y_{0}$ tenha base de Schauder. Daí pelo Lema 7.3.8 segue:

$$
\exists\left(y_{n}\right)_{n} \text { seqüência básica de } Y_{0} \text {, tal que } y_{n} \in Y_{n}, \forall n \in \mathbb{N} \text {. }
$$


Caso $Y_{0}$ não tenha base de Schauder, pelo Teorema de Mazur ( Teorema 1.2.10) segue que $Y_{0}$ possui uma seqüência básica $\left(x_{n}\right)_{n}$. Considerando então o espaço gerado $Y_{0}^{\prime}=\left[\left(x_{n}\right)_{n}\right]$, temos:

$$
Y_{0}^{\prime} \subset \mathrm{Y}_{0} \text { e } \mathrm{T}_{\left.0\right|_{\mathrm{Y}_{0}}{ }^{\prime}} \text { tal que } \alpha_{0}=\mathrm{e}\left(\mathrm{T}_{\left.0\right|_{\mathrm{Y}_{0}}{ }^{\prime}}\right) \text { e }|| \mathrm{T}_{\left.0\right|_{\mathrm{Y}_{0}}}|| \leq|| \mathrm{T}_{0}|| \leq 2|| \alpha_{0}|| \text {. }
$$

E assim, partindo de $Y_{0}^{\prime}$, pelo Lema 7.3.11 conseguiremos uma seqüência $\left(Y_{n}^{\prime}\right)_{\mathbf{n}}$ satisfazendo (i) e (ii) e tal que $Y_{0}^{\prime}$ possui base de Schauder.

Consideremos agora os seguintes espaços gerados: $Y=\left[\left(y_{n}\right)_{n}\right], Y_{>n}=\left[\left(y_{k}\right)_{k>n}\right]$ e $Y_{\leq n}=\left[\left(y_{k}\right)_{k \leq n}\right]$. Agora, dado $\mathrm{y} \in \mathrm{Y}$, temos que $\mathrm{y}=\sum_{k=0}^{\infty} a_{k} y_{k}$, e assim podemos definir a seguinte seqüência de aplicações:

$$
\mathrm{P}_{>\mathrm{n}}: \mathrm{Y} \rightarrow \mathrm{Y}_{>\mathrm{n}} \text { onde } \mathrm{P}_{>\mathrm{n}}(\mathrm{y})=\sum_{k=n+1}^{\infty} a_{k} y_{k}
$$

Observando que $\mathrm{P}_{>\mathrm{n}}=\mathrm{I}_{\mathrm{Y}}-\mathrm{P}_{\mathrm{n}}$, onde $\mathrm{P}_{\mathrm{n}}: \mathrm{Y} \rightarrow \mathrm{Y}$ são as projeções contínuas definidas por $\mathrm{P}_{\mathrm{n}}(\mathrm{y})=\sum_{k=0}^{n} a_{k} y_{k}$ e que $\sup _{n \in N}|| \mathrm{P}_{\mathrm{n}}||=\mathrm{k}$, onde $\mathrm{k} \in \mathrm{R}$ e $\mathrm{k} \geq 1$ (ver Proposição 1.2.6 ), podemos facilmente verificar que:

$\forall \mathrm{n} \in \mathrm{N}$, a aplicação $\mathrm{P}_{>\mathrm{n}}$ é linear, contínua e || $\mathrm{P}_{>\mathrm{n}}|| \leq 1+\mathrm{k} \leq 2 \mathrm{k}$.

Vamos agora definir a seguinte seqüência de aplicações:

$$
\left(F_{n}\right)_{n \in N} \text { onde } \forall n \in N, F_{n}: Y \rightarrow X \text { e } F_{n}(y)=T_{n}\left(P_{>n}(y)\right) .
$$

Por (2), (4) e (5) é fácil verificar que $\forall n \in N, F_{n}$ está bem definida, e mais:

(i) $\mathrm{F}_{\mathrm{n}}$ é linear, pois é a composta de aplicações lineares .

(ii) $\mathrm{F}_{\mathrm{n}}$ é contínua, pois é a composta de aplicações contínuas.

Assim, podemos concluir que: $F_{n} \in L(Y, X)$. 
Agora, utilizando (2), (3) e (6) temos:

||$F_{n}(y)||=|| T_{n}\left(P_{>n}(y)\right)|| \leq|| T_{n}|| .|| P_{>n}(y)|| \leq 2|| \alpha_{n}|| .|| P_{>n}|| .|| y|| \leq$ 2||$\alpha_{\mathrm{n}}|| .2 \mathrm{k}|| \mathrm{y}||$.

Assim, ||$F_{n}(y)|| \leq 4 k .|| \alpha_{n}|| .|| y|| \Rightarrow|| F_{n}|| \leq 4 k|| \alpha_{n}||, \forall n \in N$.

Logo, $\sum_{n=0}^{\infty}\left\|F_{n}\right\|<4 \mathrm{k} \sum_{n=0}^{\infty}\left\|\alpha_{n}\right\|$. Mas por (1) temos $\sum_{n=0}^{\infty}\left\|\alpha_{n}\right\|<\infty$, assim:

$\sum_{n=0}^{\infty}\left\|F_{n}\right\|<\infty$ implica $\left(F_{n}\right)_{n}$ é normalmente convergente em $L(Y, X)$, e como $L(Y, X)$ é um espaço de Banach, pela Proposição 1.2.19 segue que $\sum_{n=0}^{\infty} F_{n}$ converge em $\mathrm{L}(\mathrm{Y}, \mathrm{X})$.

Por tanto, existe $\mathrm{F} \in \mathrm{L}(\mathrm{Y}, \mathrm{X})$ tal que $\sum_{n=0}^{\infty} F_{n}=\mathrm{F}$.

Agora, lembrando que $\alpha_{n}=e\left(T_{n}\right)$ e observando que $F_{n}=T_{n \mid Y>n}$, segue do Lema 7.3.7, que $\alpha_{\mathrm{n}}=\mathrm{e}\left(\mathrm{F}_{\mathrm{n}}\right)$, e denotando por $\alpha=\mathrm{e}(\mathrm{F}) \in \mathrm{E}$, para todo $\mathrm{n} \in \mathrm{N}$, segue da Proposição 7.3.3, Observação 7.3.6 e de (8) :

$$
\begin{aligned}
& || \alpha-\sum_{k=0}^{n} \alpha_{k}||=|| \mathrm{e}(\mathrm{F})-\sum_{k=0}^{n} \mathrm{e}\left(F_{k}\right)||=|| \mathrm{e}\left(\mathrm{F}-\sum_{k=0}^{n} F_{k}\right)||=|| \mathrm{F}-\sum_{k=0}^{n} F_{k}|| \mathrm{x} \leq \\
& || \mathrm{F}-\sum_{k=0}^{n} F_{k}|| \stackrel{n \rightarrow \infty}{\longrightarrow} 0 .
\end{aligned}
$$

Logo $\sum_{n=0}^{\infty} \alpha_{n}$ converge em E para $\alpha$.

Assim mostramos que dado $\left(\alpha_{\mathrm{n}}\right)_{\mathrm{n}} \in \mathrm{E}, \exists \alpha \in \mathrm{E}: \sum_{n=0}^{\infty}\left\|\alpha_{n}\right\|<\infty \Rightarrow \sum_{n=0}^{\infty} \alpha_{n}=\alpha$. Portanto, E é completo.

Lema 7.3.13 Todo elemento não nulo de Eé invertível em relação ao produto definido em 7.2.1, isto é, dado $\alpha \in E, \alpha \neq 0$, existe $\beta \in E$ tal que $\alpha \beta=\beta \alpha=1$, onde 1 é a unidade em $E$. 
Prova. Seja $\alpha \in \mathrm{E}, \alpha \neq 0$, pela Proposição 7.3.4, sabemos que existe $\mathrm{Y} \in \mathrm{G}_{\mathrm{X}}$ e $\mathrm{T} \in \mathrm{L}(\mathrm{Y}, \mathrm{X})$ tal que $\alpha=\mathbf{e}(\mathrm{T})$.

Afirmação: T não é estritamente singular .

De fato. Se $T$ fosse estritamente singular, teríamos $\bar{T}=\overline{0}$, onde $\overline{0}=S(Y, X)$ e assim

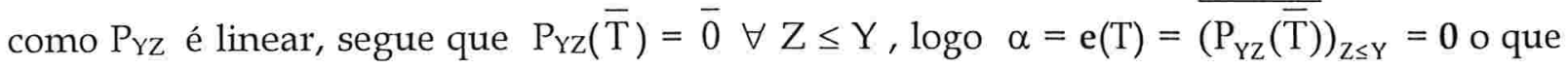
contraria nossa hipótese inicial de que $\alpha \neq 0$.

Logo por (1) segue: $\exists Y_{0}$ subespaço fechado de $Y$, com dimensão infinita, tal que $\mathrm{T}_{\mathrm{Y}_{0}}$ é isomorfismo sobre a imagem.

Agora, denotando por $\mathrm{F}=\mathrm{T}_{\mathrm{I}_{0}}$ e $\mathrm{W}=\mathrm{F}\left(\mathrm{Y}_{0}\right)$ temos que $\mathrm{F}: \mathrm{Y}_{0} \rightarrow \mathrm{W}$ é um isomorfismo. Assim para todo $Z \leq W$, podemos considerar o elemento $\beta_{Z}=P_{w Z}\left(\overline{\mathrm{F}^{-1}}\right)$.

Logo, considerando $\beta=\left(\overline{\beta_{Z}}\right) \mathrm{z} \leq \mathrm{W}$ é imediato que $\beta=\mathrm{e}\left(\mathrm{F}^{-1}\right)$, e portanto $\beta \in \mathrm{E}$.

Vamos agora mostrar que $\alpha \beta=1$.

Primeiramente, observemos que $W \in G_{X}$ é tal que $\beta_{Z} \in E_{Z Y}, \forall Z \leq W$.

$$
\beta_{Z}=P_{W Z}\left(\overline{F^{-1}}\right)=\overline{F^{-1}\left(I_{Z}+S\right)} \text { onde } I_{Z}+S: Z \rightarrow W^{\prime} \subset W \text { e } F^{-1}: W \rightarrow Y_{0} \subset Y
$$

Logo, $F^{-1}\left(I_{Z}+S\right): Z \rightarrow Y_{0} \subset Y$. Assim temos, $\beta_{Z} \in E_{Z Y}$ e $\alpha \beta={\left.\overline{\left(\alpha_{Y} \otimes \beta_{Z}\right.}\right)_{Z \leq W}}_{\text {onde: }}$

$$
\alpha_{Y} \otimes \beta_{Z}=\bar{T} \otimes \overline{F^{-1}\left(I_{Z}+S\right)}=\overline{T\left[F^{-1}\left(I_{Z}+S\right)\right]}=\overline{\left(T F^{-1}\right)\left(I_{Z}+S\right)} .
$$

e lembrando que $F=T_{\left.\right|_{y_{0}}}$, temos $T F-1=I_{W}$, assim segue que :

$$
\alpha_{\mathrm{Y}} \otimes \beta_{\mathrm{Z}}=\overline{\mathrm{I}_{\mathrm{W}}\left(\mathrm{I}_{\mathrm{Z}}+\mathrm{S}\right)}=\overline{\left(\mathrm{I}_{\mathrm{Z}}+\mathrm{S}\right)}=\overline{\mathrm{I}_{\mathrm{Z}}}, \forall \mathrm{Z} \leq \mathrm{W} .
$$

Logo, $\quad \alpha \beta={\overline{\left(\bar{I}_{z}\right)_{Z \leq W}}}_{\left.=\overline{(\bar{I}}_{Z}\right)_{Z \in G_{X}}}=1$.

Finalmente, vamos mostrar que $\beta \alpha=1$. 
Primeiramente observemos que $\beta_{\mathrm{W}}=\overline{\mathrm{F}^{-1}}, \mathrm{e}(\mathrm{T})=\alpha$, e sendo $\mathrm{F}=\mathrm{T}_{\mathrm{I}_{0}}$, segue do Lema 7.3.7

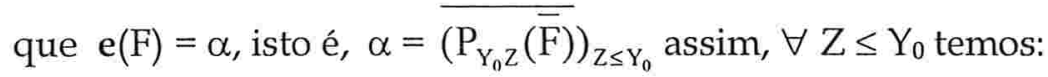

$\left.\beta_{W} \otimes \alpha_{Z}=\overline{F^{-1}} \otimes P_{Y_{0} Z}(\bar{F})=\overline{F^{-1}} \otimes \overline{F\left(I_{Z}+S\right)}=\overline{F^{-1}\left[F\left(I_{Z}+S\right)\right.}\right]=\overline{\left(F^{-1} F\right)\left(I_{Z}+S\right)}=\overline{I_{Y_{0}}\left(I_{Z}+S\right)}=\overline{I_{Z}}$.

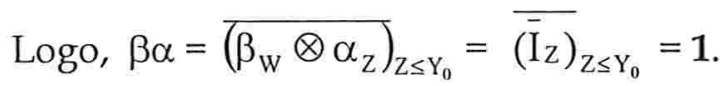

Proposição 7.3.14 Seja X um espaço de Banach complexo, hereditariamente indecomponível. Então o espaço E com a multiplicação dada na definição 7.2.1 é uma Álgebra de Banach com unidade.

Prova. Sendo X um espaço de Banach complexo, segue da própria definição, que o espaço E, dado na Definição 7.1.11, com a norma definida na Proposição 7.1.13, possui estrutura de um espaço vetorial normado e complexo. Agora, sendo E completo (ver lema 7.3.12), segue que E é um espaço de Banach complexo. Assim utilizando o resultado da Proposição 7.2.4 segue que E é uma Álgebra de Banach complexa com unidade.

Proposição 7.3.15 Seja X um espaço de Banach complexo hereditariamente indecomponível. Então o espaço E dado na definição 7.1.11 é isométrico a $\mathrm{C}$.

Prova. Pela proposição anterior vimos que E é uma Álgebra de Banach complexa com unidade, e pelo Lema 7.3.13 temos que todo elemento não nulo de E, é invertível em relação ao produto dado na definição 7.2.1, assim pelo caso complexo do Teorema de Gelfand Mazur (Teorema 2.2.3) segue que E é isométricamente isomorfo a C.

Corolário 7.3.16 Seja X um espaço de Banach complexo hereditariamente indecomponível, então E é possui dimensão 1 .

Prova. Como $\mathrm{E}=\mathrm{C}$ e $\operatorname{dim}_{\mathrm{C}} \mathrm{C}=1$ temos que $\operatorname{dim}_{\mathrm{C}} \mathrm{E}=1$.

Proposição 7.3.17 Sejam X um espaço de Banach H.I. e Y um subespaço fechado qualquer de X, com dimensão infinita. Então o espaço EYé isométrico a um subespaço de E. 
Prova. Vamos mostrar que dado $\mathrm{Y} \in \mathrm{G}_{\mathrm{x}}$, a aplicação $\theta$ dada abaixo está bem definida e é uma isometria linear.

$$
\begin{aligned}
\theta: E_{Y} & \rightarrow E \\
\alpha_{Y} & \rightarrow{\overline{\left(P_{Y Z}\left(\alpha_{Y}\right)\right)_{Z \leq Y}}} .
\end{aligned}
$$

Segue da Definição 7.3.1 e da Proposição 7.3.3 que $\theta$ está bem definida e é linear.

Vamos agora, mostrar que $\theta$ é uma isometria. De fato, segue da Observação 7.1.14 que

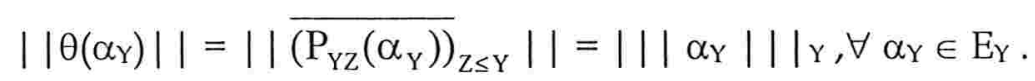

Assim, temos que $\theta$ é uma isometria linear entre EY e um subespaço de E.

Corolário 7.3.18 No caso complexo, temos $\operatorname{dim}_{\mathrm{C}}\left(E_{Y}\right)=1$.

Prova. Segue da Proposição anterior que EY é isométrico a um subespaço de E, e como no caso complexo E possui dimensão 1(ver Corolário 7.3.16), temos que $\operatorname{dim}_{\mathrm{C}}\left(E_{Y}\right)=0$ ou $\operatorname{dim}_{\mathrm{C}}\left(E_{Y}\right)=1$, mas o caso $\operatorname{dim}_{\mathrm{C}}\left(E_{Y}\right)=0$, implica $S(Y, X)=L(Y, X)$ o que é um absurdo, pois por exemplo, a inclusão de $Y$ em X não é estritamente singular. 


\section{OPERADORES EM SUBESPAÇOS DE ESPAÇOS DE BANACH HEREDITARIAMENTE INDECOMPONÍVEIS}

Neste último capítulo faremos a demonstração do principal teorema da nossa dissertação, daremos uma nova caracterização para os espaços de Banach H.I. e ainda estudaremos o que acontece quando X é um Espaço de Banach Real.

Teorema 8.1 Sejam X um espaço de Banach complexo H.I. e Y um subespaço fechado qualquer de X com dimensão infinita. Então todo operador de $Y$ em $X$ é da forma $\lambda I_{Y}+S$, onde $I_{Y}$ é a inclusão de $Y$ em $X, S$ um operador estritamente singular e $\lambda$ um número complexo.

Prova. (a) Seja $\mathrm{X}$ um Espaço de Banach Complexo, Hereditariamente Indecomponível e considere o espaço E dado na Definição 7.1.11. Pela Proposição 7.3.15, temos que E é isométrico ao espaço C dos números complexos, e assim, pelo Teorema 2.2.3 segue que a aplicação $\rho$ definida abaixo é uma isometria linear entre E e C.

$$
\begin{aligned}
\rho: E & \rightarrow C \\
\alpha & \rightarrow \lambda
\end{aligned}
$$

onde $\lambda$ é um número complexo tal que $\alpha=\lambda \mathbf{1}$, e $\mathbf{1}$ é a unidade em $\mathrm{E}$.

(b) Sejam $Y \in G_{x}$ e $T \in L(Y, X)$ arbitrários. Consideremos agora o elemento $\alpha=\mathrm{e}(\mathrm{T})$. Pela Definição 7.3.1 temos que $\alpha \in \mathrm{E}$, e assim por (a) segue que: $\exists \lambda \in \mathrm{C} / \alpha=\lambda \mathbf{1}$.

Assim, $\mathrm{e}(\mathrm{T})=\lambda 1$ implica $\mathrm{e}(\mathrm{T})-\lambda 1=0$. Agora, lembrando que é é uma aplicação linear e que $1=\mathrm{e}\left(\mathrm{I}_{Y}\right)$ temos, $\mathrm{e}(\mathrm{T})-\lambda \mathrm{e}\left(\mathrm{I}_{Y}\right)=0 \Rightarrow \mathrm{e}\left(\mathrm{T}-\lambda \mathrm{I}_{Y}\right)=0$.

Logo, segue da Observação 7.3.6 que $0=|| \mathbf{e}\left(\mathrm{T}-\lambda \mathrm{I}_{Y}\right)||=|| \mathrm{T}-\lambda \mathrm{I}_{Y}|| \mathrm{Y}$.

Assim, pela Proposição 5.3 temos que $\mathrm{T}-\lambda \mathrm{I}_{Y}$ é um operador estritamente singular, ou seja, $\mathrm{T}-\lambda \mathrm{I}_{Y}=\mathrm{S}$ onde $\mathrm{S} \in \mathrm{S}(\mathrm{Y}, \mathrm{X})$. 
Logo, $\mathrm{T}=\lambda \mathrm{I}_{Y}+\mathrm{S}$ onde $\mathrm{I}_{Y}$ é a inclusão de $\mathrm{Y}$ em $\mathrm{X}$ e $\mathrm{S}$ um operador estritamente singular.

Como $Y \in G$ x e $T \in L(Y, X)$ são arbitrários, o teorema está provado.

O próximo corolário nos fornece uma nova caracterização para os espaços de Banach complexo hereditariamente indecomponível. Observe.

Corolário 8.2 Seja X um espaço de Banach complexo de dimensão infinita. Xé H.I. se, e somente se, dado $Y \in G_{X}$, todo operador $T: Y \rightarrow X$, é da forma $\lambda I_{Y}+S$, onde $I_{Y}$ é a inclusão de $Y$ em $X, S$ um operador estritamente singular e $\lambda$ um número complexo.

Prova. Supondo que $X$ seja H.I., a demonstração é dada pelo Teorema 8.1.

Reciprocamente, suponhamos por absurdo que $X$ não seja H.I., então existe $W$ subespaço fechado de $X$, tal que $W=Y \oplus Z$, onde $Y$ e $Z$ são subespaços fechados de $X$, com dimensão infinita tais que $Y \cap Z=\{0\}$.

Considere agora a projeção canônica de $Y \oplus Z$ em $Y$, isto é:

$$
\begin{aligned}
P: W & \rightarrow Y \\
w & \rightarrow P(w)=P(y+z)=y
\end{aligned}
$$

Por hipótese temos que $\mathrm{P}=\lambda \mathrm{I}_{\mathrm{W}}+\mathrm{S}$, onde $\mathrm{I}_{\mathrm{W}}$ é a inclusão de $\mathrm{W}$ em $\mathrm{X}, \mathrm{S}$ um operador estritamente singular e $\lambda$ um número complexo. Assim, pela Proposição 3.3.5 segue que $\operatorname{dim}(\operatorname{Ker} P)<\infty$, o que é um absurdo, pois dado $\forall z \in Z$, temos que $P(z)=0$, ou seja, ker $\mathrm{P}=\mathrm{Z}$ e portando $\operatorname{dim}(\operatorname{Ker} \mathrm{P})=\infty$.

Logo, Xé hereditariamente indecomponível.

Observação 8.3 A hipótese de X ser um espaço vetorial complexo e não real, foi essencial para assim, podermos utilizar o caso complexo do Teorema de Gelfand - Mazur (Teorema 2.2.3), contudo observem que sendo X um espaço de Banach H.I., real ou complexo, segue da Proposição 7.2.4 e do Lema 7.3.13 que o espaço E construído anteriormente possui uma estrutura de álgebra normada com unidade, onde todo elemento não nulo é invertivel em relação ao produto definido em E, ou seja, E possui estrutura de álgebra normada com divisão. 
Observação 8.4 Vimos que no caso complexo, $\operatorname{dim}_{\mathrm{C}}\left(E_{Y}\right)=1$, (ver Corolário 7.3.18).

O último resultado deste trabalho foi obtido por Ferenczi em [4], onde é provado que no caso real o espaço EY possui dimensão menor ou igual a 4 .

Teorema 8.5 Seja X um espaço de Banach real H.I. Então, para todo $Y \in G_{X}$, temos que $\operatorname{dim}\left(E_{Y}\right) \leq 4$.

Prova. Decorre da Observação 8.3 que o espaço E, dado na Definição 7.1.11, possui estrutura de uma álgebra normada real, com divisão, assim segue do caso real do Teorema de Gelfand - Mazur (Teorema 2.3.15), que $\mathrm{E}$ é isomorfo a $\mathbf{R}, \mathrm{C}$ ou $\mathbf{H}$, onde $\mathbf{H}$ denota a álgebra dos quatérnios. Agora como Ey é isomorfo a um subespaço de E (ver Proposição 7.3.17), segue então que a dimensão do espaço Ey será 1,2, 3 ou 4.

Finalmente destacamos que ainda permanece em aberto o seguinte problema:

"Sendo X um espaço de Banach real H.I. e $Y \in G_{X}$, qual será a dimensão do espaço EY?"

Observações 8.6 A seguir faremos algumas considerações sobre o problema em aberto.

i) Vale destacar que o espaço $X_{G M}$ construído por Gotvers e Maurey (ver [6]), no caso real, satisfaz $\operatorname{dim} E_{Y}=1, \forall Y$, e que também $\operatorname{dim} E_{Y}=1, \forall Y$, implica que $X_{G M}$ hereditariamente indecomponível, (prova do Corolário 8.2 também válido para o caso real).

ii) Segue dos resultados de "Hereditarily finitely decomposable Banach spaces"(ver [4]), que se $X=X_{G M} \oplus X_{G M}$, temos $\operatorname{dim} E_{Y} \leq 4, \forall Y$. Mas, X não é H.I.

iii) EY é isométrico uma subespaço vetorial de E, mas não necessariamente é uma sub-álgebra de E, pois não podemos necessariamente compor $T \in L(Y, X) \operatorname{com} U \in L(Y, X)$. A exceção é quando $E_{X}=L(X) / S(X)$, pois $E_{X}$ tem estrutura de álgebra, e $\theta: E_{X} \rightarrow E$ é um isomorfismo de álgebras sobre a imagem, portanto $E_{X}$ é uma sub-álgebra normada com divisão de $E$, isométrica a $\mathrm{R}, \mathrm{C}$ ou $\mathrm{H}$. Então

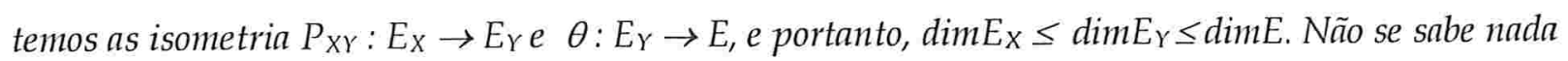
sobre o que pode acontecer a partir disso. DimE $E_{Y}=3$ é possivel? $\operatorname{dim} E_{X}<\operatorname{dim} E_{Y}<\operatorname{dim} E$ é possível?

iv) De fato, parece ser possível construir uma versão real do espaço H.I. de Govvers e Maurey, onde $\operatorname{dim} E_{X}=2$ e provavelmente $\operatorname{dim} E_{Y}=2$, mas até o momento isso nunca foi escrito. 


\section{REFERÊNCIAS BIBLIOGRÁFICAS}

[1] Cristina, C., O primeiro espaço de Banach hereditariamente indecomponível. IME-USP, 2000 (Dissertação de Mestrado).

[2] Bonsall F.F. e Duncan J. , Complete Normed Agebras. Springer - Verlag, New York, 1973.

[3] Ferenczi, V. , Operators on subspaces of hereditarily indecomposable Banach spaces. Bull. London Math. Soc. 29, (1996), 338 - 344.

[4] Ferenczi, V., Hereditarily finitely decomposable Banach spaces. Studia Mathematica 123 (2) (1997), $135-149$.

[5] Galego, E.M., Alguns aspectos da teoria geométrica de espaços de Banach através de $c_{0}(N)$. 43o Seminário Brasileiro de Análise, IME-USP, 1996.

[6] Gowers, W. T. e Maurey, B., The unconditional basic sequence problem. J. Amer. Math. Soc. 6 (1993), $851-874$.

[7] Hönig, C.S., Análise Funcional e Aplicações. V.1, IME-USP, 1970.

[8] Hönig, C.S., Análise Funcional e Aplicações. V.2, IME-USP, 1970.

[9] Lima, E.L., Espaços Métricos. IMPA. Rio de Janeiro, 1993.

[10] Lindenstrauss, J.e Tzafriri, L.,Classical Banach Spaces, V.1, New York, Springer, 1977.

[11] Taylor, A.E., Introduction to Functional Analysis. Wiley \& Sons, New York, 1958.

[12] Vieira, D.M.S., Operadores de Composição entre as Álgebras Clássicas de Funções Analíticas. IME-USP, 2000 (Dissertação de Mestrado). 